\title{
External Quality-Assurance Results for the National Atmospheric Deposition Program/ National Trends Network, 1997-99
}

Water-Resources Investigations Report 03-4027

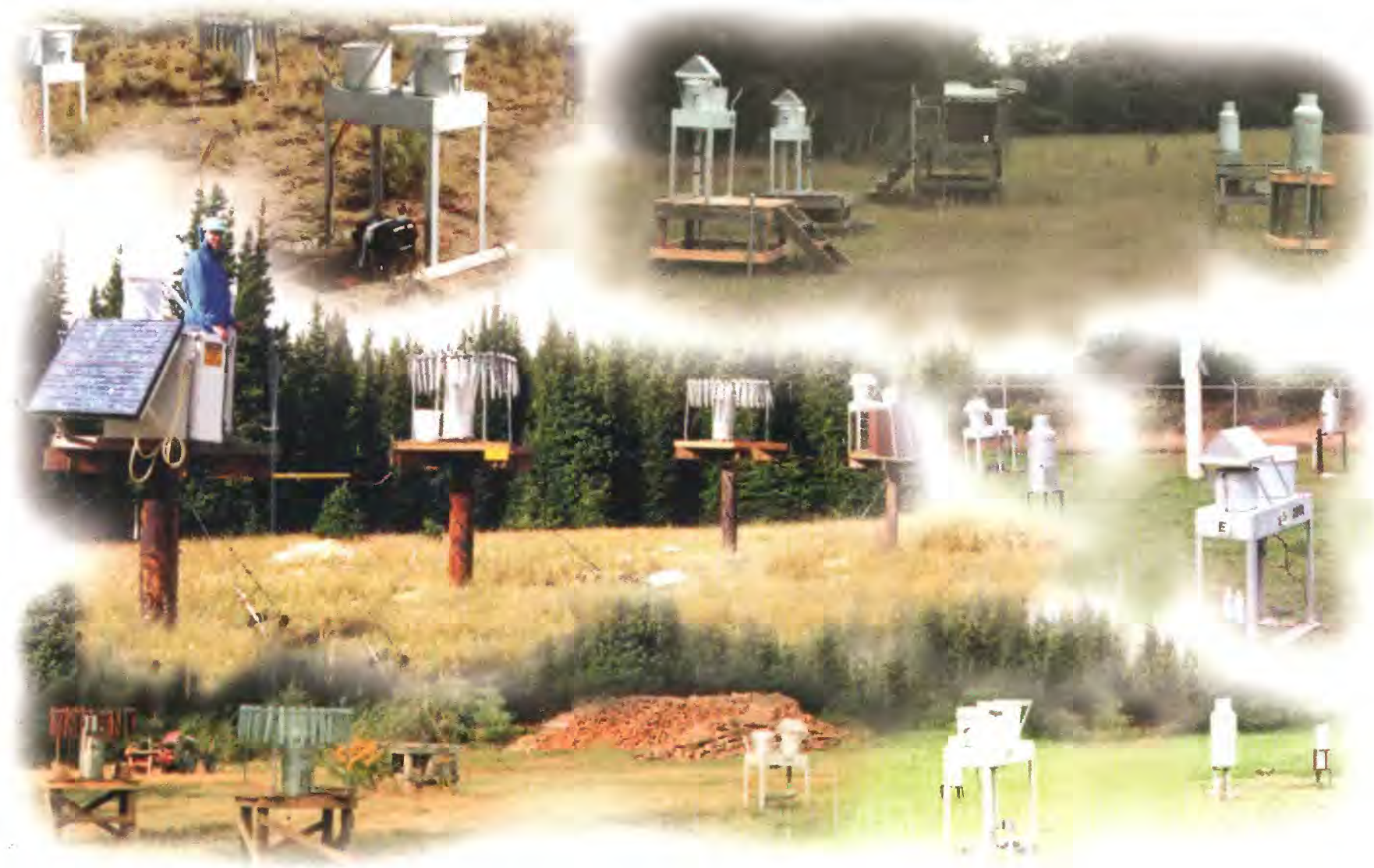

U.S. Department of the Interior

U.S. Geological Survey 
Much appreciation is extended to the following U.S. Geological employees for their contributions to this report:

Members of the Colorado District Publishing Section (Alene Brogan, Joy Monson, Robert Olmstead, and John Evans) for final production of the report; and Mary Kidd for her editorial and verifications reviews.

Appreciation also is extended to the technical reviewers:

Dr. Tom Butler of Cornell University and Jane Rothert of the Illinois State Water Survey.

Front cover photographs are of the National Atmospheric Deposition Program/ National Trends Network Sites used in the collocated sampler program, 1997-99 (clockwise from top left):

Silver Lake Ranger Station in Oregon (site OR09/09OR)

Cedar Creek in Minnesota (site MN01/01MN)

Shenandoah National Park in Virginia (site VA28/28VA)

Quincy, Florida (site FL14/14FL)

Quabbin Reservoir in Massachusetts (site MA08/08/MA)

Brooklyn Lake in Wyoming (site WY95/95WY)

Back cover photographs:

Belfort 5-780 rain gage and calibration weights (left photograph) and Aerochem Metrics wet-deposition collector at Brooklyn Lake site in Wyoming. 


\section{External Quality-Assurance Results for the National Atmospheric Deposition Program/National Trends Network, 1997-99}

By John D. Gordon, Natalie E. Latysh, and Sandy J. Lindholm

U.S. GEOLOGICAL SURVEY

Water-Resources Investigations Report 03-4027 


\title{
U.S. DEPARTMENT OF THE INTERIOR GALE A. NORTON, Secretary
}

\author{
U.S. GEOLOGICAL SURVEY
}

Charles G. Groat, Director

The use of firm, trade, and brand names in this report is for identification purposes only and does not constitute endorsement by the U.S. Geological Survey.

For additional information write to:

Chief, Branch of Quality Systems

U.S. Geological Survey

Box 25046, Mail Stop 401

Denver Federal Center

Denver, CO 80225-0046
Copies of this report can be purchased from:

U.S. Geological Survey

Information Services

Box 25286

Denver Federal Center

Denver, CO 80225 


\section{CONTENTS}

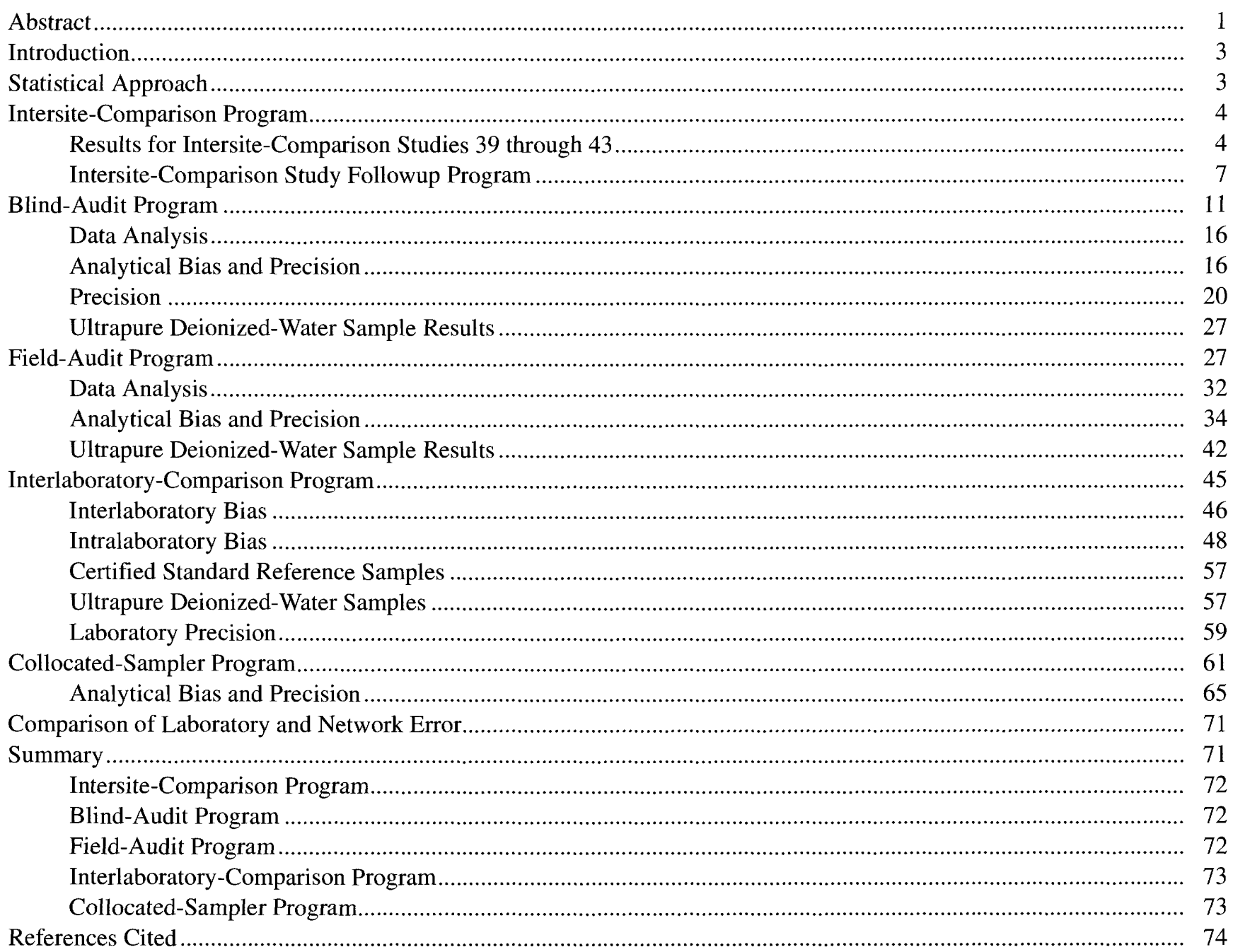

\section{FIGURES}

1. Flowchart showing intersite-comparison program of the U.S. Geological Survey.

2-6. Graphs showing:

2. Distribution of $\mathrm{pH}$ and specific-conductance values for intersite-comparison studies 39 and 40 , completed in 1997

3. Distribution of $\mathrm{pH}$ and specific-conductance values for intersite-comparison studies 41 and 42 , completed in 1998

4. Distribution of $\mathrm{pH}$ and specific-conductance values for intersite-comparison study 43, completed in 1999

5. Percentiles for $\mathrm{pH}$ and specific conductance from intersite-comparison studies 39 through $43 \ldots \ldots \ldots \ldots \ldots \ldots \ldots . . .11$

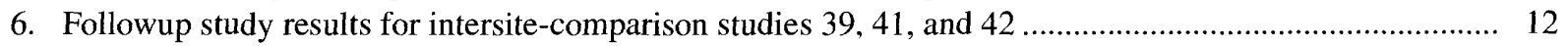

7. Flowchart showing blind-audit program of the U.S. Geological Survey ......................................................... 15

8-12. Boxplots showing:

8. Paired bucket-sample concentrations minus bottle-sample concentrations in the blind-audit program during 1997 through 1999.

9. Relation between paired blind-audit differences for major ions and the analyte concentrations of solutions used in the blind-audit program during 1997 through 1999. 
10. Relation between paired blind-audit differences for hydrogen ion and specific conductance and the analyte concentrations of solutions used in the blind-audit program during 1997 through 1999

11. Relation between paired blind-audit differences for the major ions and sample volume for the 250-, 500-, and 1,000-milliliter U.S. Geological Survey solution samples during 1997 through 1999

12. Relation between paired blind-audit differences for hydrogen ion and specific conductance and sample volume for the 250-, 500-, and 1,000-milliliter U.S. Geological Survey solution samples during 1997 and 1999

13-16. Graphs showing:

13. Locally weighted scatterplot smoothing for the blind-audit program for the analytes sulfate and chloride during 1997 through 1999.

14. Locally weighted scatterplot smoothing for the blind-audit program for the analytes ammonium and potassium during 1997 through 1999

15. Locally weighted scatterplot smoothing for the blind-audit program for the analytes nitrate and magnesium during 1997 through 1999

16. Locally weighted scatterplot smoothing for the blind-audit program for the analytes calcium and sodium during 1997 through 1999

17. Flowchart showing field-audit program of the U.S. Geological Survey.....

18-22. Boxplots showing:

18. Paired bucket-sample concentrations minus bottle-sample concentrations in the field-audit program during 1997 through 1999

19. Relation between paired field-audit differences for major ions and the analyte concentrations of solutions used in the field-audit program during 1997 through 1999

20. Relation between paired field-audit differences for hydrogen ion and specific conductance and the analyte concentrations of solutions used in the field-audit program during 1997 through 1999.

21. Relation between paired field-audit differences for the major ions and sample volume for the 250-, 500-, and 1,000-milliliter U.S. Geological Survey solution samples during 1997 through 1999.

22. Relation between paired field-audit differences for hydrogen ion and specific conductance and sample volume for 250-, 500-, and 1,000-milliliter U.S. Geological Survey solution samples during 1997 through 1999

23. Flowchart showing interlaboratory-comparison program of the U.S. Geological Survey......

24-30. Graphs showing:

24. Difference between the measured value reported by the Acid Deposition and Oxidant Research Center and the median value calculated for all participating laboratories in the interlaboratorycomparison program during 1997 through 1999.

25. Difference between the measured value reported by the Illinois State Water Survey, Central Analytical Laboratory and the median value calculated for all participating laboratories in the interlaboratorycomparison program during 1997 through 1999.

26. Difference between the measured value reported by the Environmental Science and

Engineering, Inc., Laboratory and the median value calculated for all participating laboratories in the interlaboratory-comparison program during 1997 through 1999.

27. Difference between the measured value reported by the Ontario Ministry of the Environment, Dorset Research Facility in Ontario, Canada, and the median value calculated for all participating laboratories in the interlaboratory-comparison program during 1997 through 1999.

28. Difference between the measured value reported by the Meteorological Services of Canada and the median value calculated for all participating laboratories in the interlaboratory-comparison program during 1997 through 1999

29. Difference between the measured value reported by the Norwegian Institute for Air Research and the median value calculated for all participating laboratories in the interlaboratorycomparison program during 1997 through 1999.

30. Difference between the measured value reported by the Shepard Analytical Services and the median value calculated for all participating laboratories in the interlaboratory-comparison program during 1997 through 1999 
31. Graphs depicting the number of data points outside the control limits for the lllinois State Water Survey,

Central Analytical Laboratory during 1997 through 1999

32. Map showing location of National Atmospheric Deposition Program/National Trends Network sites with collocated samplers in water years 1997 through 1999.

33-37. Graphs showing:

33. Median concentrations for selected analytes in weekly samples collected at six National

Atmospheric Deposition Program/National Trends Network sites with collocated samplers during 1997 through 1999

34. Median hydrogen-ion concentration, specific conductance, sample volume, and precipitation depth at National Atmospheric Deposition Program/National Trends Network sites with collocated samplers during 1997 through 1999

35. Bias for analyte concentration, deposition, and other physical parameters for weekly samples from collocated wet-dry precipitation collectors and precipitation depth from collocated rain gages during 1997 through 1999.

36. Median absolute difference for analyte concentration and deposition for weekly samples from collocated precipitation collectors during 1997 through 1999 for selected analytes.

37. Median absolute difference for hydrogen ion, specific conductance, sample volume, and precipitation depth for weekly samples from collocated precipitation collectors and precipitation depth from collocated rain gages during 1997 through 1999.

\section{TABLES}

1. Site-operator responses and summary statistics for the 1997 intersite-comparison program.

2. Site-operator responses and summary statistics for the 1998-99 intersite-comparison program.........

3. Solutions used in the 1997-99 blind-audit, field-audit, and interlaboratory-comparison programs

4. Target values for solutions used in the 1997-99 blind-audit program, field-audit program, and interlaboratory-comparison program

5. Selected statistics for the paired bucket-sample concentration minus bottle-sample concentration differences in the blind-audit program during 1997 through 1999

6. Results of the tests for bias in the blind-audit program during 1997 through 1999 , using the paired t-test and a Wilcoxon signed-rank test

7. Results of the Kruskal-Wallis analysis of variance test to determine the relation between paired blindaudit sample differences and the target concentrations used in the blind-audit program in 1997, 1998, and 1999

8. Relative and absolute bucket minus bottle differences calculated as a percentage of the target bottle

concentration for each analyte during 1997 through 1999 for the blind-audit program.

9. Results of the 1997 through 1999 Kruskal-Wallis analysis of variance tests to determine if bucket minus bottle differences for the 250-, 500-, and 1,000-milliliter samples of the USGS solution used in the blind-audit program have equivalent distributions.

10. Number of determinations exceeding the minimum reporting limit for the Ultrapure deionized-water samples submitted annually as part of the blind-audit program, 1997, 1998, and 1999.

11. Results of the tests for bias in the field-audit program during 1997 through 1999, using the paired t-test and a Wilcoxon signed-rank test on samples where rinse water was present.

12. Results of the tests for bias in the field-audit program during 1997 through 1999, using the paired t-test and a Wilcoxon signed-rank test on samples where rinse water was not present.

13. Selected statistics for the paired bucket-sample concentration minus bottle-sample concentration differences in the field-audit program during 1997 through 1999 for "wet" and "dry" coded samples...

14. Results of the Kruskal-Wallis analysis of variance test to determine the relation between paired fieldaudit sample differences and the target concentrations used in the field-audit program in 1997, 1998, and 1999

15. Relative and absolute bucket minus bottle differences calculated as a percentage of the target concentration for the bottle portion for each analyte during 1997 through 1999 for the field-audit program 
16. Results of the 1997 through 1999 Kruskal-Wallis analysis of variance tests to determine if bucket minus bottle differences for the 250-, 500-, and 1,000-milliliter samples of the USGS solution used in the field-audit program have equivalent distributions

17. Number of determinations exceeding the minimum reporting limit for the 11 Ultrapure deionizedwater samples submitted in 1997, the 22 samples submitted in 1998, and the 24 submitted in 1999 as part of the field-audit program.

18. Summary of laboratory median values per analyte range reported by reference sample supplier High Purity Standards (HPS) for standard reference materials SP-97 and SP-98 during 1997 through 1999

19. Number of analyte determinations greater than the largest minimum reporting limit used by any participating laboratory for each ion for the Ultrapure deionized-water samples during 1997 through 1999

20. Fiftieth and 90th percentile absolute differences for analysis of replicate samples determined by seven laboratories participating in the 1997 through 1999 interlaboratory-comparison program

21. Median relative error for analyte concentrations and physical parameters from weekly collocated precipitation samples and replicate samples in 1997, 1998, and 1999

CONVERSION FACTORS, TERMS, AND ABBREVIATIONS

\begin{tabular}{rll}
\hline Multiply & \multicolumn{1}{c}{ By } & To obtain \\
\hline centimeter $(\mathrm{cm})$ & 0.3937 & inch \\
liter $(\mathrm{L})$ & 1.057 & quart \\
milliliter $(\mathrm{mL})$ & 0.03381 & ounce, fluid
\end{tabular}

Temperature can be converted from degree Celsius $\left({ }^{\circ} \mathrm{C}\right)$ to degree Fahrenheit $\left({ }^{\circ} \mathrm{F}\right)$ by using the following equation:

$$
{ }^{\circ} \mathrm{F}=9 / 5\left({ }^{\circ} \mathrm{C}\right)+32
$$

\section{The following terms and abbreviations also are used in this report:}

microsiemens per centimeter at 25 degrees Celsius $(\mu \mathrm{S} / \mathrm{cm})$

megohm (M $\Omega)$

microequivalents per liter $(\mu \mathrm{eq} / \mathrm{L})$

milligrams per liter $(\mathrm{mg} / \mathrm{L})$

kilograms per hectare $(\mathrm{kg} / \mathrm{ha})$

microgram $(\mu \mathrm{g})$

absolute value of $x=|x|$, where $x$ takes the form of numerical values or algebraic expressions

maximum probability of rejecting the null hypothesis when it is true $(\alpha)$ ) 


\title{
External Quality-Assurance Results for the National Atmospheric Deposition Program/National Trends Network, 1997-99
}

\author{
By John D. Gordon, Natalie E. Latysh, and Sandy J. Lindholm
}

\begin{abstract}
Five external quality-assurance programs were operated by the U.S. Geological Survey for the National Atmospheric Deposition Program/ National Trends Network (NADP/NTN) during 1997 through 1999: the intersite-comparison program, the blind-audit program, the fieldaudit program, the interlaboratory-comparison program, and the collocated-sampler program. The intersite-comparison program assesses the accuracy of $\mathrm{pH}$ and specific-conductance determinations made by NADP/NTN site operators. In two 1997 intersite-comparison studies, 83.7 and 85.8 percent of the $\mathrm{pH}$ determinations met the NADP/NTN accuracy goals, whereas 97.3 and 92.4 percent of the specific-conductance determinations met the NADP/NTN accuracy goals. The percentage of $\mathrm{pH}$ and specific-conductance determinations that met the accuracy goals in 1998 were, for the most part, higher than in 1997. In two 1998 studies, 90.9 and 90.3 percent of the $\mathrm{pH}$ determinations met the accuracy goals compared to 94.7 and 96.0 percent of the specificconductance measurements meeting the accuracy goals. In one 1999 intersite-comparison study, 89.5 percent and 99.4 percent of $\mathrm{pH}$ and specificconductance determinations, respectively, met the NADP/NTN accuracy goals.

The blind-audit program evaluates the effects of routine sample handling, processing, and shipping on the analytical bias and precision of weekly precipitation samples. A portion of
\end{abstract}

the blind-audit sample subject to the normal onsite handling and processing of a weekly precipitation sample is referred to as the bucket portion, whereas the portion receiving only minimal handling is referred to as the bottle portion. Positive bias in regard to blind-audit results indicates that the bucket portion has a higher concentration than the bottle portion. The paired t-test for the 1997 through 1999 blindaudit data indicates that routine sample handling, processing, and shipping introduced a positive bias $(\alpha=0.05)$ for calcium and chloride and a negative bias $(\alpha=0.05)$ for hydrogen ion. During 1997 through 1999, the median paired differences between the bucket and bottle portions ranged from 0.00 milligram per liter for nitrate and ammonium to +0.010 milligram per liter for both chloride and sulfate. The median paired difference between the bucket and bottle portions for hydrogen ion was -1.086 microequivalents per liter, whereas for specific conductance, the median paired difference between the bucket and bottle portions was -0.200 microsiemen per centimeter during 1997 through 1999.

Surface-chemistry effects due to variable amounts of precipitation contacting prewashed sample-collection and shipping-container surfaces were studied in the blind-audit program by using three different sample volumes. The samplecollection and shipping containers used for the blind-audit study were obtained from the site operator's supply and could have been used for precipitation samples. Results of a Kruskal-Wallis 
analysis of variance test of the relation between paired blind-audit sample differences in units of concentration and sample volume were statistically significant for magnesium, chloride, sulfate, and hydrogen ion during 1997 through 1999. Before 1994, at least 5 of the 10 analytes displayed a statistically significant difference between paired blind-audit differences in units of concentration and sample volume, supporting the premise that chemical reactions between the 13-liter bucket shipping container (primarily the butadiene o-ring lid of the shipping container) and the sample, which resulted in an increasing loss of hydrogen ion with increasing volume, have been eliminated by the new 1-liter bottle sampleshipping protocol.

The field-audit program measures the effects of field exposure, handling, and processing on the chemistry of NADP/NTN precipitation samples. In the field-audit program, the site operator is instructed to process and submit a qualitycontrol sample following a standard 7-day, Tuesday-to-Tuesday sampling period with no precipitation. The Wilcoxon signed-rank test indicated that no bias was introduced during routine sample handling, processing, and shipping for any of the analytes during 1997 through 1999. However, the paired t-test indicated statistically significant differences $(\alpha=0.05)$ for all of the analytes except for sulfate and specific conductance for the "wet" coded samples and all but sodium and specific conductance for the "dry" coded samples during the same time period.

Similar to the blind-audit program, surface-chemistry effects due to variable amounts of precipitation contacting prewashed samplecollection and shipping container surfaces also were studied in the field-audit program by using three different sample volumes. Results of a Kruskal-Wallis analysis of variance test of the relation between paired field-audit sample differences in units of concentration and sample volume were statistically significant for magnesium, chloride, and sulfate during 1997 through 1999. These were the same analytes that were statistically significant in the blind-audit program during the same time period. The blind-audit program also showed a statistically significant difference for hydrogen ion that the field-audit program did not show.

The two objectives of the interlaboratorycomparison program are: (1) to determine if statistically significant differences exist among the analytical results of participating laboratories, and (2) to estimate the analytical precision of participating laboratories. In 1997, results of the Friedman test indicated significant $(\alpha=0.05)$ differences in analyte measurements among the five participating laboratories for all analytes except potassium. In 1998, the Friedman test indicated significant $(\alpha=0.05)$ differences in analyte measurements among the five participating laboratories for all 10 parameters. The Friedman test indicated there were statistically significant $(\alpha=0.05)$ differences in the results from the seven participating laboratories for all analytes in 1999.

Bar graphs depicting the number of data points outside the control limits were prepared for the Central Analytical Laboratory in 1997, 1998, and 1999. For magnesium, potassium, chloride, $\mathrm{pH}$, and specific conductance, the number of data points outside the control limits decreased from 1997 to 1999. Sodium and ammonium had an upward trend in the number of data points outside the control limits during the same time period.

Intralaboratory bias was indicated for most laboratories in tests of certified analyte concentrations from standard reference material samples and from Ultrapure deionized-water samples. Precision estimates for the cations at the 50th percentile exhibited less variability than precision estimates at the 50th percentile for the anions, $\mathrm{pH}$, and specific conductance for the seven laboratories that participated in the interlaboratory-comparison program between 1997 and 1999.

In October 1988, the collocated-sampler program was established to provide a method of estimating the overall bias and precision of the precipitation-monitoring system used by the NADP/NTN. Results from the collocated-sampler 
program indicated that the median relative error, calculated from deposition amounts, exceeded 20 percent for potassium at all but two of the 1997-99 collocated sites. The median relative error, calculated from concentration amounts, was less than 10 percent for sulfate, specific conductance, and sample volume for all of the 1997-99 collocated sites. The sites with the lowest median hydrogen-ion concentrations had much higher median relative errors associated with their paired-sample analyses. The median relative errors for hydrogen-ion concentration were less than 10 percent at three sites, and these same sites had median hydrogen-ion concentrations of $18 \mu \mathrm{eq} / \mathrm{L}$ or greater. For sites with median hydrogen-ion concentrations less than $10 \mu \mathrm{eq} / \mathrm{L}$, the median relative errors exceeded 12 percent.

\section{INTRODUCTION}

A fundamental objective of the National Atmospheric Deposition Program (NADP) is to provide scientific investigators worldwide with a longterm, high-quality database of atmospheric-deposition information (Nilles, 2001). The National Atmospheric Deposition Program/National Trends Network (NADP/NTN) has investigated the occurrence and effects of wet deposition across the United States since 1978 (Robertson and Wilson, 1985; Peden, 1986). Research scientists use NADP/NTN data to study the effects of acidic deposition on human health and the environment. All operators of NADP/NTN sites adhere to the same sample collection and analysis methods. Identical wet-deposition collectors, described by Bigelow and Dossett (1988), and standard NADP/NTN sample-handling and shipping protocols are followed at the sites. Samples from the NADP/NTN sites are sent to the Illinois State Water Survey, Central Analytical Laboratory (CAL) for analysis.

This report describes the results of the external quality-assurance (QA) programs operated by the U.S. Geological Survey (USGS) in support of the NADP/NTN during 1997 through 1999. These programs are designed to (1) assess the accuracy of onsite determinations of $\mathrm{pH}$ and specific conductance (intersitecomparison program); (2) evaluate the effects of routine handling, processing, and shipping of wet-deposition samples collected within the NADP/NTN on bias and precision (blind-audit program); (3) evaluate the effects of field exposure of the sample-collection equipment, handling, and processing on precipitation chemistry (field-audit program); (4) estimate the bias and precision of analytical results determined by separate laboratories routinely measuring wet deposition (interlaboratorycomparison program); and (5) estimate the overall bias and precision of the monitoring system, from the point of sample collection through storage of data in the NADP/NTN database (collocated-sampler program). A protocol report providing detailed information on the QA procedures and analytical methods is available (See and others, 1990). Results for the constituents measured by the NADP/NTN (with the exception of orthophosphate) are included in this report. The term "major ions," in this text, refers to calcium, magnesium, sodium, potassium, ammonium, chloride, nitrate, and sulfate. Results are presented in the following systematic manner: throughout this report, results for cations, in alphabetical order (calcium, magnesium, sodium, and potassium) are described first, followed by the results for anions, in alphabetical order (ammonium, chloride, nitrate, and sulfate), followed, where appropriate, by the results for hydrogen ion and specific conductance.

\section{STATISTICAL APPROACH}

Rank-based alternatives to traditional hypothesis testing compose the statistical analysis framework in this report. Nonparametric statistical tests were used in this report because the data sets do not adhere completely to the normal distribution requirements of traditional statistics. Hypothesis tests included the Wilcoxon signed-rank test, the Kruskal-Wallis test, and the Friedman test. The Wilcoxon signed-rank test determines if there is a shift in the distribution location due to the treatment of a paired replicate analysis (Hollander and Wolfe, 1999). Because the Wilcoxon signed-rank test is slightly less powerful for paired samples than the paired t-test (Kanji, 1993), results from the paired $t$-test were included for some of the paired analyses for comparison purposes. The Kruskal-Wallis test (Iman and Conover, 1983) was used to compare two or more independent samples (SAS Institute Inc., 1989). The Friedman test was used to investigate the significance of the difference in response to two or more treatments applied to any number of subjects (Kanji, 1993). 
The hypothesis tests were based on two-sided rather than one-sided alternatives. Huntsberger and Billingsley (1981) provide a detailed explanation of two-sided and one-sided hypothesis testing. Concise graphical displays such as boxplots were used to depict data distributions and provide visual representations of NADP/NTN data quality. Tukey's "schematic plot" version of the boxplot (Chambers and others, 1983) was used for all boxplots. Values outside the whiskers are graphed individually as an asterisk (Helsel and Hirsch, 1992). The magnitude of measurement bias was quantified in several ways for the convenience of the reader, including units of concentration, mass, and percent differences.

\section{INTERSITE-COMPARISON PROGRAM}

NADP/NTN site operators measure $\mathrm{pH}$ and specific conductance on weekly precipitation wetdeposition samples, provided sample volume considerations are satisfied. Due to the low ionic strength of precipitation, minor changes in precipitation chemistry may occur between sample collection and laboratory analysis (Bigelow and others, 1989). Many authors have determined that onsite measurements (of $\mathrm{pH}$ in particular) are more representative of precipitation than subsequent laboratory determinations (Hem, 1985). Intersite-comparison studies are completed by the USGS to assess onsite measurement precision and accuracy. If measurements are not accurate, site operators are provided troubleshooting assistance by the USGS.

In the intersite-comparison studies, site operators measure the $\mathrm{pH}$ and specific conductance of synthetic precipitation-check samples (reference solutions) prepared by the USGS, and the accuracy of these measurements is compared with the target value of reference solutions. Protocols identical to NADP/NTN weekly sample measurement methods are used (Gordon and others, 1991; Bigelow and Dossett, 1988). The reference solutions have $\mathrm{pH}$ and specific conductance similar to natural wet-deposition samples collected by the NADP/NTN. Reference solutions are prepared by adding nitric acid to deionized water. The $\mathrm{pH}$ is adjusted to a specific value from 3.9 to 5.3. Specific conductance of the solution is a function of the nitric acid and any salts that are added to increase conductivity. The target values of the solutions are validated through nitrate analysis and $\mathrm{pH}$ and specificconductance measurements.
Accuracy goals account for the increased difficulty of measuring $\mathrm{pH}$ as the hydrogen-ion concentration decreases (Gordon, 1999). Accuracy goals for $\mathrm{pH}$ measurements are based on a multiple-regression function that incorporates the solution's hydrogenion concentration and the results from past intersite studies. The accuracy goals are symmetrical in units of hydrogen ion and, therefore, are asymmetrical in units of $\mathrm{pH}$. The specific-conductance values for all of the intersite comparison solutions used during 1997 through 1999 were between 10 and $60 \mu \mathrm{S} / \mathrm{cm}$. For specific conductance, if the most probable specific conductance was greater than $10 \mu \mathrm{S} / \mathrm{cm}$ but less than or equal to $60 \mu \mathrm{S} / \mathrm{cm}$, the accuracy criterion was $\pm 4 \mu \mathrm{S} / \mathrm{cm}$.

As in past years (Gordon, 1999; See and others, 1989), the median site-operator values were used as the most probable values for intersite solutions used during 1997 through 1999. The median values from approximately 200 site-operator measurements are considered a more accurate representation of the most likely values for the intersite solutions than either a few in-house measurements or the theoretical values. Previous studies have found no appreciable deterioration of intersite solutions over the duration of the studies, which further supports the use of the median site-operator values as the most probable values (Gordon and others, 1995).

\section{Results for Intersite-Comparison Studies 39 through 43}

A flowchart depicting the chronological order of the intersite-comparison program is shown in figure 1 . Intersite studies 39 and 40 were completed in June and December 1997, respectively; studies 41 and 42 were completed in June and December 1998, respectively; and study 43 was completed in June 1999. From the day the samples were mailed by the USGS to the site operators, operators were allowed 45 days to perform the field measurements. Sites were not included in the data analysis used to determine the percentage of sites achieving goals if (1) responses were received after the closing date of the study, (2) the field equipment was completely inoperable, (3) the site was not in operation at the time of the study, or (4) the site did not perform field chemistry. Table 1 contains a summary of the results for studies 39 and 40 . Table 2 summarizes the results for studies 41,42 , and 43 . The accuracy goals for each study also are provided in tables 1 and 2 . 


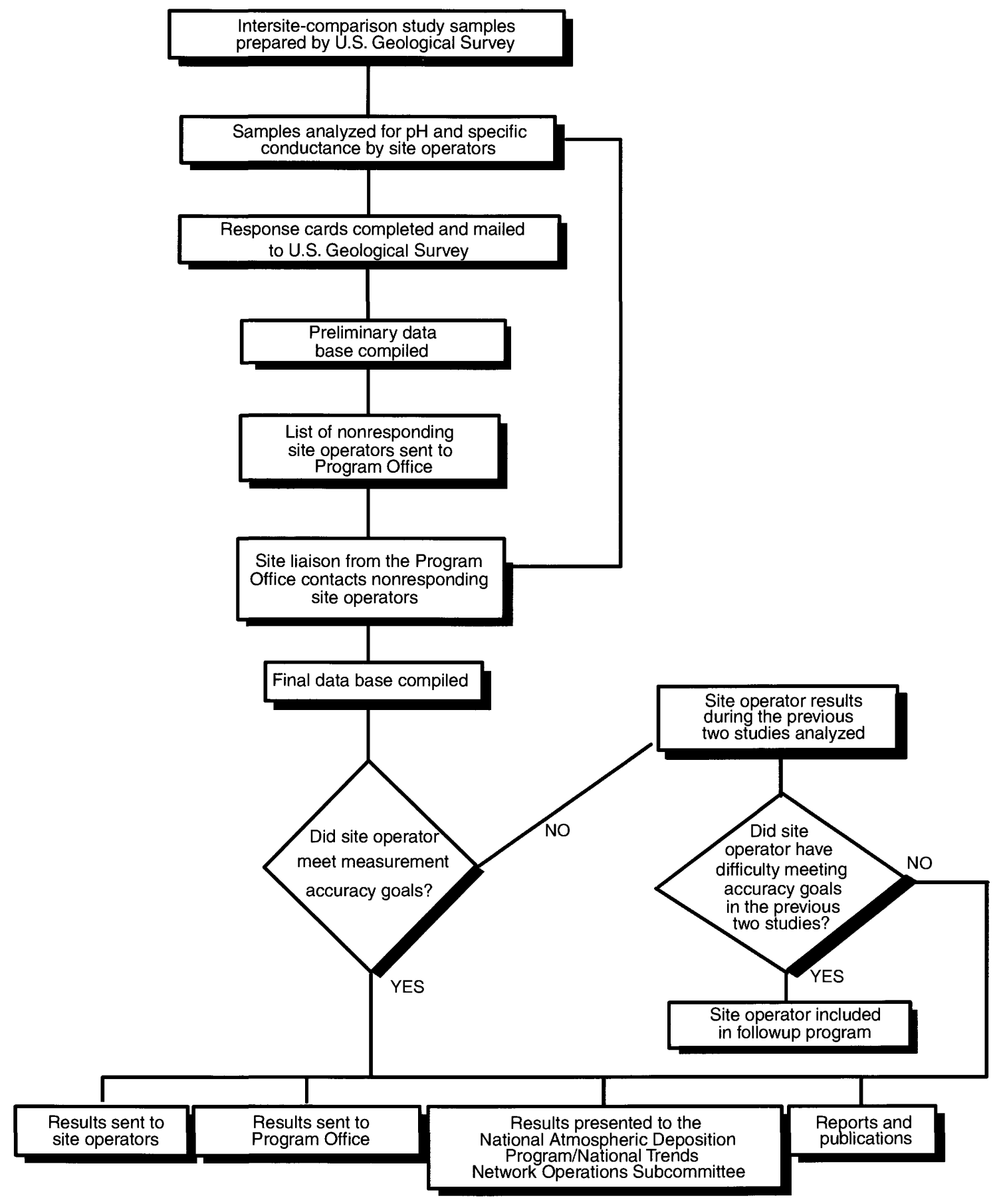

Figure 1. Intersite-comparison program of the U.S. Geological Survey.

The reference solution target $\mathrm{pH}$ for intersitecomparison study 39 was 4.75 ; the resulting median $\mathrm{pH}$ of site operators' responses was 4.78 . The target specific conductance of study 39 was increased, by the addition of potassium chloride, from $7.5 \mu \mathrm{S} / \mathrm{cm}$ to $13.0 \mu \mathrm{S} / \mathrm{cm}$; the median value determined from all site-operator measurements was $13.0 \mu \mathrm{S} / \mathrm{cm}$. More than 83 percent ( 154 out of 184 ) of the site operators met the $\mathrm{pH}$ accuracy goal of 4.62 to 4.93 in intersitecomparison study 39 . The NADP/NTN accuracy goals for specific conductance for study 39 was $\pm 4 \mu \mathrm{S} / \mathrm{cm}$ of the median value of 13.0. Using this criterion, 97.3 percent of responding site operators met the goals for specific-conductance measurements. 
Table 1. Site-operator responses and summary statistics for the 1997 intersite-comparison program

\begin{tabular}{lcc}
\hline \multicolumn{1}{c}{ Site-operator responses } & Intersite-comparison study number \\
\cline { 2 - 3 } & 39 & $\mathbf{4 0}$ \\
\hline Number of site operators receiving samples & 195 & 195 \\
Number of site operators submitting pH values by closing date of study & 186 & 183 \\
Number of site operators submitting specific-conductance values by closing date of study & 184 & 184 \\
Site operators responding late & 1 & 0 \\
Number of nonresponding site operators & 2 & 4 \\
Sites that were not in operation & 3 & 1 \\
Site operators reporting equipment problems: & & 8 \\
pH meter/electrode completely inoperable & 8 & 0 \\
pH meter/electrode problems & 0 & 7 \\
Specific-conductance probe/meter completely inoperable & 8 & 0 \\
Specific-conductance probe/meter problems & 0 & $4.12,4.09$ \\
Median pH, target pH & $4.78,4.75$ & 157 \\
Number of responding sites that met the pH accuracy goals & 154 & $4.04,4.18$ \\
Accuracy goals for pH: lower and upper acceptable values & $4.62,4.93$ & 85.8 \\
Percentage of responding sites that met the pH accuracy goals & 83.7 & 0.030 \\
F-pseudosigma for pH & 0.052 & $33.9,34.2$ \\
Median specific conductance, target specific conductance, in microsiemens per centimeter at & $13.0,13.0$ & 170 \\
$\quad$ 25 degrees Celsius & & 179 \\
Number of responding sites that met the specific-conductance accuracy goals & $9.0,17.0$ & 97.3 \\
Accuracy goals for specific conductance: lower and upper acceptable values & 0.519 & $29.9,37.9$ \\
Percentage of responding sites that met the specific-conductance accuracy goals & & 92.4 \\
F-pseudosigma for specific conductance & & 1.446 \\
\hline
\end{tabular}

The reference solution used in intersitecomparison study 40 had a target $\mathrm{pH}$ of 4.09 and a target specific conductance of $34.2 \mu \mathrm{S} / \mathrm{cm}$. One hundred and fifty-seven site operators, 85.8 percent, met the $\mathrm{pH}$ accuracy goals of 4.04 to 4.18 . The median specific conductance was $33.9 \mu \mathrm{S} / \mathrm{cm}$, with 92.4 percent of site operators achieving the accuracy goal of $\pm 4 \mu \mathrm{S} / \mathrm{cm}$ of the median value.

For intersite-comparison study 41 , the reference solution target $\mathrm{pH}$ was 4.64 and the target specific conductance was $19.0 \mu \mathrm{S} / \mathrm{cm}$ after the solution was spiked with potassium chloride. A total of 170 site operators ( 90.9 percent) met the $\mathrm{pH}$ accuracy goals of 4.48 to $4.74 \mathrm{pH}$ units. The median specificconductance value was $20.2 ; 94.7$ percent of site operators achieved the median $\pm 4 \mu \mathrm{S} / \mathrm{cm}$ accuracy goal.

The reference solution used in intersitecomparison study 42 had a target $\mathrm{pH}$ of 4.39 and a target specific conductance of $25.0 \mu \mathrm{S} / \mathrm{cm}$ (the solution was spiked with potassium chloride). One hundred and fifty-nine site operators ( 90.3 percent) met the $\mathrm{pH}$ accuracy goal of 4.27 to 4.47 . The median specific conductance was 26.9 , with 96.0 percent of site operators achieving the median $\pm 4 \mu \mathrm{S} / \mathrm{cm}$ accuracy goal.

For intersite-comparison study 43 , the reference solution target $\mathrm{pH}$ was 4.83 and the target specific conductance was $I 7.0 \mu \mathrm{S} / \mathrm{cm}$ after the solution was spiked with potassium chloride. One hundred and sixty-two site operators ( 89.5 percent) met the $\mathrm{pH}$ accuracy goals of 4.63 to $4.95 \mathrm{pH}$ units. The median specific-conductance value was 17.6; 99.4 percent of site operators achieved the median $\pm 4 \mu \mathrm{S} / \mathrm{cm}$ accuracy goal.

Figure 2 shows the results of $\mathrm{pH}$ and specificconductance values for all participating site operators in intersite-comparison studies 39 and 40; the results for studies 41 and 42 are shown in figure 3 , and results for study 43 are shown in figure 4 . The shaded areas on the scatterplots delineate the $\mathrm{pH}$ and specific-conductance values for those site operators meeting the accuracy goals for $\mathrm{pH}$, specific conductance, or both measurements; values outside the shaded areas are those not meeting the accuracy goals. The percentile distributions for the reported $\mathrm{pH}$ and specific-conductance values for studies 39 through 43 are listed in figure 5. 
Table 2. Site-operator responses and summary statistics for the 1998-99 intersite-comparison program

\begin{tabular}{lccc}
\hline \multicolumn{1}{c}{ Site-operator responses } & \multicolumn{2}{c}{ Intersite-comparison study number } \\
\cline { 2 - 4 } & 41 & 42 & 43 \\
\hline Number of site operators receiving samples & 199 & 198 & 209 \\
Number of site operators submitting pH values by closing date of study & 187 & 176 & 181 \\
Number of site operators submitting specific conductance values by closing date of study & 187 & 176 & 180 \\
Site operators responding late & 0 & 7 & 16 \\
Number of non-responding site operators & 6 & 7 & 11 \\
Sites that were not in operation & 2 & 1 & 0 \\
Site operators reporting equipment problems: & 6 & 2 & 2 \\
pH meter/electrode completely inoperable & 0 & 0 & 0 \\
pH meter/electrode problems & 6 & 8 & 4 \\
Specific conductance probe/meter completely inoperable & 0 & 0 & 0 \\
Specific conductance probe/meter problems & $4.62,4.64$ & $4.38,4.39$ & $4.80,4.83$ \\
Median pH, target pH & 170 & 159 & 162 \\
Number of responding sites that met the pH accuracy goals & $4.48,4.74$ & $4.27,4.47$ & $4.63,4.95$ \\
Accuracy goals for pH: lower and upper acceptable values & 90.9 & 90.3 & 89.5 \\
Percentage of responding sites that met the pH accuracy goals & 0.044 & 0.044 & 0.067 \\
F-pseudosigma for pH & $20.2,19.0$ & $26.9,25.0$ & $17.6,17.0$ \\
Median specific conductance, target specific conductance, in microsiemens per & & & 178 \\
centimeter at 25 degrees Celsius & 177 & 169 & $17.6,21.6$ \\
Number of responding sites that met the specific conductance accuracy goals & $16.2,24.2$ & $22.9,30.9$ & 13.9 \\
Accuracy goals for specific conductance: lower and upper acceptable values & 94.7 & 96.0 & 99.4 \\
Percentage of responding sites that met the specific conductance accuracy goals & 0.667 & 0.964 & 0.445 \\
F-pseudosigma for specific conductance & &
\end{tabular}

\section{Intersite-Comparison Study Followup Program}

After the initial intersite-comparison study results are tabulated, the results from site operators who do not meet the $\mathrm{pH}$ accuracy goals are evaluated further. The purpose of the followup program is to help site operators identify and resolve sources of measurement difficulty and produce improved weekly data for the NADP/NTN. Each operator that fails to meet the accuracy goals is placed into one of four followup categories (discussed later) based on their performance in the current study and in the two preceding intersite studies. For the followup evaluation, the site operator's reported values are converted into standardized $\mathrm{z}$-values. Z-values are analogous to Z-scores described by Iman and Conover (1983). To compute a $\mathrm{z}$-value, nonparametric estimators replace the traditional parametric estimators used by Iman and Conover. The formulas for $\mathrm{z}$-scores and $\mathrm{z}$-values are:

$$
\mathrm{z}-\mathrm{score}=\frac{x-\bar{x}}{\mathrm{~S}}
$$

and

$$
\mathrm{z}-\text { value }=\frac{x-\tilde{x}}{\mathrm{fps}}
$$

where

$$
\begin{aligned}
\mathrm{x}= & \text { an individual observation; } \\
\bar{x}= & \text { the mean of all observations; } \\
\tilde{x}= & \text { the median of all observations; } \\
\mathrm{S}= & \text { standard deviation of all observations; and } \\
\mathrm{fps}= & \mathrm{f} \text {-pseudosigma of all observations computed } \\
& \text { by: } \\
& \frac{\text { upper quartile }- \text { lower quartile }}{1.349}
\end{aligned}
$$

By using standardized z-values, each site operator's performance relative to all other site operators is evaluated objectively. The standardized $\mathrm{z}$-values take into account the amount by which $\mathrm{pH}$-measurement accuracy goals are missed, given the relative difficulty of measuring the $\mathrm{pH}$ of the solution. The relative difficulty of measuring the $\mathrm{pH}$ is inversely related to the hydrogen-ion concentration of the solution: the lower the hydrogen-ion concentration, the more difficult the measurement. A cumulative $\mathrm{z}$-value total for the three 
INTERSITE COMPARISON STUDY NUMBER 39 -June 1997

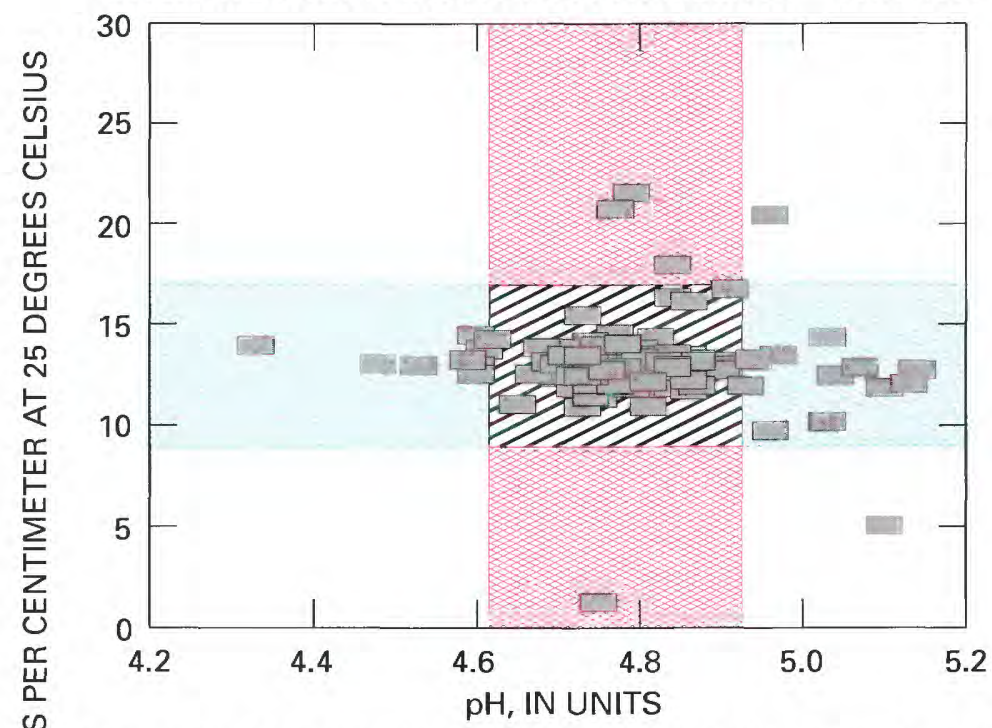

INTERSITE COMPARISON STUDY NUMBER 40 -December 1997

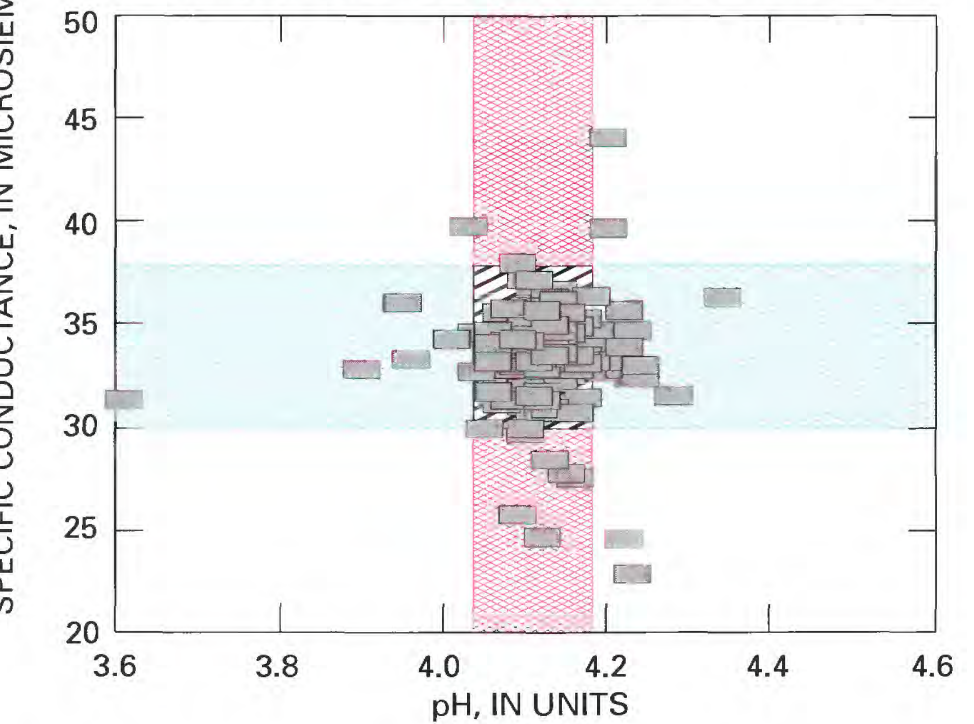

\section{EXPLANATION}

Met National Atmospheric Deposition Program/National Trends Network (NADP/NTN) accuracy goals for $\mathrm{pH}$ only

Met NADP/NTN accuracy goals for $\mathrm{pH}$ and specific conductance

Met NADP/NTN accuracy goals for specific conductance only

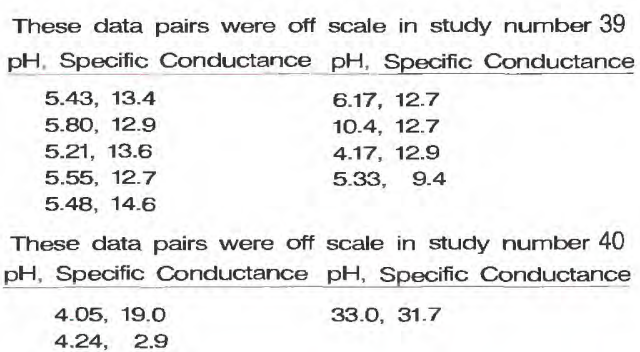

Figure 2. Distribution of $\mathrm{pH}$ and specific-conductance values for intersite-comparison studies 39 and 40, completed in 1997. 
INTERSITE COMPARISON STUDY NUMBER 41 -June 1998

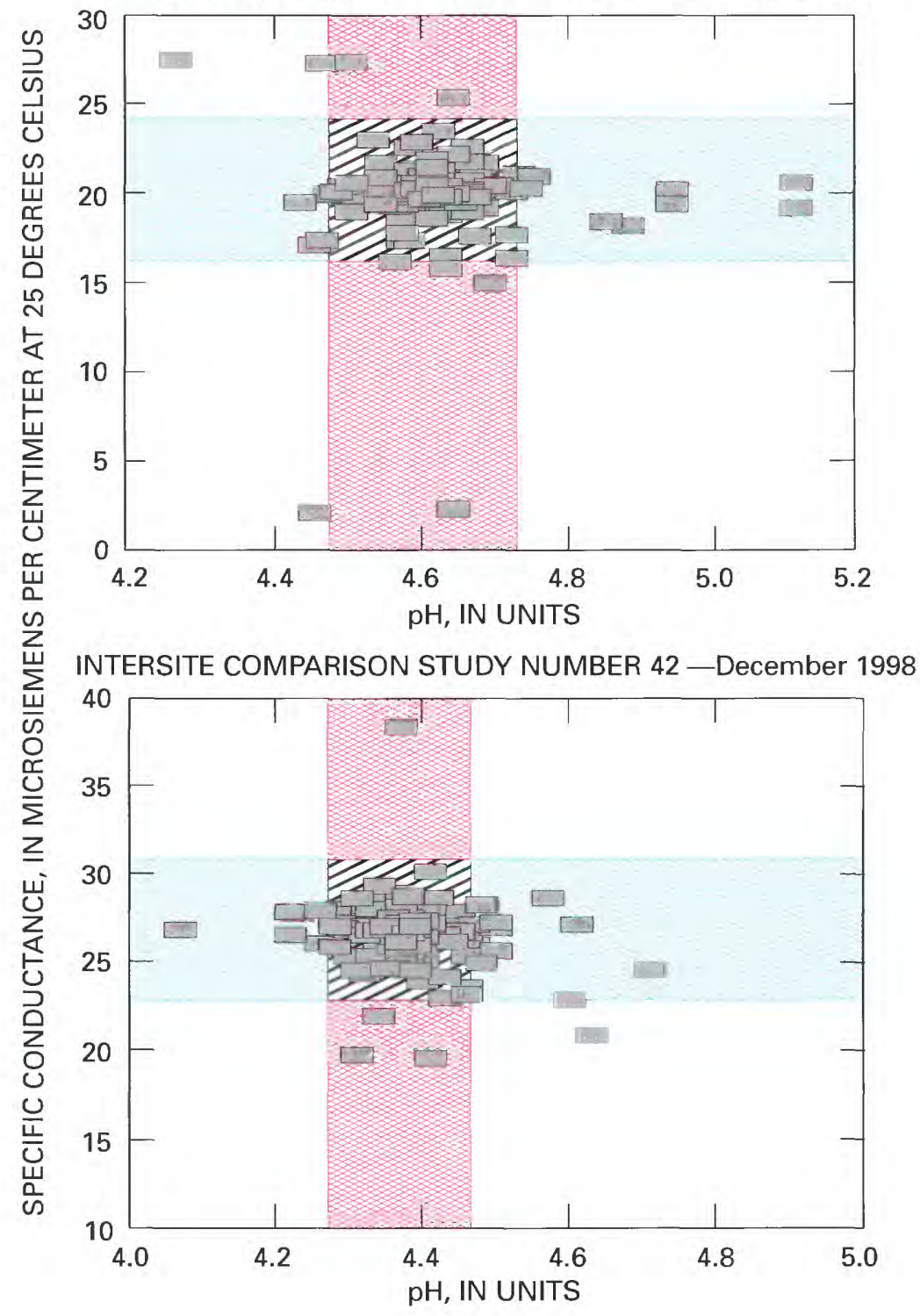

\section{EXPLANATION}

Met National Atmospheric Deposition Program/National Trends Network (NADP/NTN) accuracy goals for $\mathrm{pH}$ only

Met NADP/NTN accuracy goals for $\mathrm{pH}$ and specific conductance

Met NADP/NTN accuracy goals for specific conductance only
These data pairs were off scale in study number 41 $\mathrm{pH}$, Specific Conductance $\mathrm{pH}$, Specific Conductance $6.00,16.9$ $3.87,17.5$ $4.40,85.1 \quad 4.69,34.0$

These data pairs were off scale in study number 42 $\mathrm{pH}$, Specific Conductance $\mathrm{pH}$, Specific Conductance 3.69, 27.2 $4.44, \quad 2.6$

Figure 3. Distribution of $\mathrm{pH}$ and specific-conductance values for intersite-comparison studies 41 and 42, completed in 1998. 


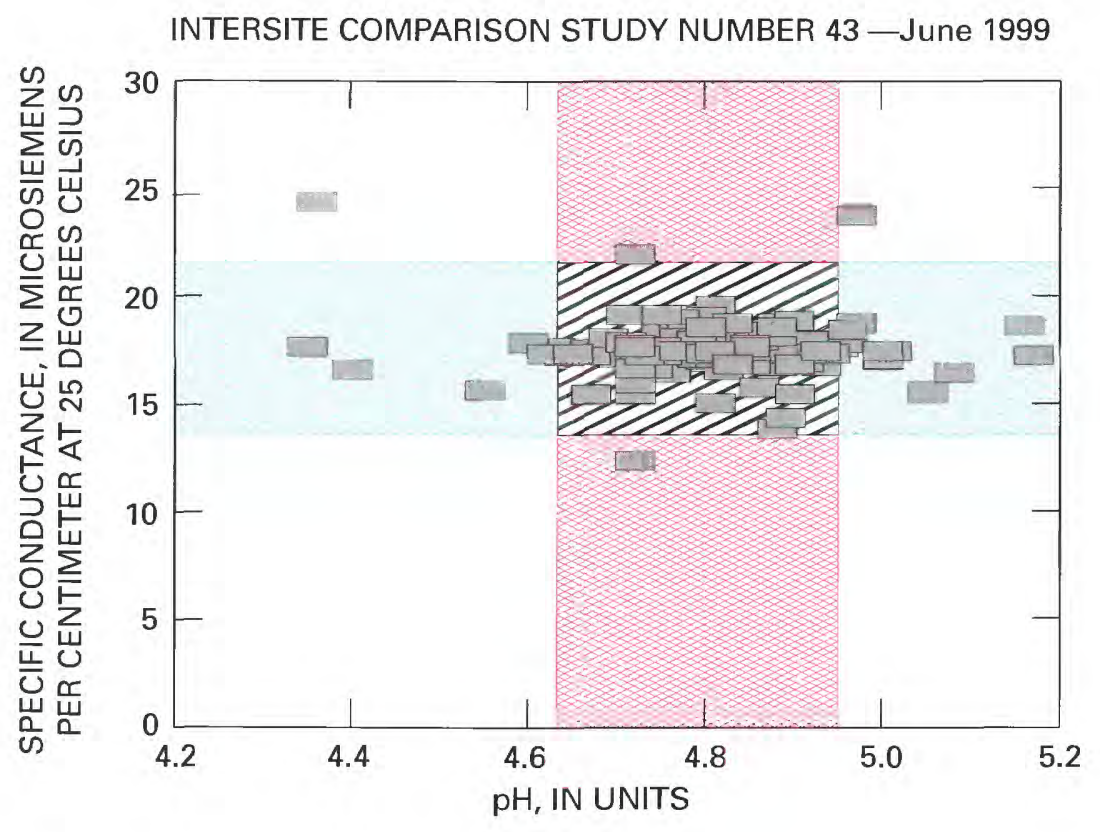

These data pairs were off scale in study number 43
$\begin{array}{ll}\mathrm{pH} \text {. Specific Conductance } & \mathrm{pH}, \text { Specific Conductance } \\ 5.50,18.0 & 5.39,17.0 \\ 5.58,18.1 & 6.17,-6.0 \\ 5.63,20.6 & \end{array}$

EXPLANATION

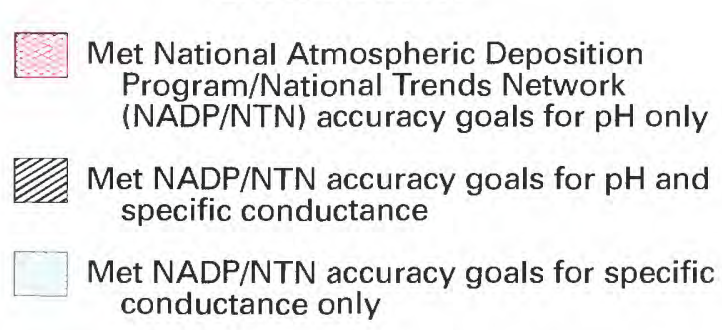

Figure 4. Distribution of $\mathrm{pH}$ and specific-conductance values for intersitecomparison study 43, completed in 1999.

most recent studies is used to place each site operator failing to meet the accuracy goals into one of these four followup study categories:

Level 1. Operators receive a letter stating the problem with the recent results and suggestions for troubleshooting and improving field techniques.

Level 2. Operators are asked to remeasure and resubmit results for the current intersite study.

Level 3. Operators are given one additional check sample to measure.

Level 4. Operators are given two additional check samples to measure.
Higher level categories require additional measurements: Level 3 site operators remeasure the original sample and measure an additional check sample. Level 4 site operators remeasure the original sample and measure two additional check samples. The additional check samples sent to Level 3 and 4 site operators are past intersite-comparison study samples that have been stored at $4^{\circ} \mathrm{C}$ in their original unopened bottles. Previous studies (Gordon and others, 1995; Peden and Skowron, 1978) indicate that the stability of hydrogen-ion concentration over time is sufficient to allow the use of previous intersite samples in the followup studies. 


\begin{tabular}{|c|c|c|c|c|c|c|c|c|}
\hline \multirow{7}{*}{ 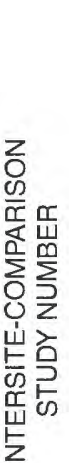 } & & \multicolumn{6}{|c|}{ PERCENTILES } & \\
\hline & & 5 & 10 & 25 & 50 & 75 & 90 & 95 \\
\hline & 39 & 4.62 & 4.70 & 4.74 & 4.78 & 4.83 & 4.96 & 5.13 \\
\hline & 40 & 4.05 & 4.07 & 4.10 & 4.12 & 4.15 & 4.19 & 4.23 \\
\hline & 41 & 4.48 & 4.52 & 4.58 & 4.62 & 4.65 & 4.69 & 4.74 \\
\hline & 42 & 4.28 & 4.30 & 4.34 & 4.38 & 4.40 & 4.45 & 4.48 \\
\hline & 43 & 4.64 & 4.70 & 4.76 & 4.80 & 4.85 & 4.92 & 5.00 \\
\hline
\end{tabular}

\begin{tabular}{|c|c|c|c|c|c|c|c|c|}
\hline & & FOR & $\mathrm{DEClFI}$ & $\begin{array}{l}\text { PER } \\
-\mathrm{CON}\end{array}$ & $\begin{array}{l}\text { CEN] } \\
\text { UCTAN }\end{array}$ & $\begin{array}{l}\text { LES } \\
\text { LE ME }\end{array}$ & SUREI & ENTS \\
\hline & & 5 & 10 & 25 & 50 & 75 & 90 & 95 \\
\hline $\begin{array}{l}Z \\
0 \\
\frac{\infty}{n}\end{array}$ & 39 & 11.5 & 12.0 & 12.7 & 13.0 & 13.4 & 13.9 & 14.6 \\
\hline 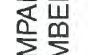 & 40 & 29.7 & 31.1 & 32.8 & 33.9 & 34.7 & 35.7 & 36.3 \\
\hline Оิईz & 41 & 17.4 & 18.6 & 19.7 & 20.2 & 20.6 & 21.4 & 22.6 \\
\hline 崖它 & 42 & 24.0 & 24.7 & 26.1 & 26.9 & 27.3 & 28.0 & 28.5 \\
\hline 采怘 & 43 & 15.8 & 16.7 & 17.3 & 17.6 & 17.9 & 18.6 & 19.0 \\
\hline
\end{tabular}

\section{SPECIFIC CONDUCTANCE, IN MICROSIEMENS PER CENTIMETER AT 25 DEGREES CELSIUS}

Figure 5. Percentiles for $\mathrm{pH}$ and specific conductance from intersite-comparison studies 39 through 43.

In intersite-comparison study 39, 37 site operators were included in the followup study. Twenty-four of these site operators were asked to perform additional $\mathrm{pH}$ measurements. Ten of these site operators met the accuracy goals for all $\mathrm{pH}$ measurements, and five site operators did not respond. Intersite-comparison study 41 included 18 site operators in the followup study, and 16 site operators were included in Levels 2, 3, and 4. Five of the 12 site operators that remeasured the $\mathrm{pH}$ of the remaining intersite solution met the accuracy goals. The intersite-comparison study 42 followup study included 33 site operators (18 percent of all site operators in the original study), and 32 of these site operators were asked to perform additional $\mathrm{pH}$ measurements. Five of these 32 site operators met all accuracy goals for the $\mathrm{pH}$ measurements, and 10 site operators did not respond. Figure 6 summarizes the followup results for studies 39,41 , and 42 . Followup studies were not completed for intersite-comparison studies 40 and 43.

\section{BLIND-AUDIT PROGRAM}

The blind-audit program evaluates the effects of routine handling, processing, and shipping of wet-deposition samples on analyte bias and precision. Biased analytical data can result from contamination introduced during sample shipping, handling, or processing. In the blind-audit program, site operators submit a portion of a synthetic precipitation sample disguised as a natural precipitation sample to the Central Analytical Laboratory (CAL) for analysis. The normal processing and handling steps of a regular weekly sample are applied to the portion of the blindaudit sample disguised as a natural precipitation sample. The remaining minimally handled portion of the blind-audit sample is sent to the CAL for analysis in a separate mailer and is analyzed independently of the portion subject to actual weekly precipitationsample processing and handling. Contact with the sample-collection container and routine handling, and processing procedures applied to wet-deposition samples have been identified as sources of contamination (Gordon, 1999; Nilles and others, 1995).

In each of the first two quarters of 1997, 32 blind-audit samples were sent to the operators of selected NADP/NTN sites. The number of blind-audit samples mailed to site operators was reduced to 25 per quarter beginning in the third quarter of 1997 . The reduction in the number of blind-audit samples coincided with the initiation of full-scale operation of the field-audit program. Sites selected on a quarterly basis are chosen to ensure a uniform geographic distribution throughout the United States. After a site has been selected for the blind-audit program (assuming successful participation), the site is not selected again for the blind-audit program until the operators of all other NADP/NTN sites have participated. All NADP/NTN sites were sent at least one blind-audit sample between 1997 and 1999.

The solutions used in the blind-audit program are intended to replicate the range of analyte concentrations typically found in natural NADP/NTN precipitation 

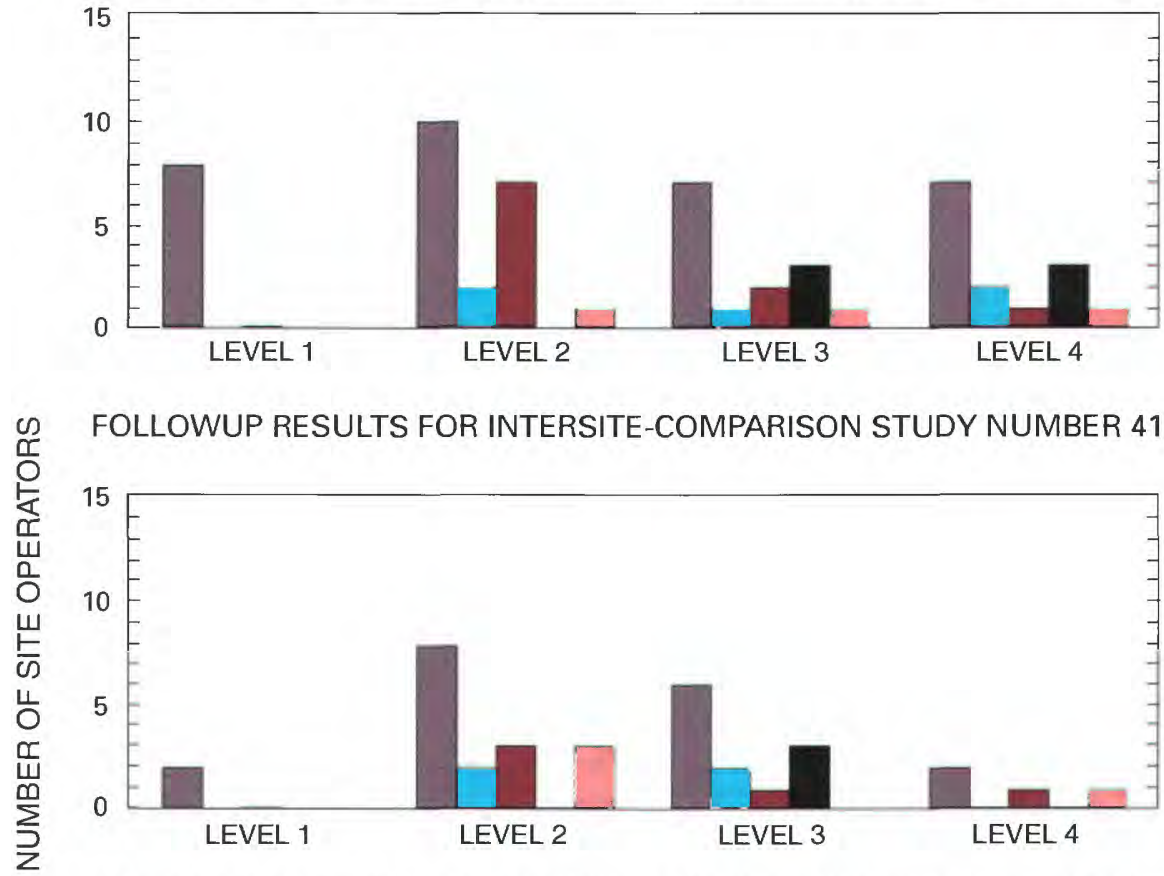

FOLLOWUP RESULTS FOR INTERSITE-COMPARISON STUDY NUMBER 42

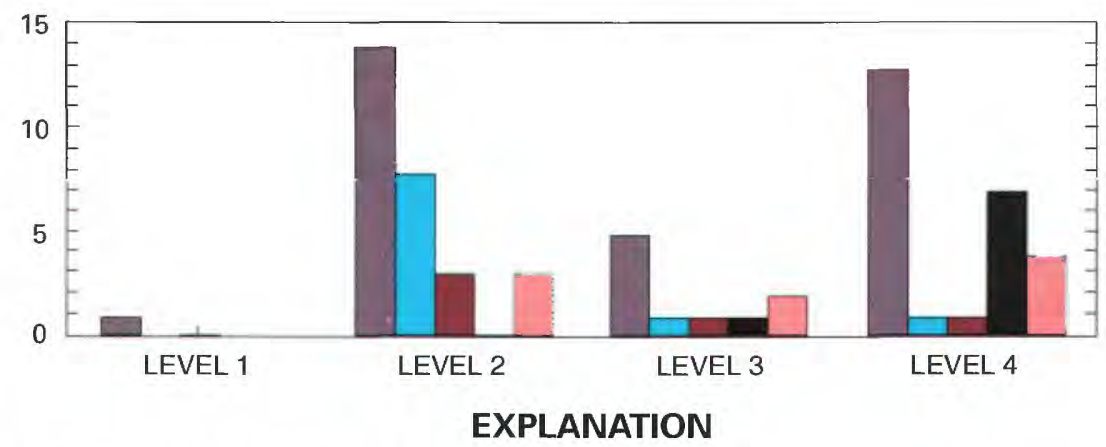

Number of site operators that:

Were asked to participate in the followup study

Did not participate in the followup study

Successfully met the accuracy goals for the followup study

\section{LEVEL 1 followup:}

(1) Letter discussing common sources of measurement errors

\section{LEVEL 2 followup:}

(1) Letter discussing common sources of measurement errors

(2) Request that site operator reanalyze the remaining portion of the test solution
Had mixed success meeting the accuracy goals for the followup study

Did not meet the accuracy goals for the followup study

\section{LEVEL 3 followup:}

(1) Letter discussing common sources of measurement errors

(2) Request that site operator reanalyze the remaining portion of the test solution

(3) One additional aliquot of test solution

\section{LEVEL 4 followup:}

(1) Letter discussing common sources of measurement errors

(2) Request that site operator reanalyze the remaining portion of the test solution

(3) Two additional aliquots of test solution

Figure 6. Followup study results for intersite-comparison studies 39,41 , and 42 . 
samples. The median analyte-concentration values for the solutions used in the blind-audit program (other than Ultrapure deionized water) were between the 25 th and 75th percentiles of natural precipitation samples collected at NADP/NTN sites. Many of the solutions used in the blind-audit program also are used in the field-audit and interlaboratory-comparison programs. Solutions used in the blind-audit, field-audit, and interlaboratory-comparison programs, the names of the agencies that prepared them, and any special remarks about each solution are listed in table 3 . The target values for these solutions are presented in table 4 .

Three different sample volumes were distributed for one of the sample matrices. Throughout 1997 and in the first quarter of 1998, sample sizes of 250, 500, or $1,000 \mathrm{~mL}$ of the USGS solution were sent to operators of selected sites to assess volume-related effects on biases. Beginning in the second quarter of 1998, the supply of USGS solution was depleted and replaced with the SP-3 solution for the remainder of 1998. Starting with the first quarter in 1999, SP-5 solution was used in place of the SP-3 solution to assess the volume-related effects. The volume of USGS, SP-3, or $\mathrm{SP}-5$ solution that a site received was determined on a random basis. Additional information regarding the blind-audit program is available in previous reports (Gordon, 1999; Gordon and others, 1997; See and others, 1990). Figure 7 outlines the components of the blind-audit program, from sample preparation to distribution of interpretive reports.

Site operators are provided detailed blindaudit sample-processing instructions. The handling and processing steps of a regular weekly precipitation sample are duplicated as closely as possible. The instructions prescribe that 75 percent of the blind-audit sample be poured into a standard, clean, NADP/NTN 13-L polyethylene collection bucket and processed as if it were the natural precipitation sample from the previous week. The blind-audit samples sent to the site operators are marked with a line specifying the quantity to pour into the clean bucket. This poured-out portion of the blind-audit sample is referred to as the bucket sample. The operator determines the weight of the bucket containing 75 percent of the blind-audit sample. After a minimum residence time of 24 hours, the sample is transferred from the bucket into a clean 1-L, high-density polyethylene (HDPE) shipping bottle. The operator pours a small amount (approximately $20 \mathrm{~mL}$ ) from the 1-L bottle into clean vials and measures the $\mathrm{pH}$ and specific conductance following standard procedures. Using a fictitious NADP/NTN field-observer report form, the shipping bottle is disguised as a routine natural precipitation sample and submitted to the CAL for analysis. The site operator

Table 3. Solutions used in the 1997-99 blind-audit, field-audit, and interlaboratory-comparison programs

$[\mathrm{M} \Omega$ megohm]

\begin{tabular}{|c|c|c|}
\hline Solution & Preparing agency & Remarks \\
\hline CAL 4.3 & $\begin{array}{l}\text { Illinois State Water Survey, } \\
\text { Central Analytical Laboratory }\end{array}$ & Dilute acid solution. \\
\hline Ultrapure & U.S. Geological Survey & Deionized water with a measured resistivity greater than $16.7 \mathrm{M} \Omega$ \\
\hline $\begin{array}{l}\text { USGS } \\
\text { SP-1 } \\
\text { SP-2 } \\
\text { SP-3 } \\
\text { SP-4 } \\
\text { SP-5 }\end{array}$ & U.S. Geological Survey & $\begin{array}{l}\text { Prepared from dissolved salts and deionized water. SP-1, SP-3, and SP-4 solutions } \\
\text { prepared exclusively by the USGS. USGS prepared the SP-2 and SP-5 solutions } \\
\text { through } 1998 \text {. }\end{array}$ \\
\hline P-96 & U.S. Geological Survey & $\begin{array}{l}\text { Prepared from a low-ionic strength reference sample collected by the Standard } \\
\text { Reference Sample Project. Diluted with deionized water to achieve concentration } \\
\text { levels similar to those found in precipitation. }\end{array}$ \\
\hline 2694-A-II & $\begin{array}{l}\text { National Institute of Standards } \\
\text { and Technology }\end{array}$ & $\begin{array}{l}\text { Supplied as certified reference solution; supplier has discontinued production and } \\
\text { the solution was last used in } 1997 .\end{array}$ \\
\hline
\end{tabular}




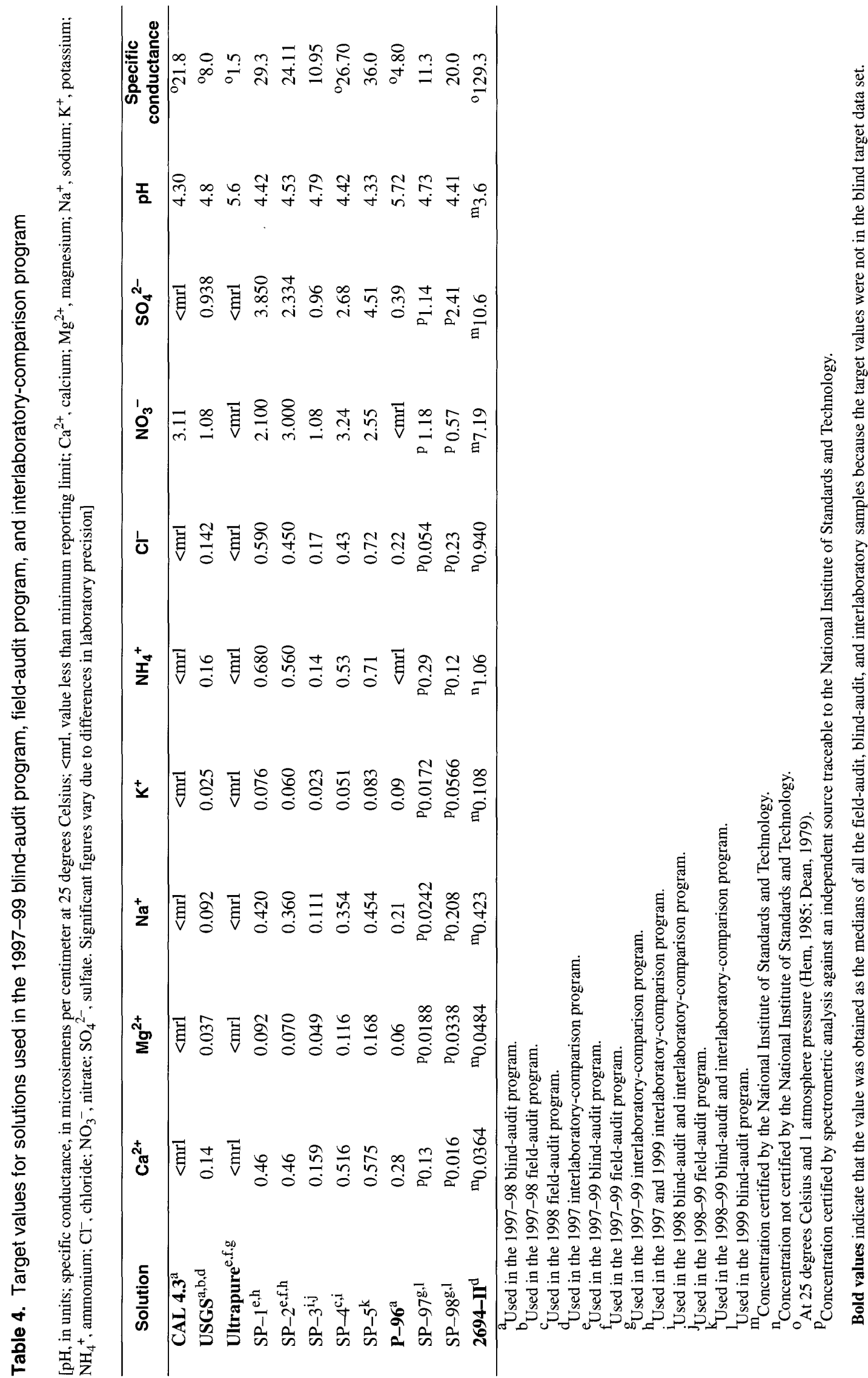




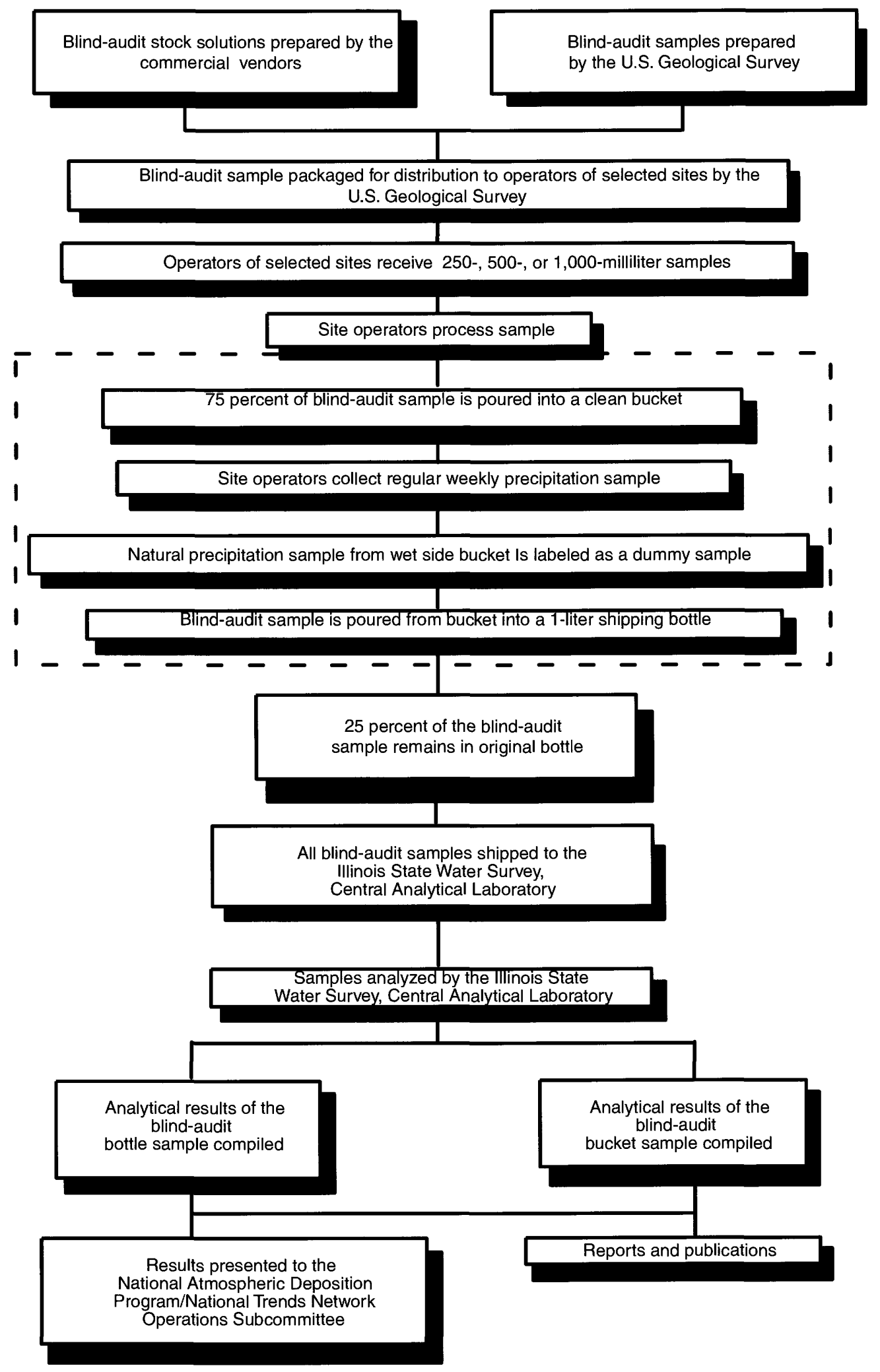

Figure 7. Blind-audit program of the U.S. Geological Survey. 
also collects a natural precipitation sample during the assigned week for submitting the blind-audit sample. The natural precipitation sample is submitted to the CAL using a "dummy" field-observer report form. The remaining 25 percent of the blind-audit sample, still in the original sample bottle, is returned to the CAL for analysis. This portion of the blind-audit sample is referred to as the bottle sample. In order to keep their identities concealed, the natural precipitation sample and the two portions of the blind-audit sample are all shipped separately to the CAL. A report by Gordon and others (1997) contains additional details on the submission of blind-audit samples.

Because of the order in which samples and fieldobserver report forms are processed, it is unlikely that the CAL staff identifies individual samples as external QA samples. Information concerning sample chemical composition is not provided to the CAL staff doing the analyses or to the site operators doing the processing. When the bottle portion of a blind-audit sample is submitted to the CAL, only the sample-processing group (the $\mathrm{pH}$, specific conductance, and filtering analysts) of the laboratory staff recognizes that it is not a natural NADP/NTN precipitation sample.

The blind-audit program is designed so that the CAL staff receiving and analyzing the natural precipitation sample is not able to identify the site from which the sample has been sent. After all the analyses for the bucket and bottle portion of the blind-audit sample and for the natural precipitation sample are completed, the identity of each of these samples is disclosed to the CAL Data Manager. The NADP/NTN database is then corrected by matching the proper analytical data with each sample.

\section{Data Analysis}

Differences in analyte concentrations between the bucket and bottle portions can result from sample handling, shipping, and processing protocols and can lead to analytical bias and variability. In 1997 , complete bucket and bottle analyses were available for 101 of the 114 blind-audit samples sent to participating site operators. In 1998, complete bucket and bottle analyses were available for 88 of the 100 blindaudit samples sent to participating operators. In 1999, complete bucket and bottle analyses were available for 95 of the 102 blind-audit samples sent to participating operators.
When physical evidence of contamination is discovered and the chemistry is unusual, CAL assigns the natural precipitation samples a " $C$ " code to indicate the sample is contaminated (James, 1996). Regardless of sample chemistry, all quality-assurance samples (including the bucket and bottle portions of blind-audit samples) containing extrinsic material are assigned a "C" code. Using the Wilcoxon signed-rank test to compare the two groups of blind-audit samples (C-coded samples and samples without "C" codes) for all major ions, only one statistically significant difference was found (potassium) at the $\alpha=0.05$ level when the C-coded samples were included that was not found when the C-coded samples were not included during 1997 through 1999 (see table 6). These results are comparable to those from past years, comparing Ccoded (contaminated) and uncontaminated blind-audit analyses. Data from C-coded samples during 1997 through 1999 were therefore included in the overall statistical analysis of blind-audit samples for each of these 3 years.

\section{Analytical Bias and Precision}

Paired bucket minus bottle differences were calculated to determine analytical bias. Before determining paired bucket minus bottIe differences, bucket and bottle values reported as less than the minimum reporting limit (MRL) were set equal to one-half the MRL. Alternatively, analyte concentrations reported as less than the MRL were set equal to the MRL and then to zero in order to determine if the results of the Wilcoxon signed-rank test for bias would be different. Regardless of whether the values less than the MRL were set equal to zero, one-half the MRL, or equal to the MRL, the results of the Wilcoxon signed-rank test for bias essentially were the same with only a couple of minor exceptions. The handling of values less than the MRL for this report is consistent with the handling of values less the MRL in previous reports (Gordon, 1999; Gordon and others, 1997). The median paired bucket minus bottle differences for all analytes during 1997 through 1999 are presented in table 5, along with the minimum and maximum values, the upper and lower quartiles, and the interquartile range. The median paired differences were consistently close to zero for the major ions during 1997 through 1999, indicating a lack of bias.

Boxplots in figure 8 graphically depict the paired bucket minus bottle concentration differences for all the major ions as well as $\mathrm{pH}$ and specific conductance. The 
Table 5. Selected statistics for the paired bucket-sample concentration minus bottle-sample concentration differences in the blind-audit program during 1997 through 1999

[All units in milligrams per liter except hydrogen ion, in microequivalents per liter, and specific conductance, in microsiemens per centimeter at 25 degrees Celsius; N, number of samples: Q1. the lower quartile in the data distribution; Q3, the upper quartile in the data distribution; interquartile range, the difference between the upper and lower quartiles in the distribution (Q3 minus Q1)]

\begin{tabular}{|c|c|c|c|c|c|c|c|}
\hline \multirow{2}{*}{ Analyte } & \multirow{2}{*}{$\mathbf{N}$} & \multirow{2}{*}{ Minimum } & \multirow{2}{*}{ Median } & \multicolumn{2}{|c|}{ Quartiles } & \multirow{2}{*}{ Maximum } & \multirow{2}{*}{$\begin{array}{l}\text { Interquartile } \\
\text { range }\end{array}$} \\
\hline & & & & Q1 & Q3 & & \\
\hline Calcium & 284 & -0.567 & 0.005 & -0.001 & 0.016 & 0.462 & 0.017 \\
\hline Magnesium & 285 & -0.172 & 0.001 & 0.000 & 0.003 & 0.106 & 0.003 \\
\hline Sodium & 285 & -0.437 & 0.001 & -0.008 & 0.007 & 0.345 & 0.015 \\
\hline Potassium & 285 & -0.121 & 0.002 & 0.000 & 0.005 & 1.478 & 0.005 \\
\hline Ammonium & 285 & -0.620 & 0.000 & -0.010 & 0.010 & 0.470 & 0.020 \\
\hline Nitrate & 285 & -2.505 & 0.000 & -0.010 & 0.030 & 2.530 & 0.040 \\
\hline Sulfate & 285 & -4.485 & 0.010 & 0.000 & 0.040 & 2.325 & 0.040 \\
\hline Hydrogen ion & 286 & -40.090 & -1.086 & -2.924 & 0.027 & 26.628 & 2.951 \\
\hline Specific conductance & 286 & -33.100 & -0.200 & -0.900 & 0.200 & 12.100 & 1.100 \\
\hline
\end{tabular}

upper and lower lines defining the "box" portions of the graphs depict the interquartile range of the differences for each analyte. The "whisker" portions of the boxplots are defined by the largest value within \pm 1.5 times the interquartile range extending from either the top or bottom of the "box" portion. Outliers are clustered closer to the boxplots for almost all of the major ions than in previous years (Gordon. 1999), reflecting the sharp decrease in variability between matched blindaudit sample pair results. In terms of chemical significance, the change in the NADP/NTN shipping protocol in 1994 continues to have a major effect. Since the 1994 protocol change, there is no longer a significant loss of hydrogen ion, an observation first noted in the report on 1994 external quality-assurance results (Gordon and others, 1997). The quartiles of the hydrogen ion differences indicated that 50 percent of the samples experienced a change in hydrogen-ion concentration of between -2.924 and $+0.027 \mu \mathrm{eq} / \mathrm{L}$ (table 5), which is only a small percentage of the hydrogen-ion concentration present in the reference samples used in the program. Subtle improvements in shipping, handling, and processing procedures in 1997-99 (such as improved sample splitting and filtering techniques and enhanced quality control of bag supplies) may have further reduced the variability remaining in blind-audit results (and by inference, in the weekly NADP/NTN sample chemistry results) subsequent to the striking improvements caused by the 1994 protocol change.

A Wilcoxon signed-rank test (Conover, 1980) and paired t-test (Kanji, 1993) were used to determine if statistically significant differences existed between the analyte concentrations measured for the paired bucket and bottle portions of the blind-audit samples submitted during 1997 through 1999. Analytical results were evaluated for evidence of systematic bias by using both the paired t-test and the Wilcoxon signed-rank test because both offer different advantages. The paired $\mathbf{t}$-test is a more powerful test than the Wilcoxon signed-rank test for paired observations (Kanji, 1993). However, the paired t-test loses some of its power if the populations are not normal (Kanji, 1993). Blind-audit samples that had paired analyte determinations were included in both statistical analyses. At a significance level of $\alpha=0.05$, the Wilcoxon signed-rank test indicated there was only statistically significant bias for potassium during 1997 through 1999 (test 2, table 6), and with a median paired blind-audit difference of +0.002 (table 5) for potassium, even this one lone statistically significant bias was not large enough to be chemically significant (that is, large enough to be important to a chemist or hydrologist evaluating the data). The t-test indicated a positive bias for calcium and chloride and a negative bias for hydrogen ion during 1997 through 1999 (table 6). The negative bias for ammonium and sodium found in the 1995-96 results (Gordon, 1999), reflecting the tendency for the portion of the blind-audit samples exposed to all of the handling and processing steps to have a lower concentration of these analytes than the minimally handled control portion of the blind-audit samples, appears to have been eliminated during 1997 through 1999.

During 1997 through 1999, the median bucket minus bottle differences for major ions in the pooled results from all blind-audit samples ranged from 


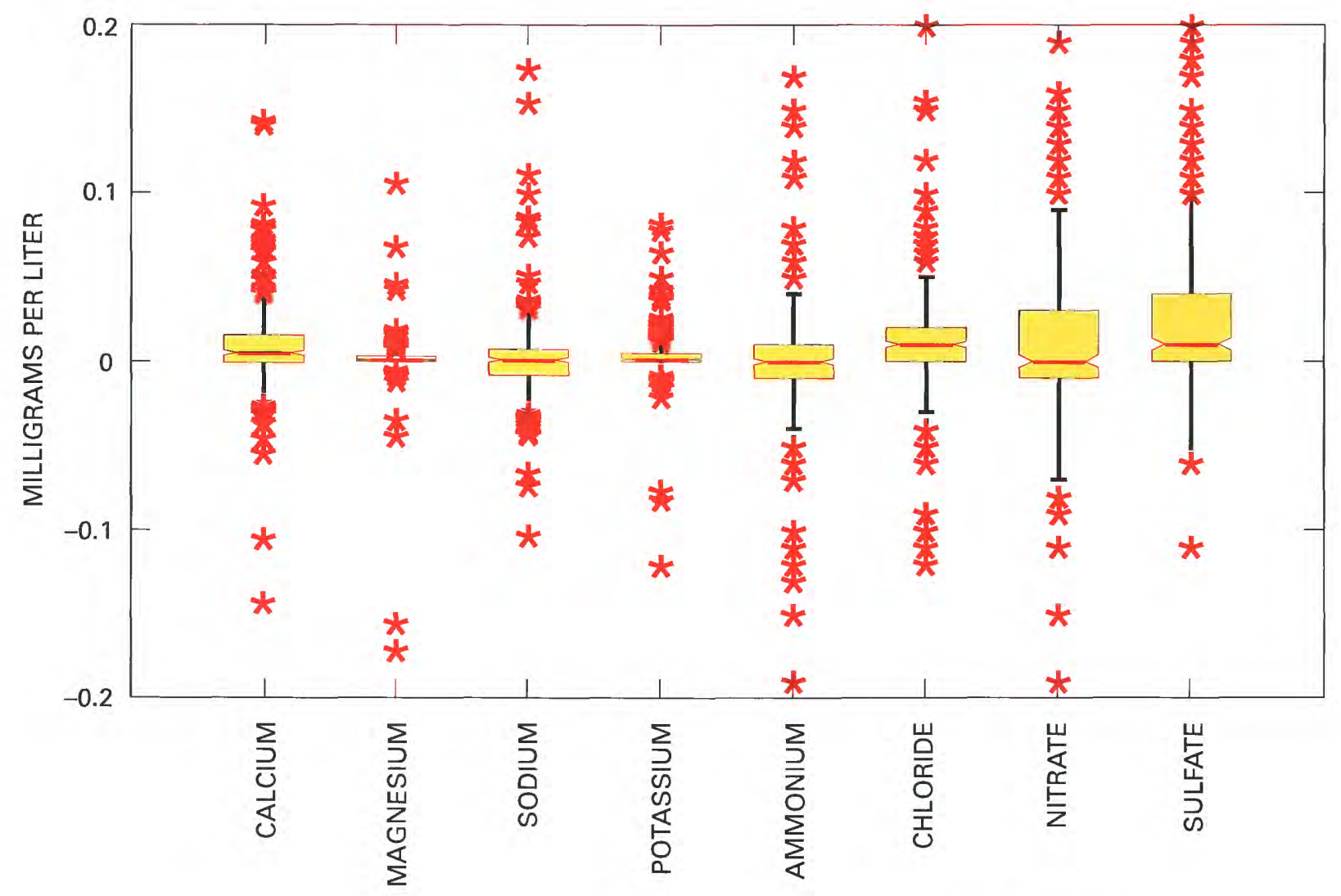

EXPLANATION
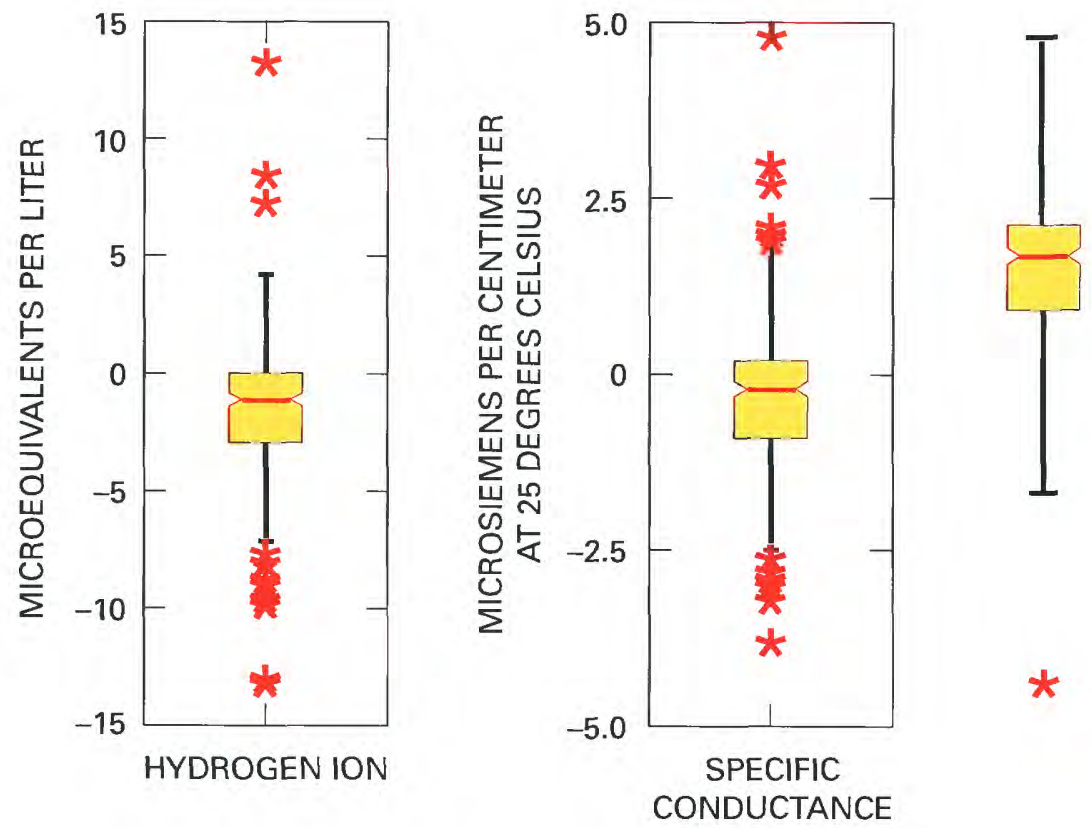

- Largest value less than or equal to the 75 th percentile plus 1.5 times the interquartile range

Upper quartile

Median

Lower quartile

- Smallest value less than or equal to the 25 th percentile minus 1.5 times the interquartile range

Remaining 1 percent of the data not inclusive in the distribution tails

Figure 8. Paired bucket-sample concentrations minus bottle-sample concentrations in the blind-audit program during 1997 through 1999. 


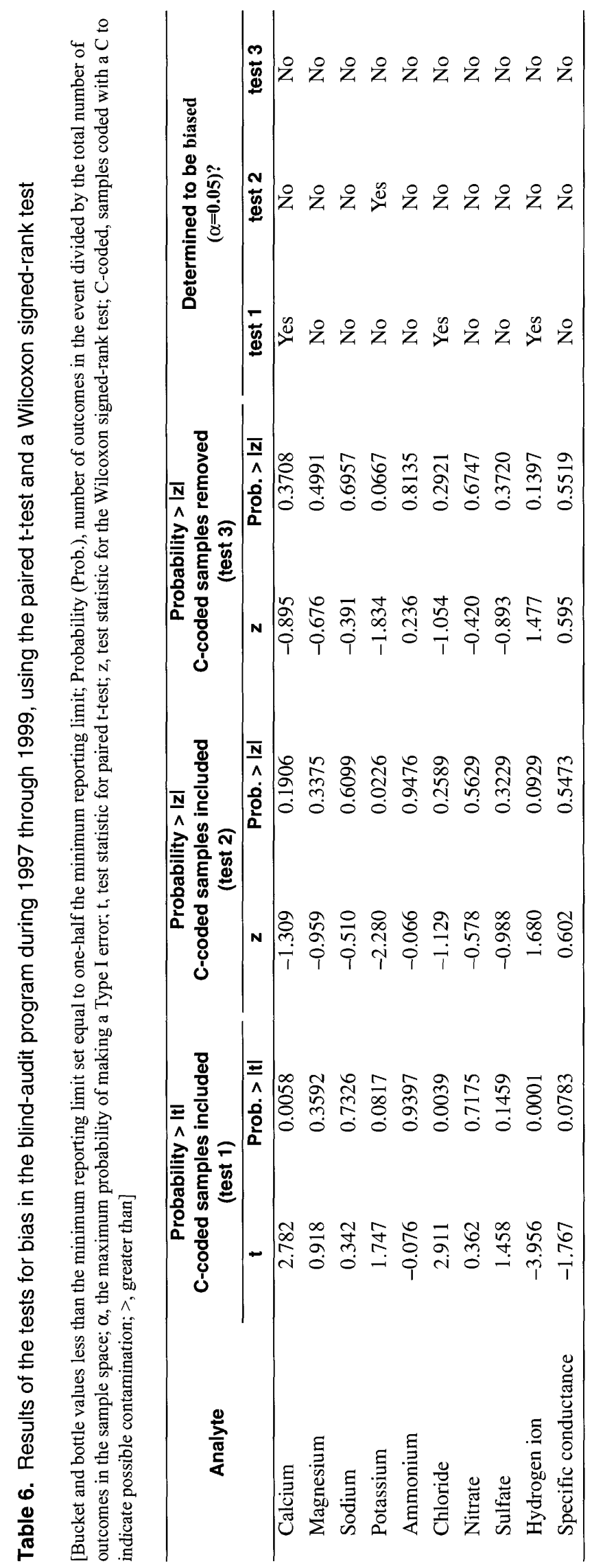


$0.000 \mathrm{mg} / \mathrm{L}$ for nitrate and ammonium to $0.010 \mathrm{mg} / \mathrm{L}$ for sulfate and chloride. The overall median paired differences from all blind-audit samples for hydrogen ion and specific conductance were $-1.086 \mu \mathrm{eq} / \mathrm{L}$ and $-0.200 \mu \mathrm{S} / \mathrm{cm}$, respectively, during 1997 through 1999 (table 5). The interquartile ranges for all of the analytes during 1997 through 1999 were generally smaller than in 1995-96 (Gordon, 1999) and ranged from $0.003 \mathrm{mg} / \mathrm{L}$ for magnesium to $0.04 \mathrm{mg} / \mathrm{L}$ for nitrate and sulfate. For some of the analytes, the interquartile range decreased by 70 percent or more compared to the 1995-96 time period. The improved precision found in the 1997-99 blind-audit results compared to previous years indicates that shipping, handling, and processing had little effect on the blind-audit samples. By inference, the weekly NADP/NTN precipitation samples were equally unaffected by routine shipping, handling, and processing procedures during 1997 through 1999.

\section{Precision}

Precision was evaluated by graphical presentation in boxplots and by statistical analysis of variance, and absolute percent differences. Boxplots in figure 9 depict paired blind-audit differences by sample concentration. The paired blind-audit differences for hydrogen ion and specific conductance, arranged by sample concentration, are shown in figure 10. As in 1995-96, a relation between sample concentration and paired blind-audit differences is not obvious for most analytes upon visual inspection of these graphs. Figures 9 and 10 illustrate two important points: the data in these figures cluster around zero and display non-constant variance or heteroscedasticity (Helsel and Hirsch, 1992).

Results of a Kruskal-Wallis analysis of variance test indicate a statistically significant relation between the magnitude of paired blind-audit differences and sample concentration for sodium, potassium, ammonium, chloride, sulfate, hydrogen ion, and specific conductance in 1997. In 1998, statistically significant relations between the magnitude of paired blind-audit differences and sample concentration were found only for sulfate and specific conductance. In 1999, statistically significant relations between the magnitude of paired blind-audit differences and sample concentration were found for magnesium, sodium, chloride, nitrate, sulfate, hydrogen ion, and specific conductance (table 7). The importance placed on these findings must be tempered by the departure from equal variances (heteroscedasticity) observed in the data. Heteroscedasticity diminishes the weight one can place on the results of the Kruskal-Wallis test, although as in previous years, the departure from equal variances was not large enough to nullify the results (Gordon, 1999). The implication from this analysis is that the larger the concentration in a blind-audit sample, the greater the magnitude of the paired blind-audit sample differences for these analytes. By inference, it is expected that the same relation between analyte concentration and variance holds true for weekly NADP/NTN precipitation samples. Other researchers have confirmed similar relations between analyte concentration and variance (Miller, 1991).

Relative and absolute percent differences were calculated for all of the paired blind-audit samples. Relative percent differences are useful for understanding bias (assuming the distribution of relative and absolute percent differences is taken into consideration), whereas absolute percent differences are useful for understanding variability (precision). The upper and lower quartiles as well as the median relative and absolute percent differences during 1997 through 1999 are listed in table 8. The relative and absolute percent differences were determined by calculating each paired blind-audit difference as a percentage of the known sample concentration:

$$
\begin{gathered}
\text { Relative percent difference }= \\
{\left[\left(\mathrm{C}_{1}-\mathrm{C}_{2}\right) / \mathrm{C}_{3}\right] \times 100} \\
\text { and }
\end{gathered}
$$

$$
\begin{gathered}
\text { Absolute percent difference }= \\
\qquad\left|\left(\mathrm{C}_{1}-\mathrm{C}_{2}\right) / \mathrm{C}_{3}\right| \times 100
\end{gathered}
$$

where

$$
\begin{array}{r}
\mathrm{C}_{1}=\begin{array}{c}
\text { sample concentration, in milligrams per liter, } \\
\text { from the portion of the blind-audit sample } \\
\text { exposed to all handling and processing steps } \\
\text { of a normal weekly precipitation sample; }
\end{array} \\
\mathrm{C}_{2}=\text { sample concentration, in milligrams per liter, } \\
\text { from the control portion of the blind-audit } \\
\text { sample subject to minimal handling and } \\
\text { processing; and }
\end{array}
$$

Bucket-bottle data pairs were excluded for a given analyte if the target concentration was less than or equal to the MRL. The percent differences are inflated by the influence of large bucket-bottle differences when the target concentration was less than or equal to the MRL (Nilles and others, 1995). 

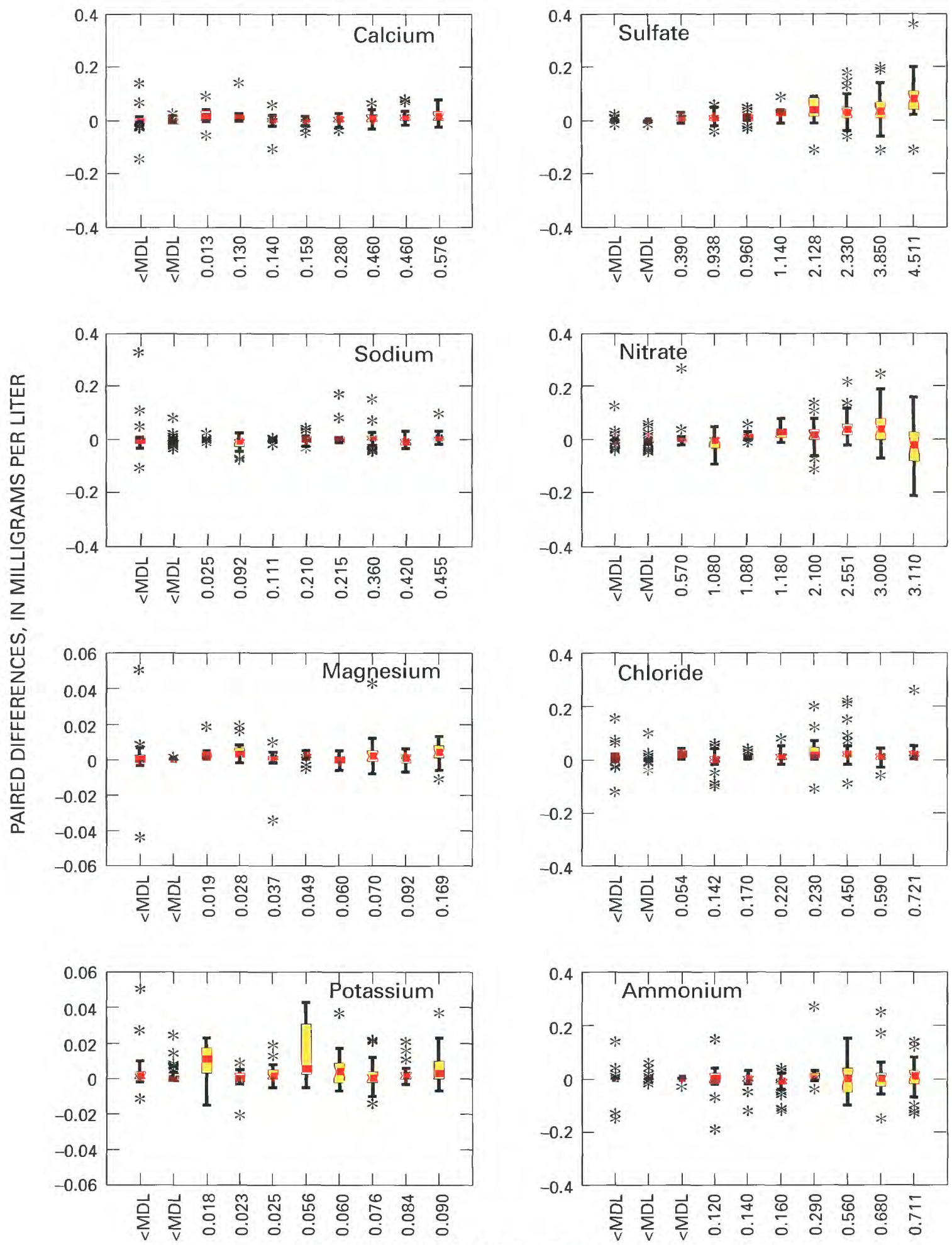

TARGET CONCENTRATION, IN MILLIGRAMS PER LITER

\section{EXPLANATION}

$<$ MDL Target less than method detection limit

* Remaining 1 percent of data not inclusive in the distribution tails

Figure 9. Relation between paired blind-audit differences for major ions and the analyte concentrations of solutions used in the blind-audit program during 1997 through 1999. 

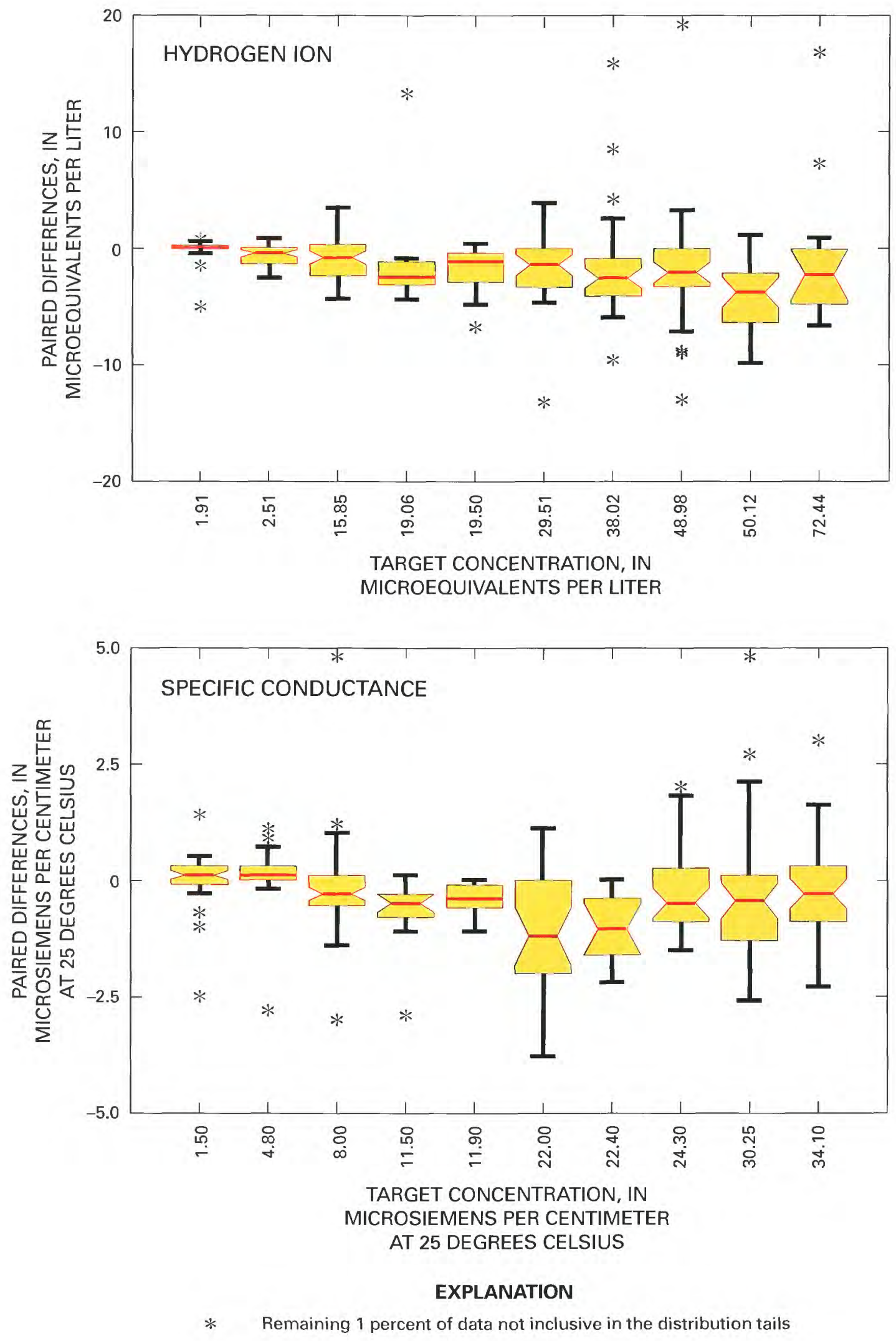

Figure 10. Relation between paired blind-audit differences for hydrogen ion and specific conductance and the analyte concentrations of solutions used in the blind-audit program during 1997 through 1999. 
Table 7. Results of the Kruskal-Wallis analysis of variance test to determine the relation between paired blind-audit sample differences and the target concentrations used in the blind-audit program in 1997, 1998, and 1999

[All paired blind-audit differences expressed in milligrams per lite, except specific conductance in microsiemens per centimeter at 25 degrees Celsius and hydrogen ion in microequivalents per liter; $<$, less than]

\begin{tabular}{|c|c|c|c|c|c|c|}
\hline \multirow[t]{2}{*}{ Analyte } & \multicolumn{3}{|c|}{$\begin{array}{l}\text { Bucket minus bottle differences } \\
\text { in significance levels ( } p \text {-values) } \\
\text { by target concentrations }\end{array}$} & \multicolumn{3}{|c|}{$\begin{array}{l}\text { Statistically significant } \\
\qquad(\alpha=0.05) ?\end{array}$} \\
\hline & 1997 & 1998 & 1999 & 1997 & 1998 & 1999 \\
\hline Magnesium & 0.5407 & 0.5985 & 0.0004 & No & No & Yes \\
\hline Sodium & 0.0084 & 0.1797 & 0.0061 & Yes & No & Yes \\
\hline Ammonium & 0.0245 & 0.6592 & 0.8451 & Yes & No & No \\
\hline Chloride & 0.0004 & 0.0543 & $<0.001$ & Yes & No & Yes \\
\hline Nitrate & 0.1345 & 0.0506 & $<0.001$ & No & No & Yes \\
\hline Sulfate & 0.0102 & 0.0019 & $<0.001$ & Yes & Yes & Yes \\
\hline Hydrogen ion & 0.0007 & 0.1463 & 0.0019 & Yes & No & Yes \\
\hline
\end{tabular}

Table 8. Relative and absolute bucket minus bottle differences calculated as a percentage of the target bottle concentration for each analyte during 1997 through 1999 for the blind-audit program

\begin{tabular}{|c|c|c|c|c|c|c|}
\hline \multirow[t]{3}{*}{ Analyte } & \multirow{2}{*}{\multicolumn{3}{|c|}{$\begin{array}{c}\text { Relative bucket minus bottle differences, } \\
\text { expressed as a percentage of corresponding } \\
\text { target bottle concentration } \\
\text { (selected data pairs only) }\end{array}$}} & \multicolumn{3}{|c|}{$\begin{array}{l}\text { Absolute bucket minus bottle differences, } \\
\text { expressed as a percentage of corresponding } \\
\text { target bottle concentration } \\
\text { (selected data pairs only) }\end{array}$} \\
\hline & & & & \multicolumn{3}{|c|}{ Percentiles } \\
\hline & 25th & 50th & 75th & 25th & 50th & 75th \\
\hline Calcium & -0.71 & 2.63 & 6.45 & 1.91 & 3.70 & 7.86 \\
\hline Magnesium & 0.00 & 2.22 & 5.41 & 1.79 & 3.26 & 6.67 \\
\hline Sodium & -3.10 & 0.90 & 3.33 & 1.43 & 3.33 & 7.62 \\
\hline Potassium & 0.00 & 4.35 & 13.25 & 2.63 & 7.14 & 15.00 \\
\hline Ammonium & -6.25 & 0.00 & 2.82 & 0.00 & 4.41 & 10.71 \\
\hline Chloride & 0.00 & 2.78 & 5.88 & 1.39 & 4.44 & 8.70 \\
\hline Nitrate & 0.00 & 0.93 & 1.96 & 0.78 & 1.67 & 2.94 \\
\hline Sulfate & 0.43 & 1.29 & 2.56 & 0.89 & 1.72 & 2.56 \\
\hline Hydrogen ion & -11.77 & -4.50 & 1.94 & 3.50 & 6.91 & 14.48 \\
\hline Specific conductance & -4.65 & -1.87 & 0.93 & 1.32 & 3.52 & 6.25 \\
\hline
\end{tabular}


During 1997-99, the median (50th percentile) relative percent differences for all analytes was within a narrow range of -4.50 to +4.35 percent, indicating minor positive or negative bias (table 8). These results contrast with results from 1995-96, when ammonium had a median relative bias of -9.72 percent in 1995 and -12.50 percent in 1996 , and the median relative percent bias for sodium was -7.99 percent in 1995 and -7.00 percent in 1996 . Chloride also showed a high median relative percent bias of -7.04 percent in 1996 .

During 1997-99, the median absolute differences in percent did not exceed 8 percent for any of the analytes. This is in contrast with the results from 1995-96, when in addition to negative biases for sodium and ammonium, the median absolute difference values for ammonium and sodium ranged from 11.13 to 14.29 percent. During 1997-99, the median absolute difference was 3.33 percent for sodium and 4.41 percent for ammonium (table 8).

Three sample volumes were used in the blindaudit program to investigate a possible relation between sample volume collected weekly at NADP/NTN sites and the amount of contamination introduced through shipping and handling procedures. Differences between bucket and bottle pairs were analyzed in their original concentration units and in units of mass. Boxplots in figures 11 and 12 show the differences for each analyte plotted by sample volume in units of mass.

To determine if there was a statistically significant relation between paired blind-audit differences and sample volume, a Kruskal-Wallis test was performed. An equal number of samples containing 250, 500, or $1,000 \mathrm{~mL}$ of solution were included in each quarterly mailing of samples throughout 1997-99. Kruskal-Wallis results for 1997-99 indicate a significant $(\alpha=0.05)$ relation between paired blind-audit sample differences in units of concentration and sample volume for magnesium, chloride, sulfate, and hydrogen ion (table 9). Before the January 1994 protocol change, each year at least 5 of the 10 measured analytes displayed a statistically significant relation between paired blind-audit differences in units of concentration and sample volume. When paired differences were converted to units of mass, the outcome of the Kruskal-Wallis test was different: statistically significant $(\alpha=0.05)$ differences during 1997 through 1999 between the bucket and bottle pairs were found for magnesium and sulfate. The boxplots in figures 11 and 12 show that bucket-bottle differences in units of mass were generally centered on the zero difference line for the 1997-99 timeframe, in contrast with the results from 1994-96 when paired differences in units of mass tended to become more negative with increasing sample volume (Gordon, 1999; Gordon and others, 1997). Before 1994, paired bucket minus bottle differences in units of mass were generally positive and increased with increasing sample volume (Nilles and others, 1993).

Figures 13 through 16 show locally weighted scatterplot smoothing (LOWESS) that was used to depict trends in the paired bucket minus bottle blindaudit results during 1997 through 1999. The LOWESS smoothing method described by Cleveland (1985) is a powerful tool for depicting trends in the blind-audit results over time. The LOWESS method does not assume a functional relation between concentration and time; nonlinearities in trends can readily be observed (Lynch and others, 1996). LOWESS lines were drawn through approximately the upper and lower quartiles of the distribution for each analyte (75th and 25th percentiles) as well as through the median of the distribution. Key features of the data distribution for each analyte are thus depicted as they vary or remain relatively constant over time. The LOWESS smooth lines for chloride, ammonium, potassium, magnesium, and nitrate (figs. 13,14, and 15, respectively) are fairly consistent throughout the 3 years. The LOWESS smooth lines for sulfate diverge slightly during 1999 as slightly more variability in paired blind-audit results occurred (fig. 13). The LOWESS smooth lines for sodium (fig. 16) nearly converge in late 1998 and remained in a narrow range during 1999. Upon arrival at the CAL, both the bucket and the bottle portions are filtered. In late 1997, the CAL's supply of filters was depleted, and the filters used for filtering the NADP/NTN samples were switched from cellulose ester membrane (HAWP 04700, 0.45- $\mu \mathrm{m}$, Millipore Corporation) filters to Gelman Supor ${ }^{\circledR} 450,0.45-\mu \mathrm{m}$ polyethersulfone filters. In October 1998, John Gordon of the USGS and Jane Rothert of the Illinois State Water Survey discussed the results of the blind-audit program. Together, they traced the probable source of sodium contamination to the Millipore filters used until November 1997 to filter samples before laboratory analysis. Bucket-sample portions with larger volumes had more water available for rinsing the filter (limited by filtering apparatus capacity, about $200 \mathrm{~mL}$ ) before collecting the final aliquot for analysis and therefore were less contaminated than samples with smaller volumes available for rinsing. 

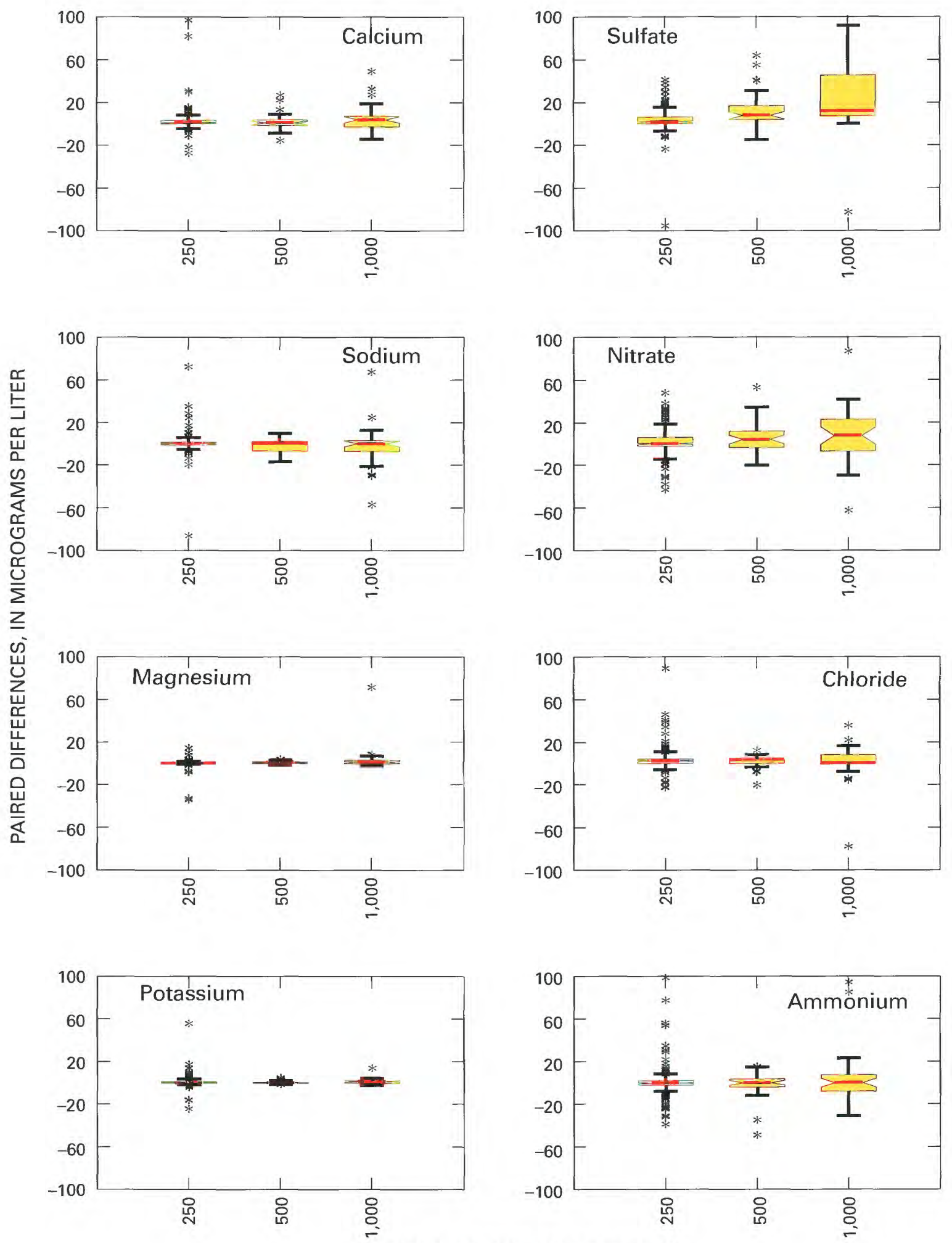

SAMPLE VOLUME, IN MILLILITERS

\section{EXPLANATION}

* Remaining 1 percent of data not inclusive in the distribution tails

Figure 11. Relation between paired blind-audit differences for the major ions and sample volume for the 250-, 500-, and 1,000-milliliter U.S. Geological Survey solution samples during 1997 through 1999. 

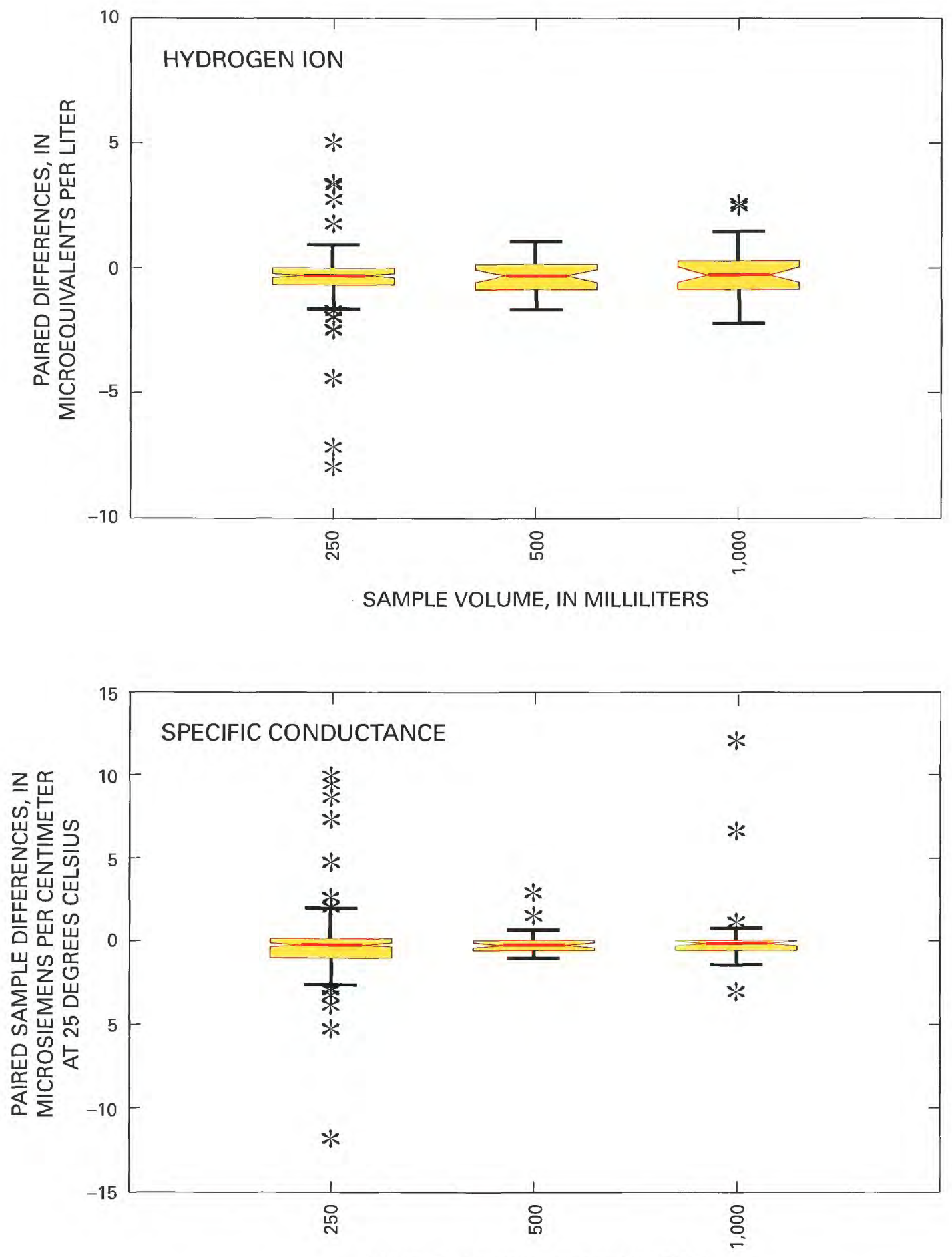

SAMPLE VOLUME, IN MILLILITERS

\section{EXPLANATION}

* Remaining 1 percent of data not inclusive in the distribution tails

Figure 12. Relation between paired blind-audit differences for hydrogen ion and specific conductance and sample volume for the 250-, 500-, and 1,000-milliliter U.S. Geological Survey solution samples during 1997 through 1999. 
Table 9. Results of the 1997 through 1999 Kruskal-Wallis analysis of variance tests to determine if bucket minus bottle differences for the 250-, 500-, and 1,000-milliliter samples of the USGS solution used in the blind-audit program have equivalent distributions

[mL, milliliter; USGS, U.S. Geological Survey; <, less than]

\begin{tabular}{|c|c|c|c|c|}
\hline Analyte & $\begin{array}{l}\text { Attained significance } \\
\text { ( } p \text {-value) levels of } \\
\text { bucket minus bottle } \\
\text { differences on a mass } \\
\text { per bucket basis }\end{array}$ & $\begin{array}{c}\text { Statistically significant } \\
(\alpha=0.05) \text { differences } \\
\text { determined between } 250-, \\
500-, \text { and } 1,000-m L \text { USGS } \\
\text { samples on a mass per } \\
\text { bucket basis? }\end{array}$ & $\begin{array}{l}\text { Attained significance } \\
\text { ( } p \text {-value) levels of } \\
\text { bucket minus bottle } \\
\text { differences on a } \\
\text { concentration basis }\end{array}$ & $\begin{array}{c}\text { Statistically significant } \\
(\alpha=0.05) \text { differences } \\
\text { determined between } 250-, 500- \\
\text { and } 1,000-m L \text { USGS samples } \\
\text { on a concentration basis? }\end{array}$ \\
\hline Calcium & 0.2599 & No & 0.3084 & No \\
\hline Magnesium & $<0.0001$ & Yes & 0.0204 & Yes \\
\hline Sodium & 0.6697 & No & 0.3036 & No \\
\hline Potassium & 0.0826 & No & 0.1976 & No \\
\hline Ammonium & 0.7512 & No & 0.8181 & No \\
\hline Chloride & 0.7667 & No & 0.0203 & Yes \\
\hline Nitrate & 0.0575 & No & 0.6256 & No \\
\hline Sulfate & $<0.0001$ & Yes & 0.0027 & Yes \\
\hline Hydrogen ion & 0.8609 & No & 0.0012 & Yes \\
\hline Specific conductance & 0.8500 & No & 0.3280 & No \\
\hline
\end{tabular}

\section{Ultrapure Deionized-Water Sample Results}

Sixteen Ultrapure deionized-water samples were included in the blind-audit program in 1997 and in 1999; in 1998 there were 14 Ultrapure blind-audit samples. All of the Ultrapure samples were submitted correctly each year, resulting in complete data pairs. As with all blind-audit samples, deionized-water samples were processed in two parts: the first part, the bucket portion, was exposed to normal sample-handling steps, representing the field-exposed portion, and the second part, the bottle portion, was subject to minimal handling, representing the control portion. In all 16 of the deionized-water samples submitted in 1997, sodium was detected in the field-exposed portion; in the control portion, sodium was detected in 14 of the 16 samples. Ammonium, chloride, and nitrate were detected at least three times in both sample portions (table 10). In 1998, the incidence of analyte detection in the Ultrapure samples was much lower than in 1997. Only calcium was detected more than twice in the bottle portion of the Ultrapure samples, albeit the total number of Ultrapure samples was 14 in 1998 compared to 16 in 1997. In 1999 , the incidence of analyte detection in the bottle portion of the Ultrapure samples was again lower than in 1997 (except calcium, magnesium, and sulfate). However, the 1999 incidences of values exceeding the reporting limits were slightly higher on average than the 1998 incidences (table 10).

\section{FIELD-AUDIT PROGRAM}

The field-audit program was designed to evaluate the effects of field exposure, handling, and processing on the chemistry of NADP/NTN precipitation samples. The NADP/NTN Network Operations Subcommittee approved the concept of a field-audit program in April 1996. As the newest external qualityassurance program for the NADP, the field-audit program was designed as a cost-effective way to measure how field exposure of sample-collection equipment affects precipitation chemistry. This program replaced the weekly, network-wide analysis of the dry wet-side buckets. The field-audit program operated as a pilot study between August 1996 and March 1997. During this time the protocols were developed and tested, and preparation was made for widespread implementation. Since July 1997, fieldaudit samples have been distributed to 25 NADP/NTN sites on a quarterly basis. Figure 17 outlines the components of the field-audit program, from sample preparation to distribution of interpretive reports.

In the field-audit program, the site operator is instructed to process and submit a quality-control sample following a standard 7-day, Tuesday-to-Tuesday sampling period with no precipitation. The requirement of a full week without precipitation prevents loss of the routine weekly precipitation-chemistry data collected at the site. A number of prerequisite conditions must be met before proceeding with sample collection. 

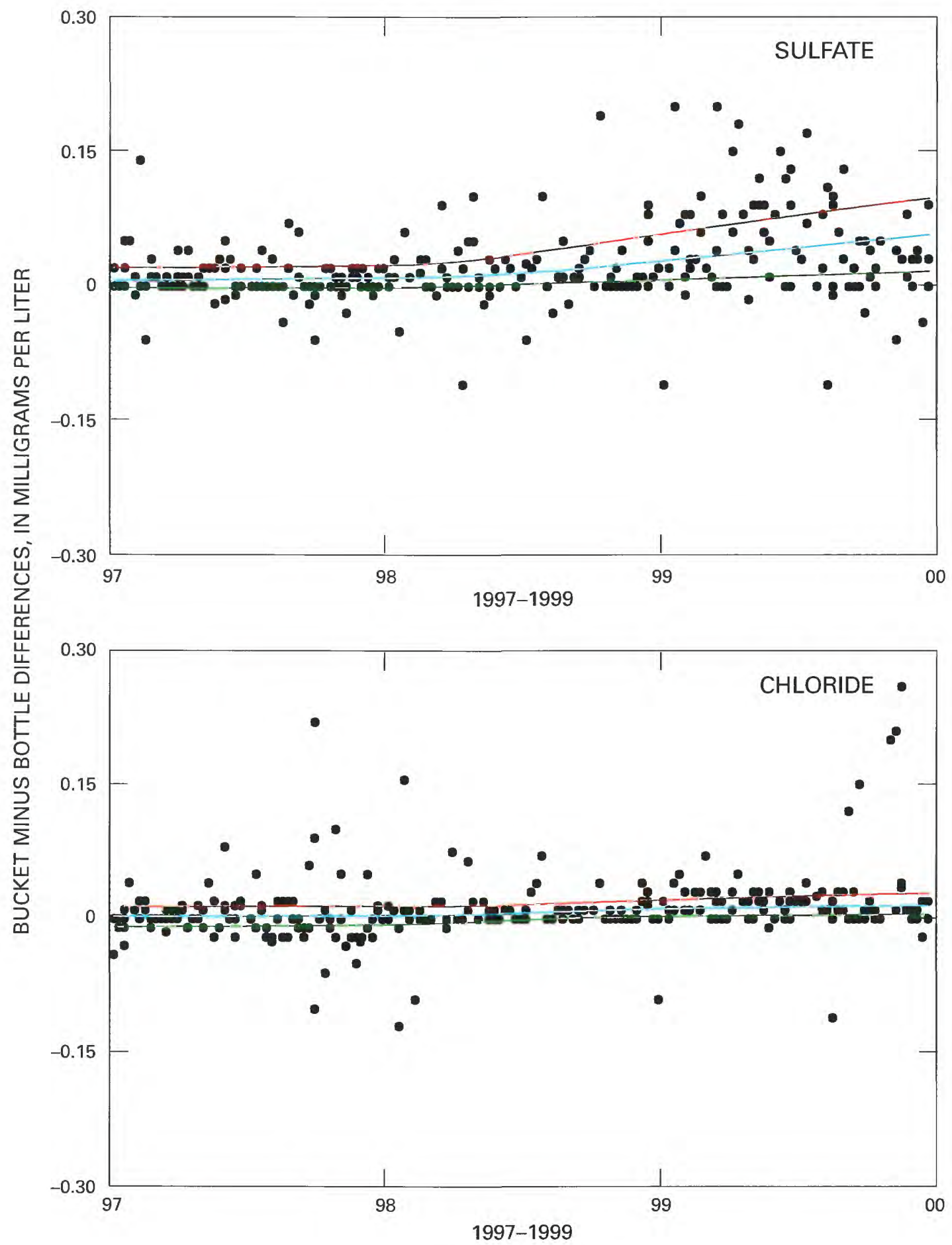

\section{EXPLANATION}

75th percentile LOWESS smooth

LOWESS, Locally Weighted Scatterplot Smoothing Median LOWESS smooth

25th percentile LOWESS smooth

Figure 13. Locally weighted scatterplot smoothing for the blind-audit program for the analytes sulfate and chloride during 1997 through 1999. 


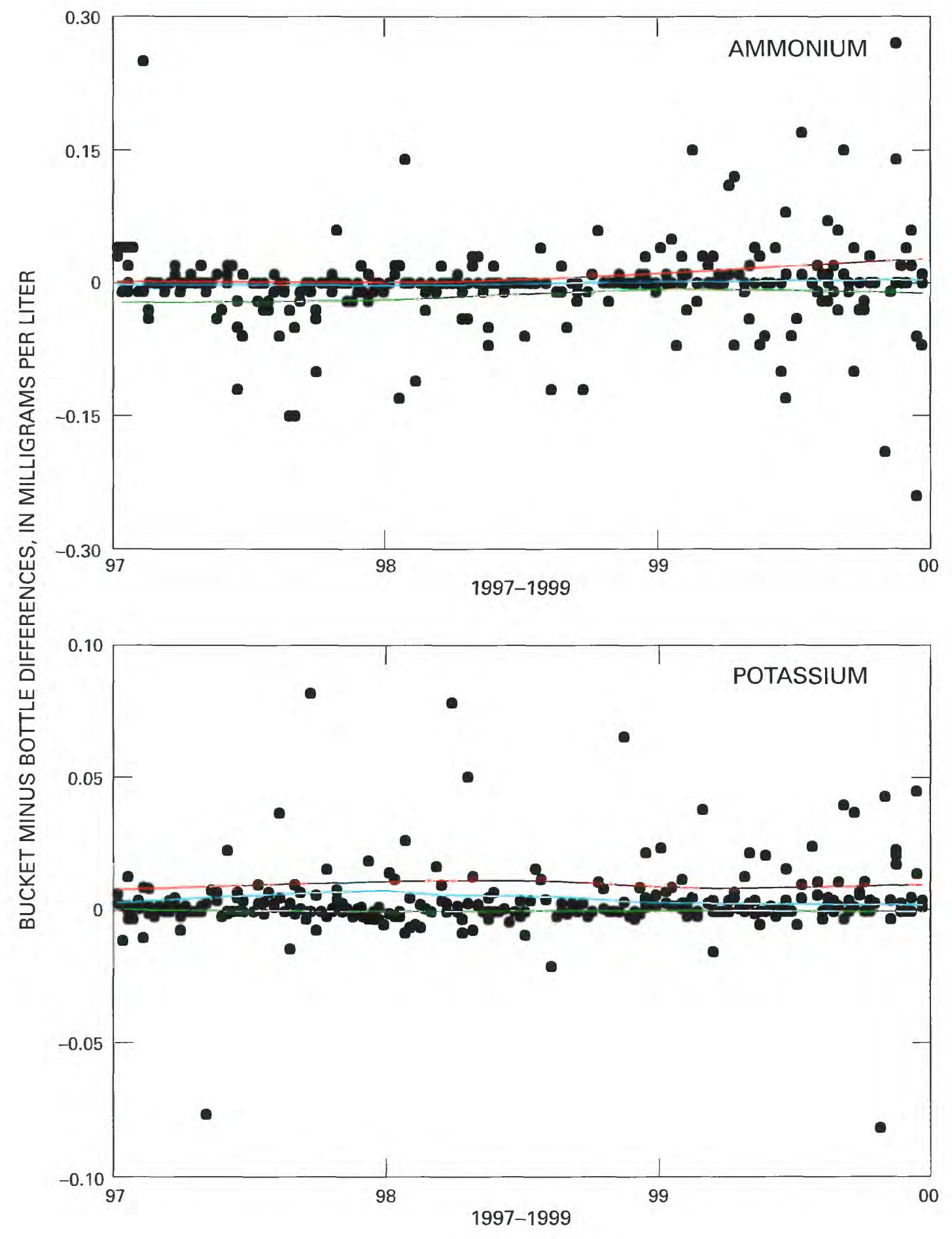

\section{EXPLANATION}

75th percentile LOWESS smooth LOWESS, Locally Weighted Scatterplot Smoothing Median LOWESS smooth 25th percentile LOWESS smooth

Figure 14. Locally weighted scatterplot smoothing for the blind-audit program for the analytes ammonium and potassium during 1997 through 1999. 

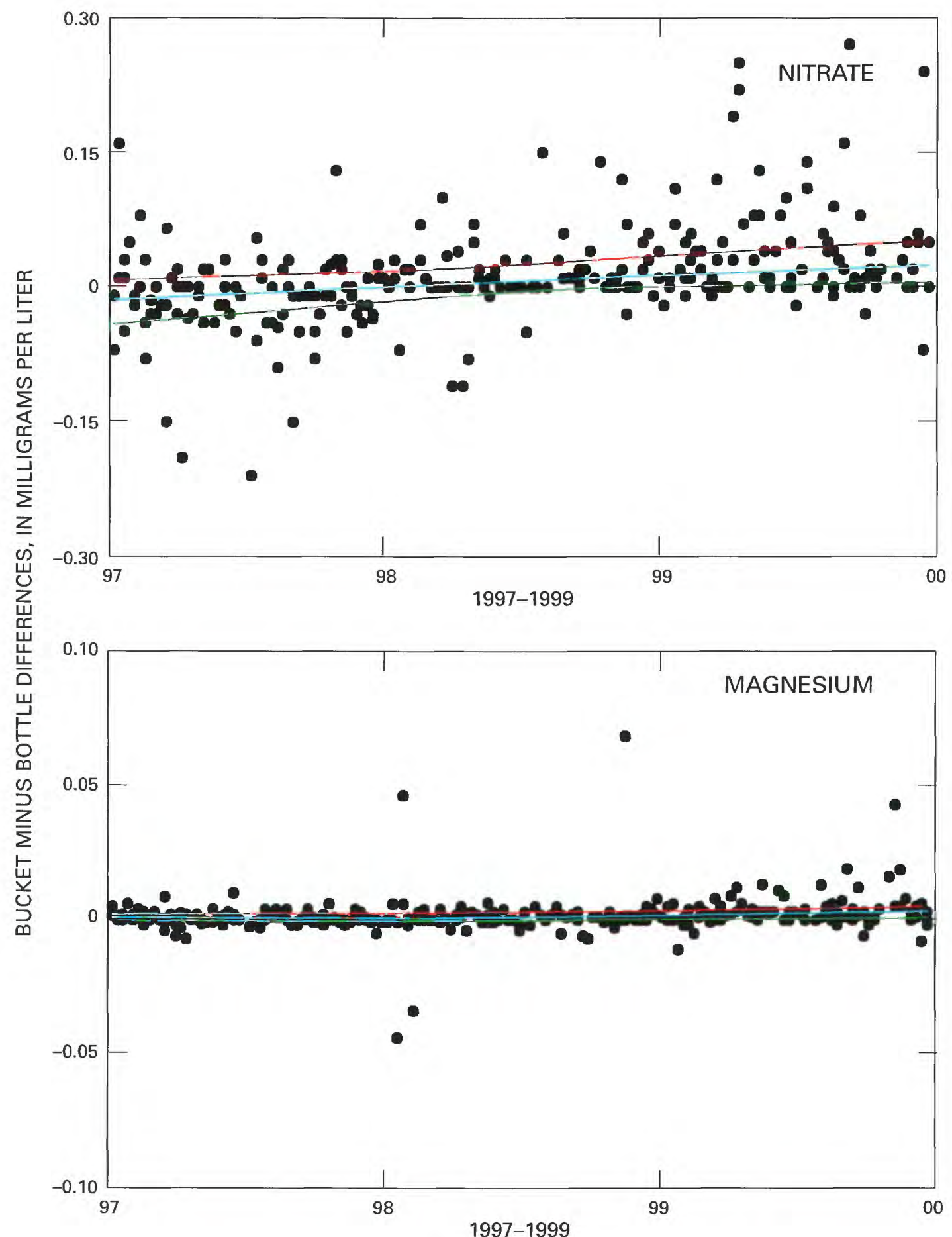

\section{EXPLANATION}

75th percentile LOWESS smooth

LOWESS, Locally Weighted Scatterplot Smoothing Median LOWESS smooth 25th percentile LOWESS smooth

Figure 15. Locally weighted scatterplot smoothing for the blind-audit program for the analytes nitrate and magnesium during 1997 through 1999. 


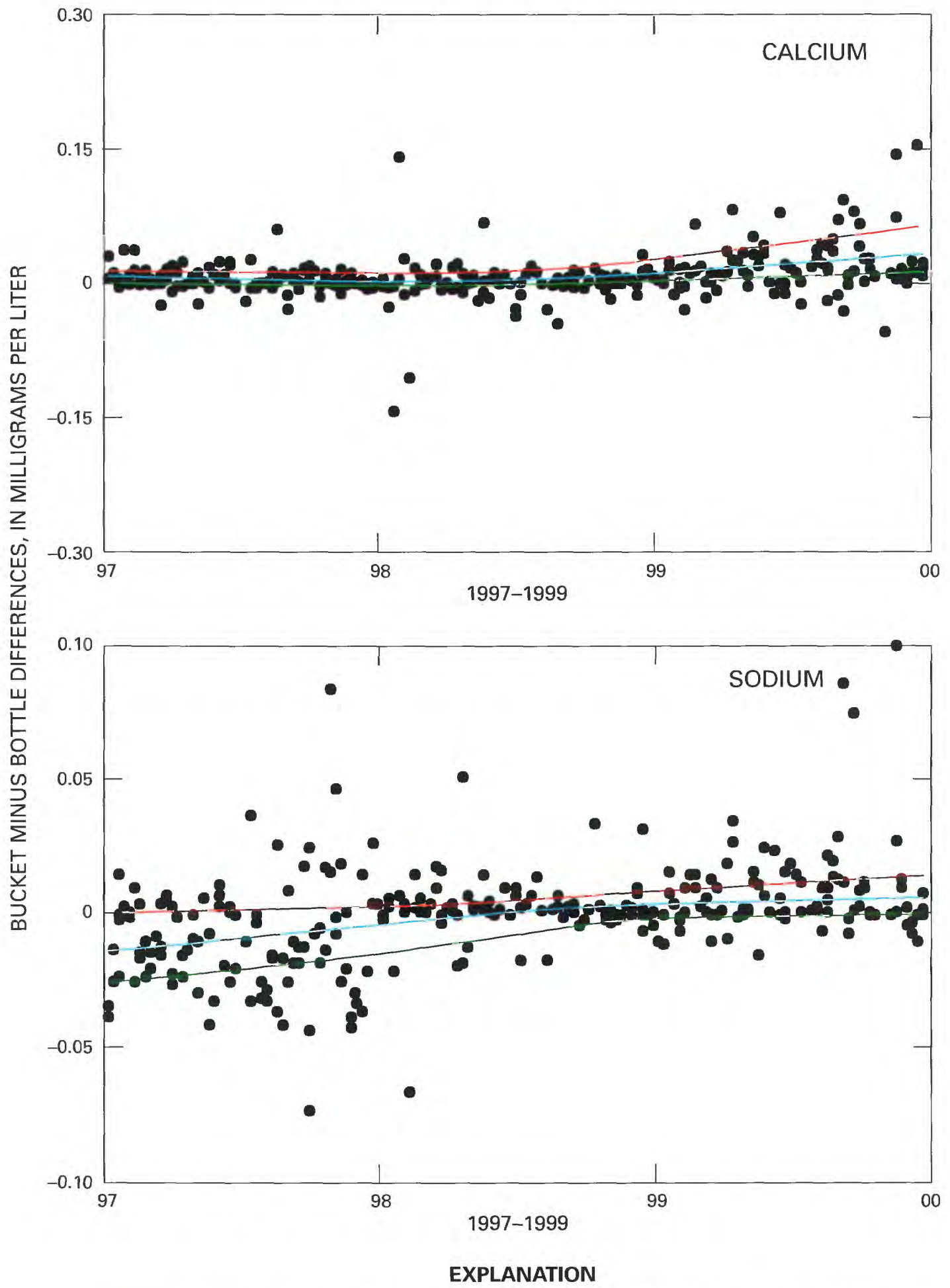

75th percentile LOWESS smooth

LOWESS, Locally Weighted Scatterplot Smoothing Median LOWESS smooth

25th percentile LOWESS smooth

Figure 16. Locally weighted scatterplot smoothing for the blind-audit program for the analytes calcium and sodium during 1997 through 1999. 
Table 10. Number of determinations exceeding the minimum reporting limit for the Ultrapure deionized-water samples submitted annually as part of the blind-audit program in 1997, 1998, and 1999

\begin{tabular}{lrrrrrr}
\hline \multirow{2}{*}{ Analyte } & \multicolumn{3}{c}{$\begin{array}{c}\text { Field-exposed } \\
\text { bucket portion }\end{array}$} & \multicolumn{3}{c}{$\begin{array}{c}\text { Minimally handled } \\
\text { bottle portion }\end{array}$} \\
\cline { 2 - 7 } & $\mathbf{1 9 9 7}$ & $\mathbf{1 9 9 8}$ & $\mathbf{1 9 9 9}$ & $\mathbf{1 9 9 7}$ & $\mathbf{1 9 9 8}$ & $\mathbf{1 9 9 9}$ \\
\hline Calcium & 6 & 7 & 11 & 2 & 5 & 4 \\
Magnesium & 1 & 0 & 3 & 0 & 0 & 1 \\
Sodium & 16 & 8 & 5 & 14 & 2 & 3 \\
Potassium & 6 & 4 & 5 & 1 & 0 & 1 \\
Ammonium & 6 & 0 & 2 & 3 & 0 & 1 \\
Chloride & 10 & 1 & 1 & 6 & 0 & 1 \\
Nitrate & 15 & 1 & 0 & 14 & 0 & 1 \\
Sulfate & 0 & 0 & 0 & 0 & 0 & 2 \\
\hline
\end{tabular}

The site operator must check the rain-gage chart to make sure the Aerochem Metrics collector lid did not open and uncover the wet-side bucket during the sampling period. This is verified by checking the event pen to ensure it did not record any marks indicating that the sensor was activated. If the site is located in an area with extremely high humidity, the probability of a week with no lid openings is very low. The site operator is furnished with special instructions to accommodate the collection of field-audit data from these sites. Finally, the site operator inspects the wet-side bucket to ensure that it is at least as dry as it was when it was installed the previous week. "Wet" and "dry" samples are used in the field-audit program. If there were a few drops of rinse water in the bucket when it was installed, it is conceivable that the water is still present. A bucket is considered "wet" if there was CAL rinse water in the bucket when the bucket was installed, and if the rinse water remains at the end of the week, with no additional deposition. Rain or dryfall would not have had an opportunity to enter the bucket because the lid was never in the open position. The term "dry" is defined as no rinse or rainwater present. If all of the requirements are met, the operator pours 75 percent of the supplied sample into the bucket, swirls the sample in the bucket, and then provides for a residence time of a least 24 hours before transferring the sample to a clean 1- $\mathrm{L}$ shipping bottle. This sample is processed by the CAL as if it were an actual precipitation sample from the site. The 25 percent of the sample portion remaining in the original sample container is shipped to the CAL for separate analysis.
Unlike the blind-audit program, field-audit samples are not disguised as environmental samples. Because there is no prespecified sample submission date, the site operator contacts the CAL NADP/NTN site liaison immediately after the sample is submitted. The site operator has a full year from when their original quarter ends to meet the conditions specified by the program and submit their sample. The site operator fills out postcards notifying the USGS and the Program Office that the sample was submitted. This notification process helps ensure that the data are correctly coded in the database.

Like the blind-audit program, the field-audit program utilizes a paired sample design to increase the statistical power for detecting differences. Unlike the blind-audit program, which is designed to measure the effects of routine shipping, handling, and processing on sample chemistry, the purpose of the field-audit program is to quantify precipitation-chemistry changes resulting from normal field exposure of the samplecollection container. During normal operations of the NADP/NTN sites, every Tuesday morning at all sites across the network, the sample from the previous week is removed and a new sample-collection bucket is installed in the Aerochem Metrics wet-deposition collector. Although the bucket is covered with a foam pad attached to a rigid piece of aluminum when precipitation is not occurring, small amounts of contamination can enter the bucket. For example, small amounts of windblown dust can enter the bucket, particularly when the foam lid pad has started to wear and the seal between the bucket and lid deteriorates. Small amounts of dust or debris can fall into the bucket when the lid is in motion. The net effect of these influences can change the chemistry of the precipitation sample.

\section{Data Analysis}

Forty of the 75 field-audit samples, mailed between July and December 1997, were submitted for analysis by the end of 1997. Eighty samples were submitted for analysis in 1998. Sixty-three of these were from the set of 100 samples sent to site operators throughout 1998, and 17 were carryover samples from 1997. Seventy samples were submitted for analysis in 1999. Sixty-five of these were part of the 100 samples sent to site operators during 1999, and 5 were carryovers from 1998. Overall, there were 190 complete 


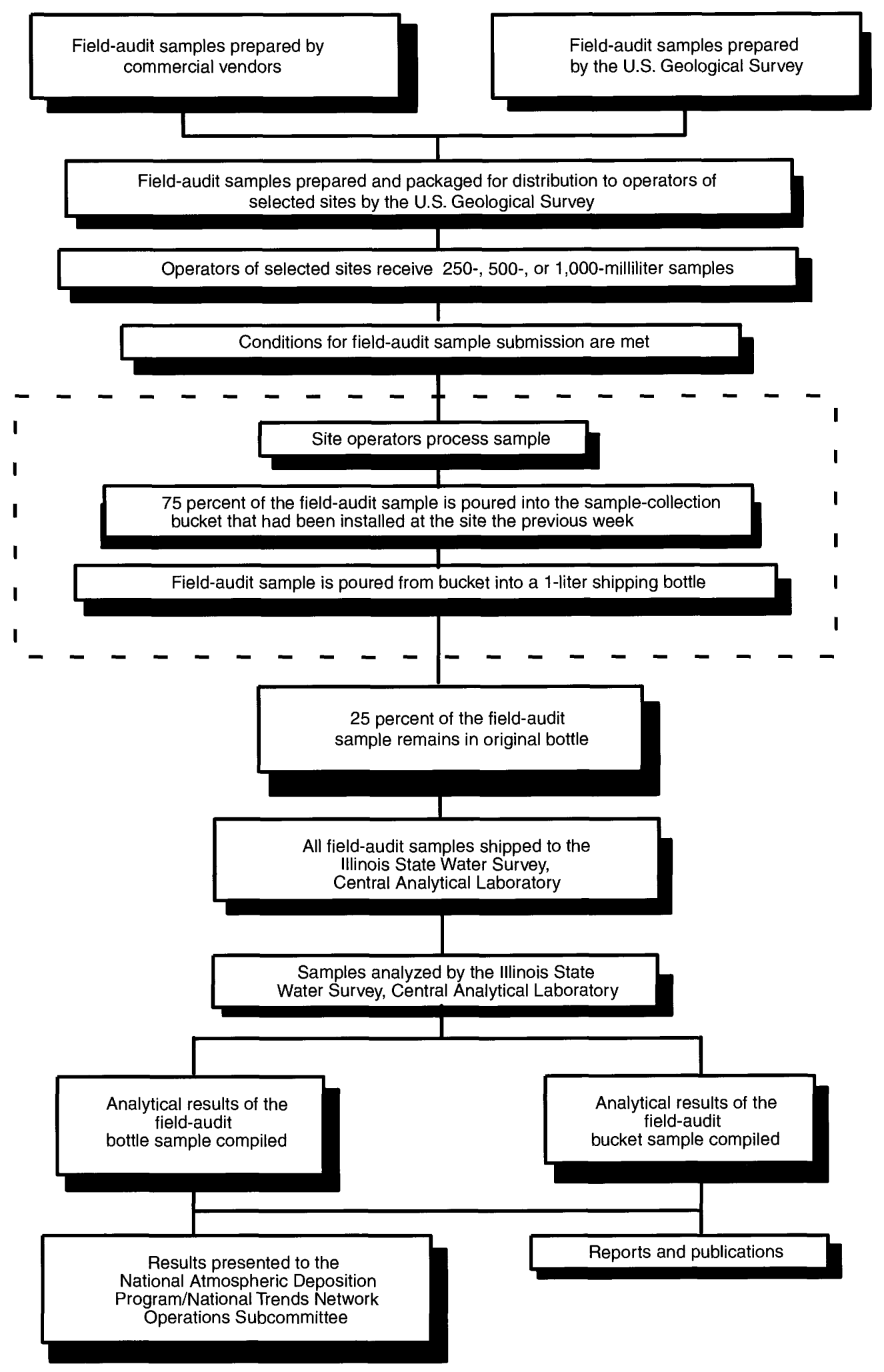

Figure 17. Field-audit program of the U.S. Geological Survey. 
sample pairs for 1997-99. Because of the July 1997 startup and limited number of samples analyzed in 1997, results from 1997-98 are compared with the results from 1999 for this report.

\section{Analytical Bias and Precision}

Samples submitted as part of the field-audit program are referred to as field-audit samples. As with other types of samples, regardless of sample chemistry, bucket and bottle portions of field-audit samples containing extrinsic material are assigned a " $\mathrm{C}$ " code by the CAL. Using the Wilcoxon signed-rank test to compare the two groups of field-audit samples (C-coded samples and samples without " $\mathrm{C}$ " codes) for all major ions, no statistically significant differences in tests for bias were found at the $\alpha=0.05$ level during 1997 through 1999 for any of the analytes (tables 11 and 12). Therefore, data from the field-audit samples assigned "C" codes during 1997 through 1999 were included in the overall statistical analysis of field-audit samples. Before determining paired bucket minus bottle differences for the field-audit program, bucket and bottle values reported as less than the minimum reporting limit (MRL) were set equal to one-half the MRL. Bucket and bottle values less than the MRL were both set equal to the MRL or to zero before the analysis. Because only minor differences resulted from how the "less than" MRL values were handled, all of the "less than" MRL values were set equal to one-half the MRL for the field-audit program.

Since field-audit samples can be put either into a dry bucket or a bucket with rinse water present, the data were initially divided into separate files depending on whether the sample data were coded as "wet" or "dry." Including the 39 C-coded samples, a total of 190 field-audit samples were analyzed during 1997 through 1999. Of the 190 samples analyzed, 102 were processed with rinse water present in the bucket and 88 were processed using dry buckets. Table 13 contains summary statistics for "wet" and "dry" fieldaudit samples. In most cases, the medians of the differences between bucket and bottle portions are very similar for the "wet" coded samples where rinse water was present and the "dry" coded samples where rinse water was absent. The differences are small, and the quartile ranges and medians also are quite similar for the "wet" and "dry" sample groups.

Both the paired t-test and a Wilcoxon signedrank test were used to evaluate if there were statistically significant differences in the field-audit results based on the presence or absence of rinse water in the sample-collection bucket. The results of the paired t-test are presented in tables 11 and 12 and indicate that all analytes, with the exception of sodium and sulfate, yield the same results when comparing for statistically significant $(\alpha=0.05)$ differences between the "wet" and "dry" samples. The paired t-test showed that for "wet" coded samples there was a statistically significant difference for sodium (0.0363) but not for the "dry" coded samples (0.5694). Similarly, the analysis showed that there was a statistically significant difference for sulfate for the "dry" coded samples $(0.0009)$ but not for the "wet" coded samples $(0.0955)$. Results of the Wilcoxon signed-rank test indicated no statistically significant differences between the paired analyses for any of the analytes (tables 11 and 12) for either the "wet" or "dry" coded samples. Giving more weight to the Wilcoxon signed-rank test, which is slightly less powerful than the t-test for paired observations but more suitable for data sets that do not follow a normal distribution, combining "wet" and "dry" samples into one file was deemed appropriate.

Because there were 102 samples coded as "wet" and only 88 coded as "dry," a followup series of data-analysis experiments was completed to rule out the concern that differences in the sample populations were skewing the comparison of "wet" and "dry" samples. An equal number of "dry" and "wet" samples, with an equal distribution of the different matrix types in each group, were retained and analyzed using the Wilcoxon signed-rank test. In the normalization of the number of samples classified as "wet" or "dry," "wet" samples and "dry" samples were randomly selected and retained. The fact that five solutions and three volumes were used in the field-audit program during 1997 through 1999 was accounted for during the subsetting. The Wilcoxon signed-rank test was run on three data sets normalized in this manner, yielding virtually identical results each time, which is comparable to the Wilcoxon signed-rank test run on the entire field-audit sample population for 1997-99 without any subsetting. These results confirmed that the differences between the "wet" and "dry" samples were minor and did not preclude combining the "wet" and "dry" samples for the subsequent analyses in this report. A statistical summary of paired bucket minus bottle results for 1997-99 field-audit samples is shown in table 13. In addition to depicting the median paired bucket minus bottle differences for field-audit samples, table 13 also depicts the upper and lower quartiles. 


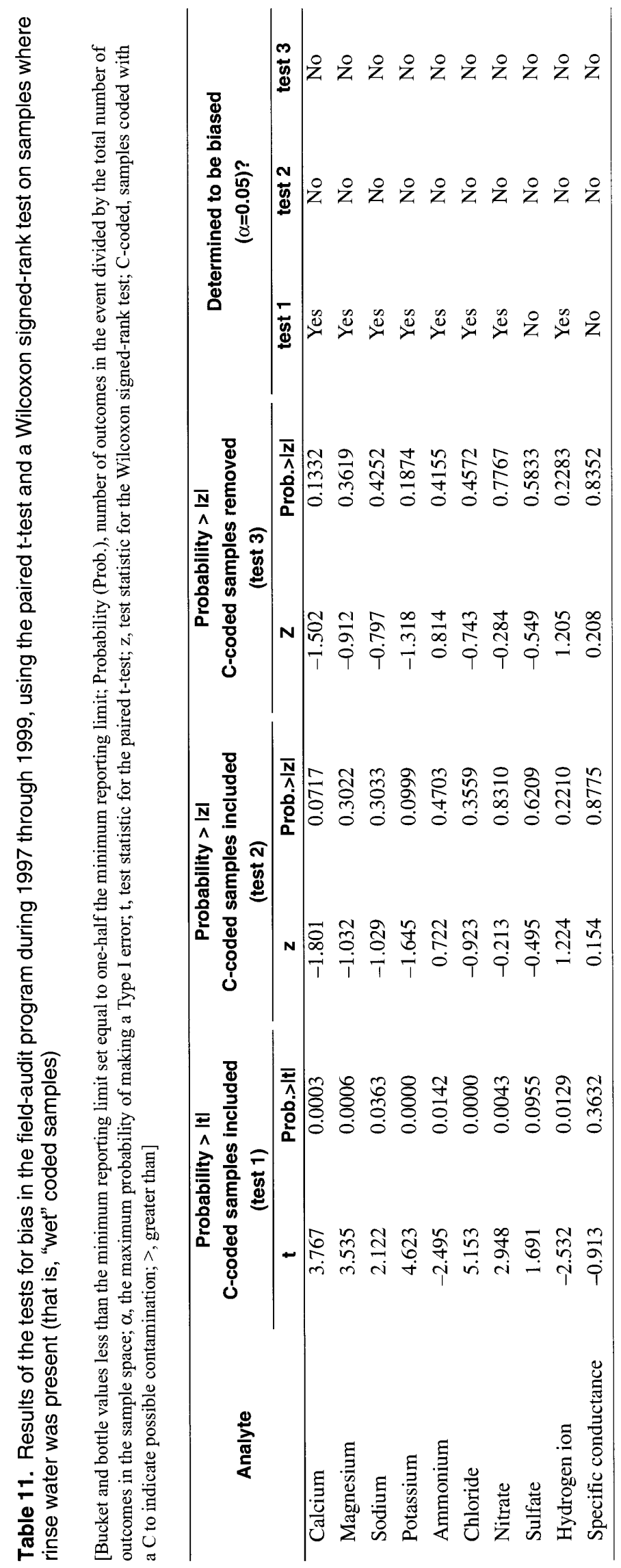




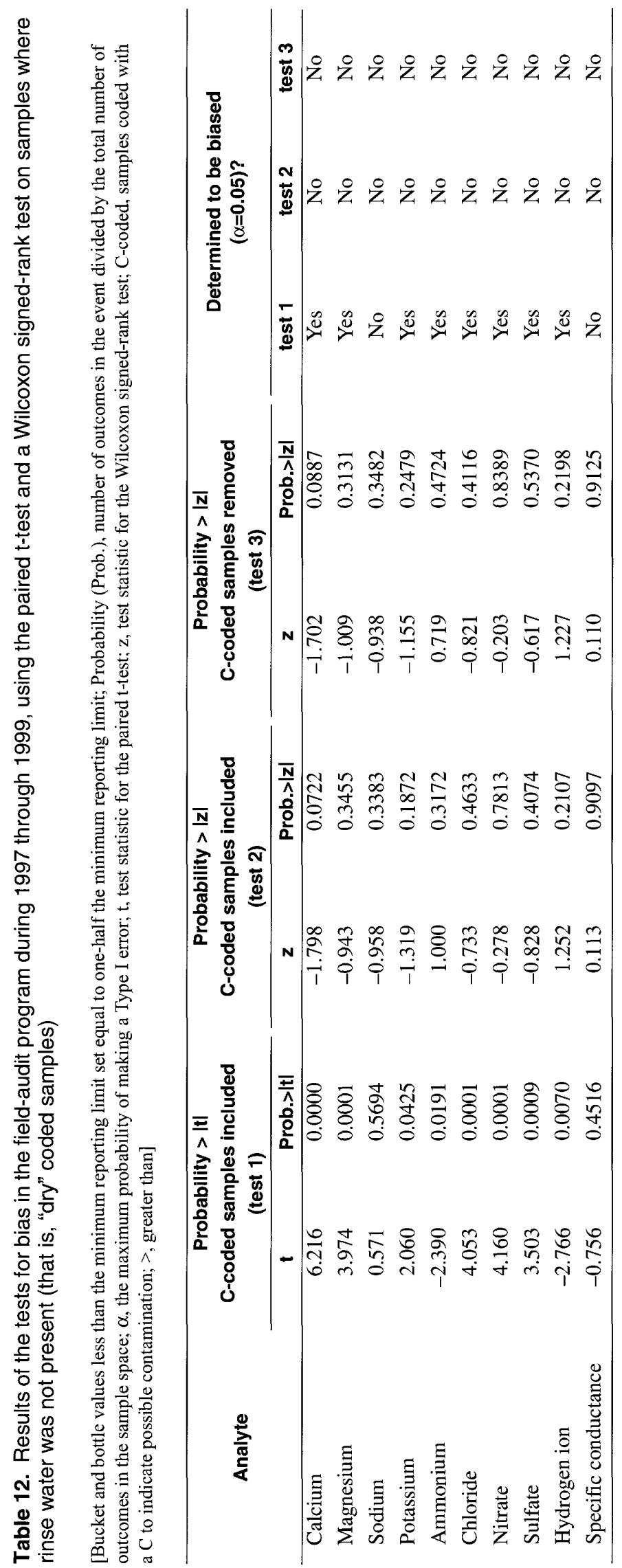


Table 13. Selected statistics for the paired bucket-sample concentration minus bottle-sample concentration differences in the field-audit program during 1997 through 1999 for "wet" and "dry" coded samples

[All units in milligrams per liter except hydrogen ion, in microequivalents per liter, and specific conductance, in microsiemens per centimeter at 25 degrees Celsius; N, number of samples; Q1, the lower quartile in the data distribution; Q3, the upper quartile in the data distribution]

\begin{tabular}{|c|c|c|c|c|c|c|}
\hline \multirow{3}{*}{ Analyte } & \multicolumn{3}{|c|}{ "Wet" samples } & \multicolumn{3}{|c|}{ "Dry" samples } \\
\hline & \multirow{2}{*}{ Median } & \multicolumn{2}{|c|}{ Quartiles } & \multirow{2}{*}{ Median } & \multicolumn{2}{|c|}{ Quartiles } \\
\hline & & Q1 & Q3 & & Q1 & Q3 \\
\hline Calcium & 0.006 & 0.000 & 0.016 & 0.010 & 0.000 & 0.024 \\
\hline Magnesium & 0.001 & 0.000 & 0.003 & 0.000 & 0.000 & 0.002 \\
\hline Sodium & 0.002 & -0.001 & 0.005 & 0.002 & 0.000 & 0.005 \\
\hline Potassium & 0.001 & 0.000 & 0.004 & 0.001 & 0.000 & 0.005 \\
\hline Ammonium & 0.000 & -0.010 & 0.000 & 0.000 & -0.020 & 0.000 \\
\hline Chloride & 0.010 & 0.000 & 0.020 & 0.010 & 0.000 & 0.020 \\
\hline Nitrate & 0.000 & 0.000 & 0.030 & 0.010 & 0.000 & 0.025 \\
\hline Sulfate & 0.000 & 0.000 & 0.020 & 0.006 & 0.000 & 0.020 \\
\hline Hydrogen ion & -0.687 & -2.063 & 0.000 & -0.906 & -1.881 & 0.000 \\
\hline Specific conductance & -0.200 & -0.600 & 0.100 & -0.100 & -0.400 & 0.200 \\
\hline
\end{tabular}

Boxplots in figure 18 graphically depict the paired bucket minus bottle concentration differences for all the major ions, as well as hydrogen ion and specific conductance for the field-audit program. The upper and lower lines defining the "box" portions of the boxplots depict the interquartile range of the differences for each analyte. The "whisker" portions of the boxplots are defined by the largest value within \pm 1.5 times the interquartile range extending from either the top or bottom of the "box" portion. The quartiles of the hydrogen-ion differences indicated that 50 percent of the samples experienced a change in hydrogen-ion concentration of between -2.063 and $0.000 \mu \mathrm{eq} / \mathrm{L}$ for "wet" coded samples and between -1.881 and $0.000 \mu \mathrm{eq} / \mathrm{L}$ for "dry" coded samples (table 13), a small percentage of the hydrogen-ion concentration present in the reference samples used in the program.

Figure 19 depicts paired field-audit differences by sample concentration. The paired field-audit differences for hydrogen ion and specific conductance, arranged by sample concentration, are shown in figure 20. Results of a Kruskal-Wallis analysis of variance test indicate a statistically significant relation between the magnitude of paired field-audit differences and concentration for magnesium, chloride, and nitrate in 1998 and in 1999 (table 14). Although there were no statistically significant relations between the magnitude of paired field-audit differences and concentration in 1997, it is important to remember that a much smaller number of samples were analyzed in
1997, reflecting the July 1997 startup date for the field-audit program. In 1998, a significant relation between the magnitude of paired field-audit differences and concentrations was found for magnesium, chloride, nitrate, and sulfate. In 1999, calcium, magnesium, chloride, nitrate, and specific conductance showed a statistically signficant relation between the magnitude of paired field-audit differences and the concentrations of these analytes.

Relative and absolute percent differences were calculated for all of the paired field-audit samples. The upper and lower quartiles as well as the median relative and absolute percent differences during 1997 through 1999 are listed in table 15 . The relative and absolute percent differences were determined by calculating each paired field-audit difference as a percentage of the known sample concentration using the same process used in the blind-audit program (equations 3 and 4).

In 1997-99, the median (50th percentile) relative percent differences for all analytes was within a range of -5.36 (hydrogen ion) to +5.56 (chloride) percent, indicating minor positive or negative bias (table 15). The median (50th percentile) absolute percent differences for the field-audit program, similar to the blind-audit program, did not exceed 9 percent for any of the analytes. The analyte with the largest median absolute percent difference in the field-audit program was potassium (8.70). Potassium also had the largest median absolute percent difference (7.14) in the blind-audit program (table 8 ). 


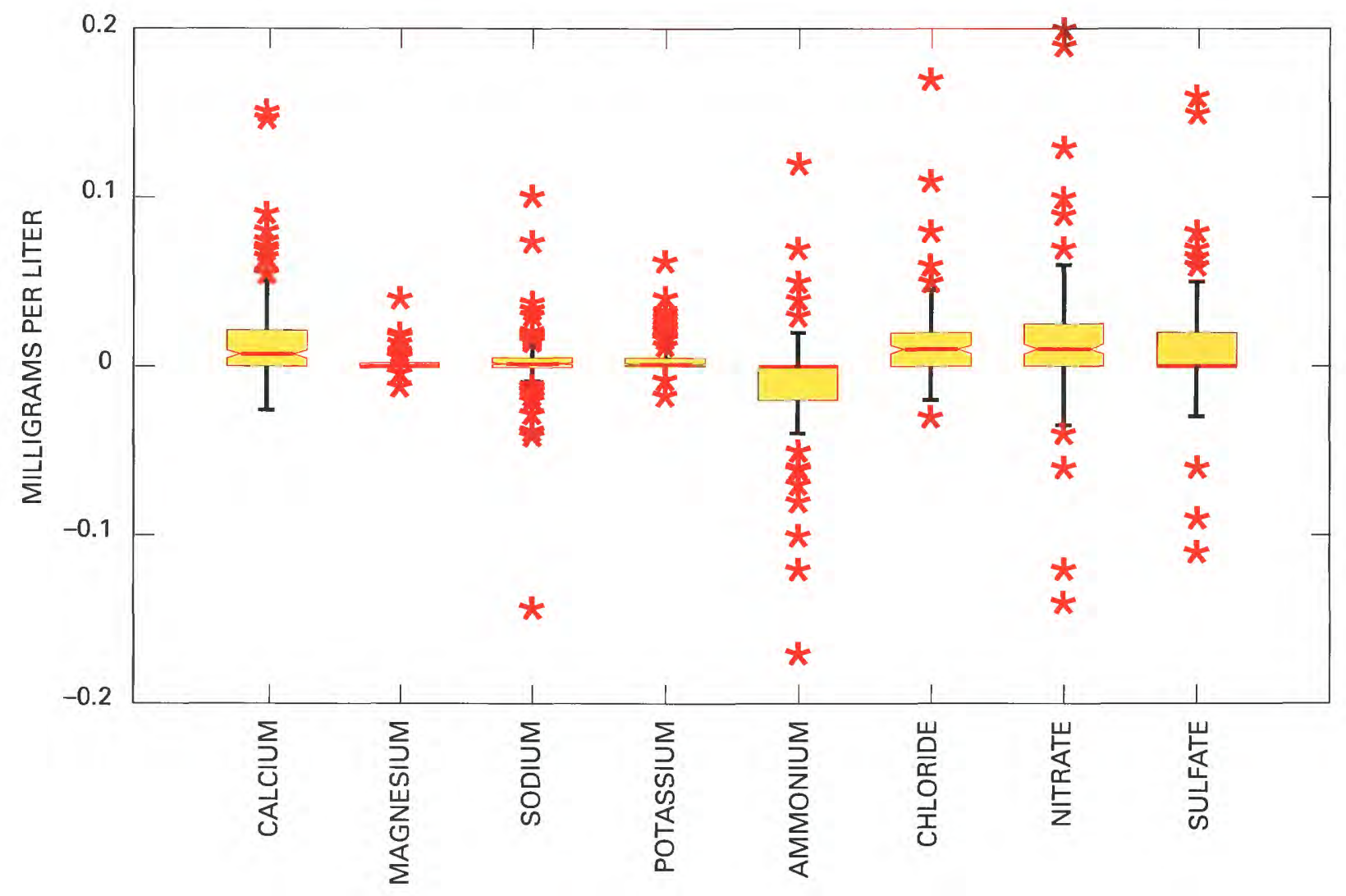

\section{EXPLANATION}
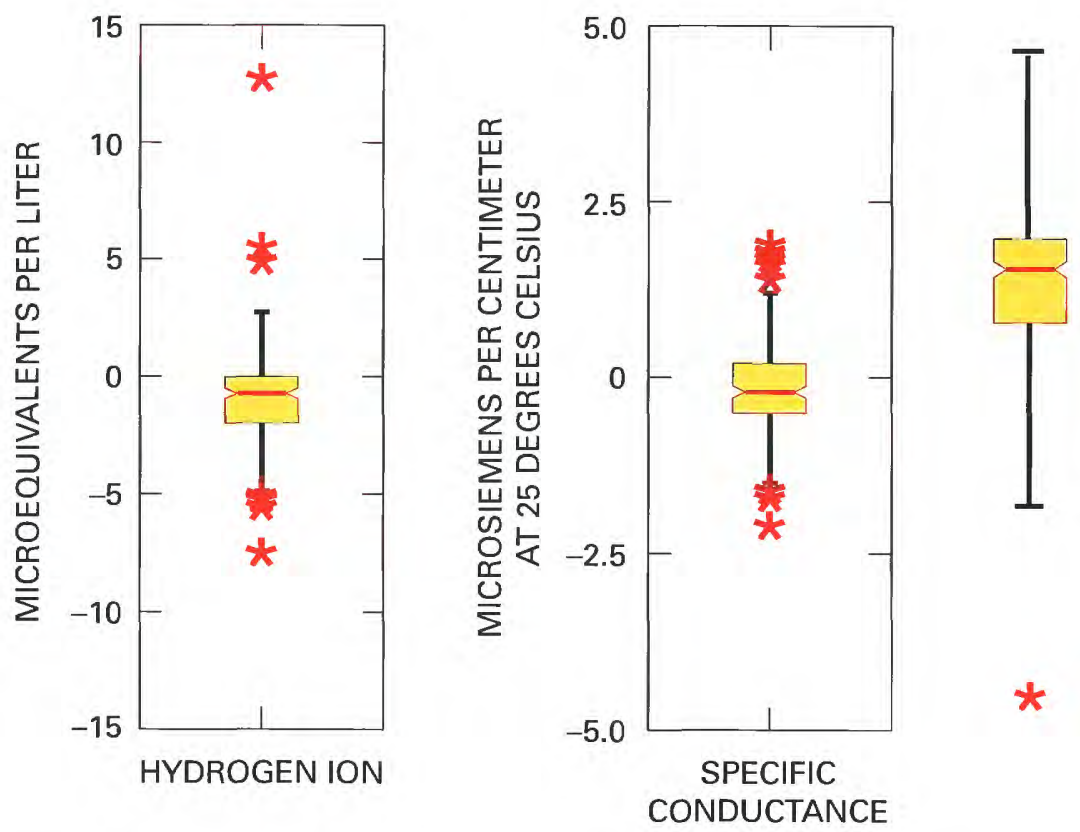

Largest value less than or equal to the 75 th percentile plus 1.5 times the interquartile range

upper quartile

Median

Lower quartile

Smallest value less than or equal to the 25th percentile minus 1.5 times the interquartile range

Remaining 1 percent of the data not inclusive in the distribution tails

Figure 18. Paired bucket-sample concentrations minus bottle-sample concentrations in the field-audit program during 1997 through 1999. 

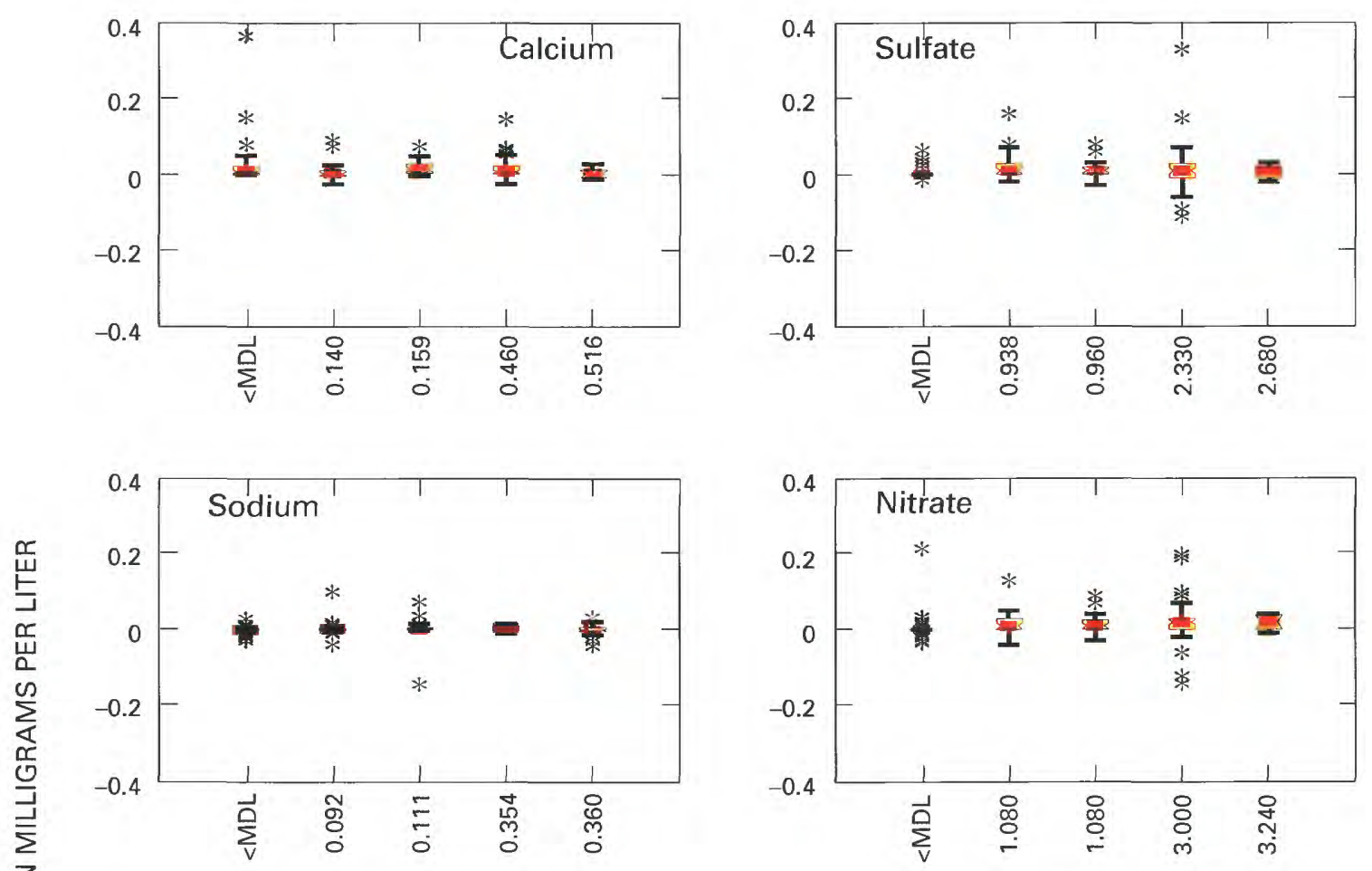

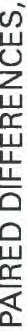
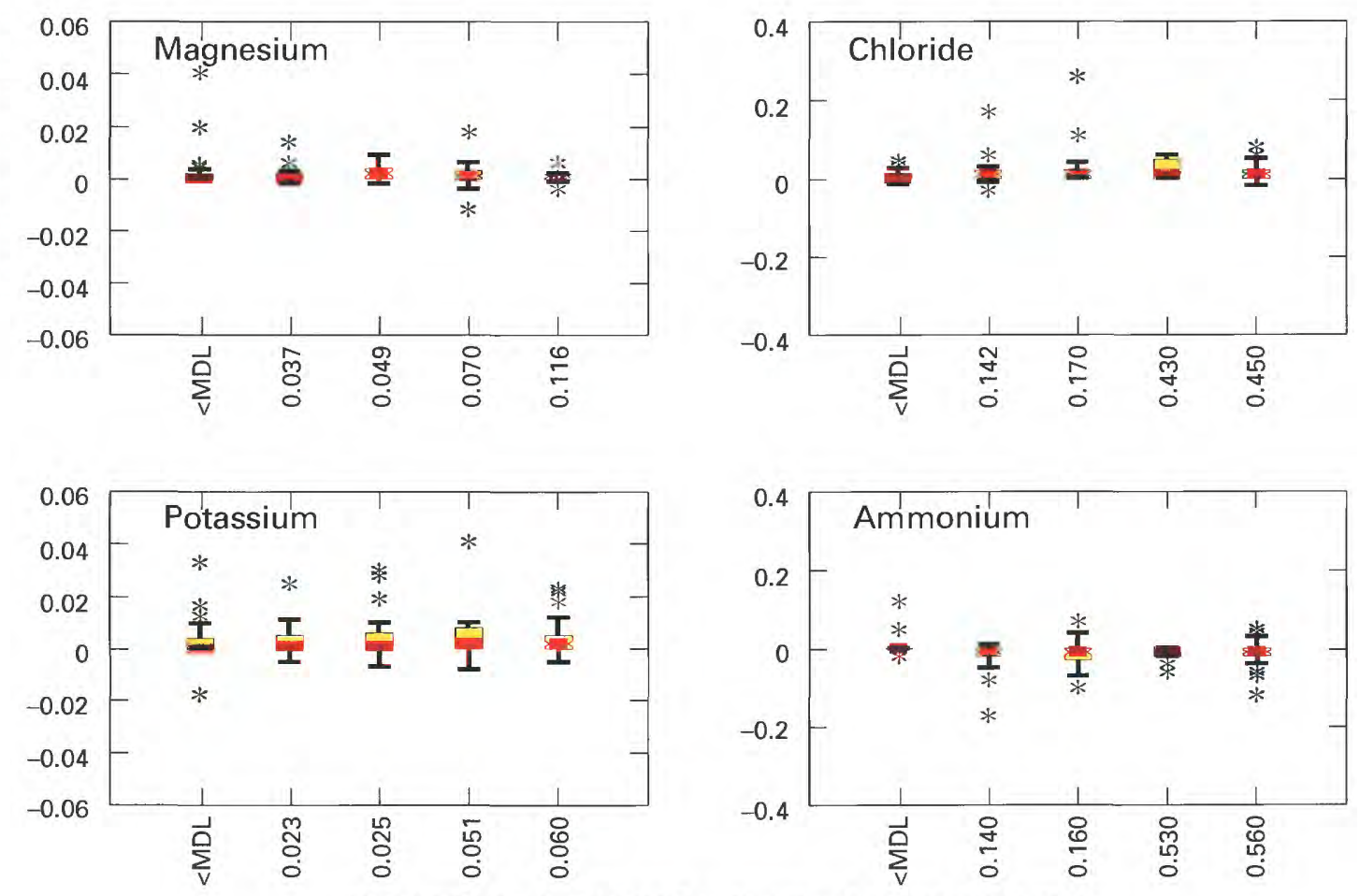

TARGET CONCENTRATION, IN MILLIGRAMS PER LITER

\section{EXPLANATION}

$<$ MDL Target less than method detection limit

* $\quad$ Remaining 1 percent of data not inclusive in the distribution tails

Figure 19. Relation between paired field-audit differences for major ions and the analyte concentrations of solutions used in the field-audit program during 1997 through 1999. 

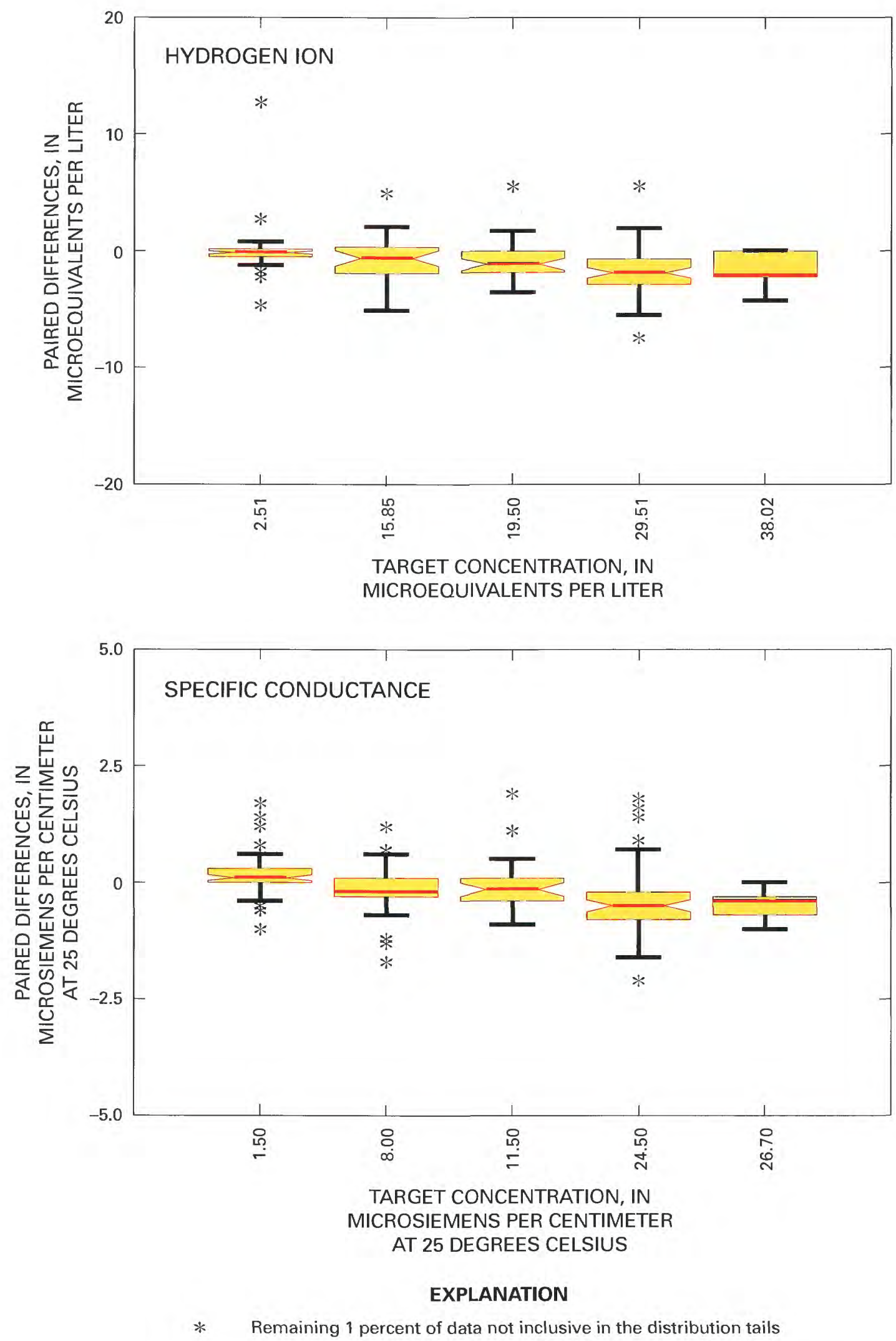

Figure 20. Relation between paired field-audit differences for hydrogen ion and specific conductance and the analyte concentrations of solutions used in the field-audit program during 1997 through 1999. 
Table 14. Results of the Kruskal-Wallis analysis of variance test to determine the relation between paired field-audit sample differences and the target concentrations used in the field-audit program in 1997, 1998, and 1999

[All paired field-audit differences expressed in milligrams per liter, except specific conductance in microsiemens per centimeter at 25 degrees Celsius and hydrogen ion in microequivalents per liter]

\begin{tabular}{|c|c|c|c|c|c|c|}
\hline \multirow[t]{2}{*}{ Analyte } & \multicolumn{3}{|c|}{$\begin{array}{l}\text { Bucket minus bottle differences } \\
\text { in significance levels ( } p \text {-values) } \\
\text { by target concentration }\end{array}$} & \multicolumn{3}{|c|}{$\begin{array}{l}\text { Statistically significant } \\
\qquad(\alpha=0.05) ?\end{array}$} \\
\hline & 1997 & 1998 & 1999 & 1997 & 1998 & 1999 \\
\hline Calcium & 0.1674 & 0.2809 & 0.0135 & No & No & Yes \\
\hline Magnesium & 0.5870 & 0.0137 & 0.0136 & No & Yes & Yes \\
\hline Sodium & 0.3610 & 0.2557 & 0.8586 & No & No & No \\
\hline Potassium & 0.7312 & 0.4159 & 0.6234 & No & No & No \\
\hline Ammonium & 0.8062 & 0.0706 & 0.0840 & No & No & No \\
\hline Chloride & 0.6542 & 0.0002 & 0.0198 & No & Yes & Yes \\
\hline Nitrate & 0.5150 & 0.0433 & 0.0160 & No & Yes & Yes \\
\hline Sulfate & 0.1910 & 0.0092 & 0.1743 & No & Yes & No \\
\hline Hydrogen ion & 0.1096 & 0.6021 & 0.2359 & No & No & No \\
\hline Specific conductance & 0.0598 & 0.1068 & 0.0008 & No & No & Yes \\
\hline
\end{tabular}

Table 15. Relative and absolute bucket minus bottle differences calculated as a percentage of the target concentration for the bottle portion for each analyte during 1997 through 1999 for the field-audit program

\begin{tabular}{|c|c|c|c|c|c|c|}
\hline \multirow{3}{*}{ Analyte } & \multicolumn{3}{|c|}{$\begin{array}{l}\text { Relative bucket minus bottle differences, } \\
\text { expressed as a percentage of corresponding target } \\
\text { bottle concentration (selected data pairs only) }\end{array}$} & \multicolumn{3}{|c|}{$\begin{array}{l}\text { Absolute bucket minus bottle differences, } \\
\text { expressed as a percentage of corresponding target } \\
\text { bottle concentration (selected data pairs only) }\end{array}$} \\
\hline & \multicolumn{3}{|c|}{ Percentiles } & \multicolumn{3}{|c|}{ Percentiles } \\
\hline & 25th & 50th & 75th & 25th & 50th & 75th \\
\hline Calcium & 0.00 & 3.57 & 12.50 & 1.24 & 4.88 & 12.80 \\
\hline Magnesium & 0.00 & 0.00 & 4.29 & 0.00 & 2.13 & 5.71 \\
\hline Sodium & -0.42 & 1.22 & 4.12 & 0.81 & 2.85 & 7.32 \\
\hline Potassium & 0.00 & 3.33 & 18.48 & 0.00 & 8.70 & 21.74 \\
\hline Ammonium & -7.14 & 0.00 & 0.00 & 0.00 & 6.67 & 13.33 \\
\hline Chloride & 0.00 & 5.56 & 11.11 & 0.00 & 5.56 & 11.11 \\
\hline Nitrate & 0.00 & 0.33 & 1.89 & 0.00 & 0.94 & 2.18 \\
\hline Sulfate & 0.00 & 0.00 & 2.08 & 0.00 & 1.05 & 2.11 \\
\hline Hydrogen ion & -11.60 & -5.36 & 0.00 & 2.26 & 6.91 & 12.46 \\
\hline Specific conductance & -3.84 & -1.10 & 1.79 & 1.74 & 3.09 & 6.25 \\
\hline
\end{tabular}


Table 16. Results of the 1997 through 1999 Kruskal-Wallis analysis of variance tests to determine if bucket minus bottle differences for the 250-, 500-, and 1,000-milliliter samples of the USGS solution used in the field-audit program have equivalent distributions

[mL, milliliter]

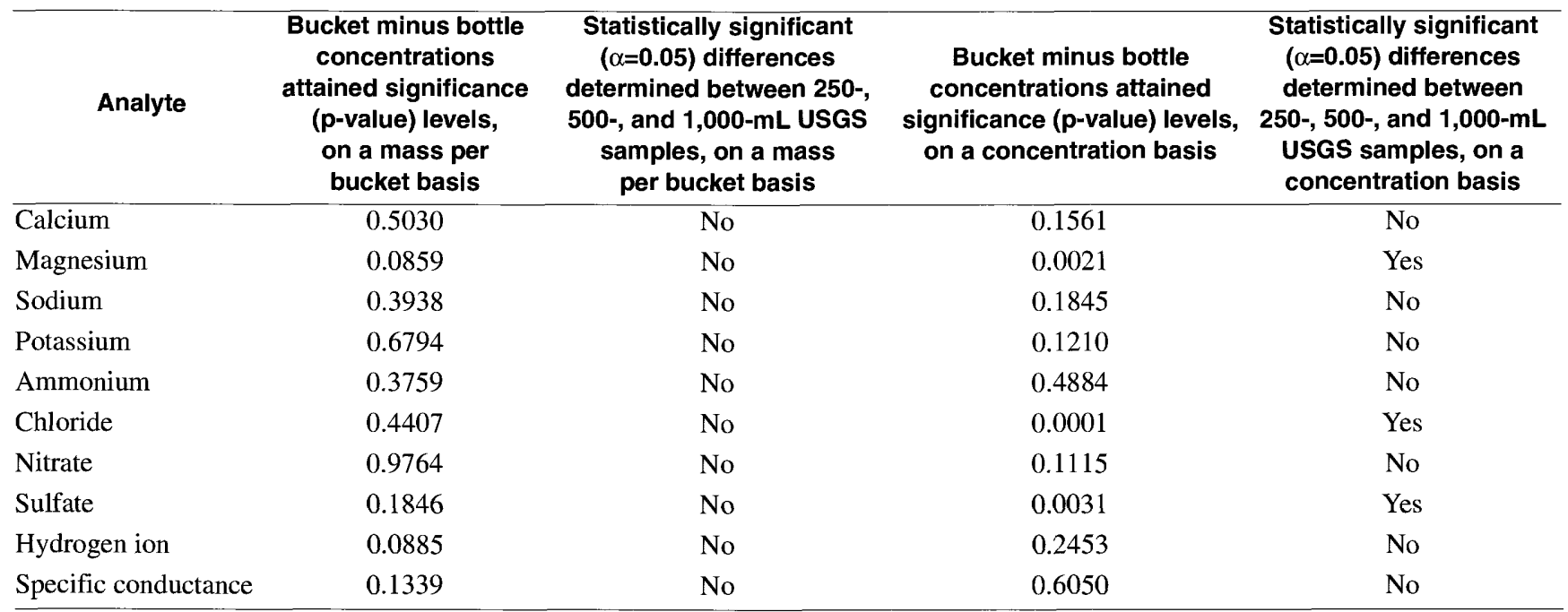

With the intent of replicating the wide range of sample volume and sample chemistry at the NADP/NTN sites, a 3 by 3 sample design (Berthouex and Brown, 1995) was chosen. Three different sample volumes and three different sample matrixes were used in the field-audit program to investigate a possible relation between sample volume collected weekly at NADP/NTN sites and the amount of contamination introduced through shipping and handling procedures. An equal number of samples with 250,500 , or $1,000 \mathrm{~mL}$, using the three different matrixes including Ultrapure deionized water, were distributed to 25 sites per quarter. Differences between bucket and bottle pairs were analyzed in their original concentration units and in units of mass. Boxplots in figures 21 and 22 depict the differences for each analyte, plotted by sample volume in units of mass.

To determine if there was a statistically significant relation between paired field-audit differences and sample volume, a Kruskal-Wallis analysis of variance test was performed. An equal number of samples containing 250,500 , or $1,000 \mathrm{~mL}$ of solution were included in each quarterly mailing throughout 1997-99. Kruskal-Wallis results for 1997-99 indicate a significant $(\alpha=0.05)$ relation between paired fieldaudit sample differences in units of concentration and sample volume for magnesium, chloride, and sulfate (table 16). Results for these same analytes also indicated a significant difference in the blindaudit program (table 9). When paired differences were converted to units of mass, the outcome of the Kruskal-Wallis test was markedly different: no statistically significant $(\alpha=0.05)$ differences were found for any of the analytes during 1997-99.

\section{Ultrapure Deionized-Water Sample Results}

The number of Ultrapure deionized-water samples analyzed as part of the field-audit program was 11 in 1997, 22 in 1998, and 24 in 1999. Ultrapure deionized-water field-audit samples were processed in two parts: the first part, the bucket portion, was exposed to normal sample-handling steps, representing the field-exposed portion, and the second part, the bottle portion, was subject to minimal handling, representing the control portion. The most commonly detected analyte during 1997 in the Ultrapure deionized-water samples was sodium, which was detected in all (11 of 11) samples in the field-exposed portion and in the control portion (table 17). Sodium detection in 1998 decreased and was found in 14 of the 22 samples in the field-exposed portion and in only 2 of 22 samples in the control portion. Sodium detection decreased again in 1999 with sodium found in only 12 of 24 samples in the field-exposed portion, and sodium was not detected at all in the minimally handled control portion of the samples. As discussed previously in the blind-audit section of this report, the high incidence of sodium 

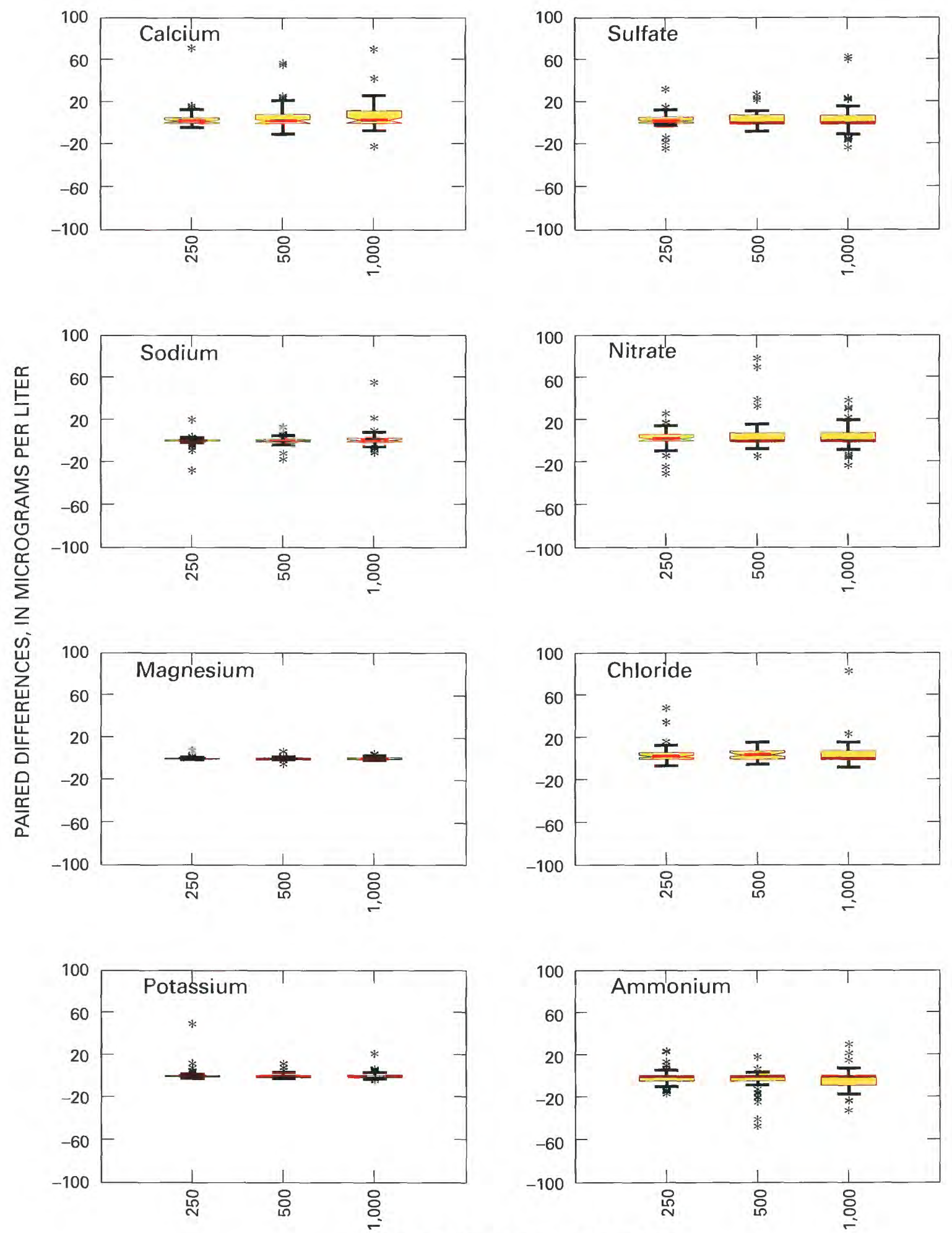

SAMPLE VOLUME, IN MILLILITERS

EXPLANATION

* Remaining 1 percent of data not inclusive in the distribution tails

Figure 21. Relation between paired field-audit differences for the major ions and sample volume for the 250-, 500-, and 1,000-milliliter U.S. Geological Survey solution samples during 1997 through 1999. 


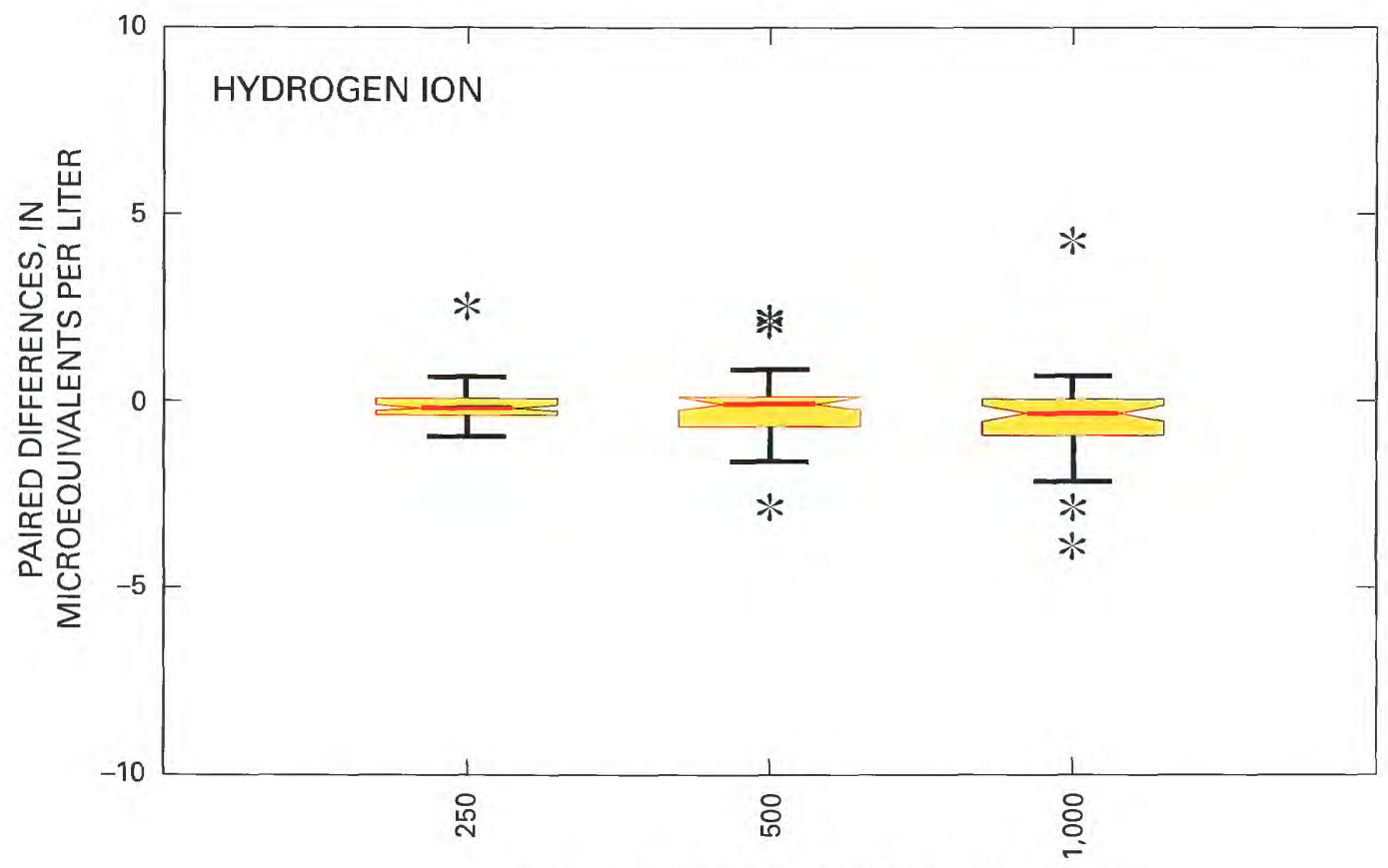

SAMPLE VOLUME, IN MILLILITERS

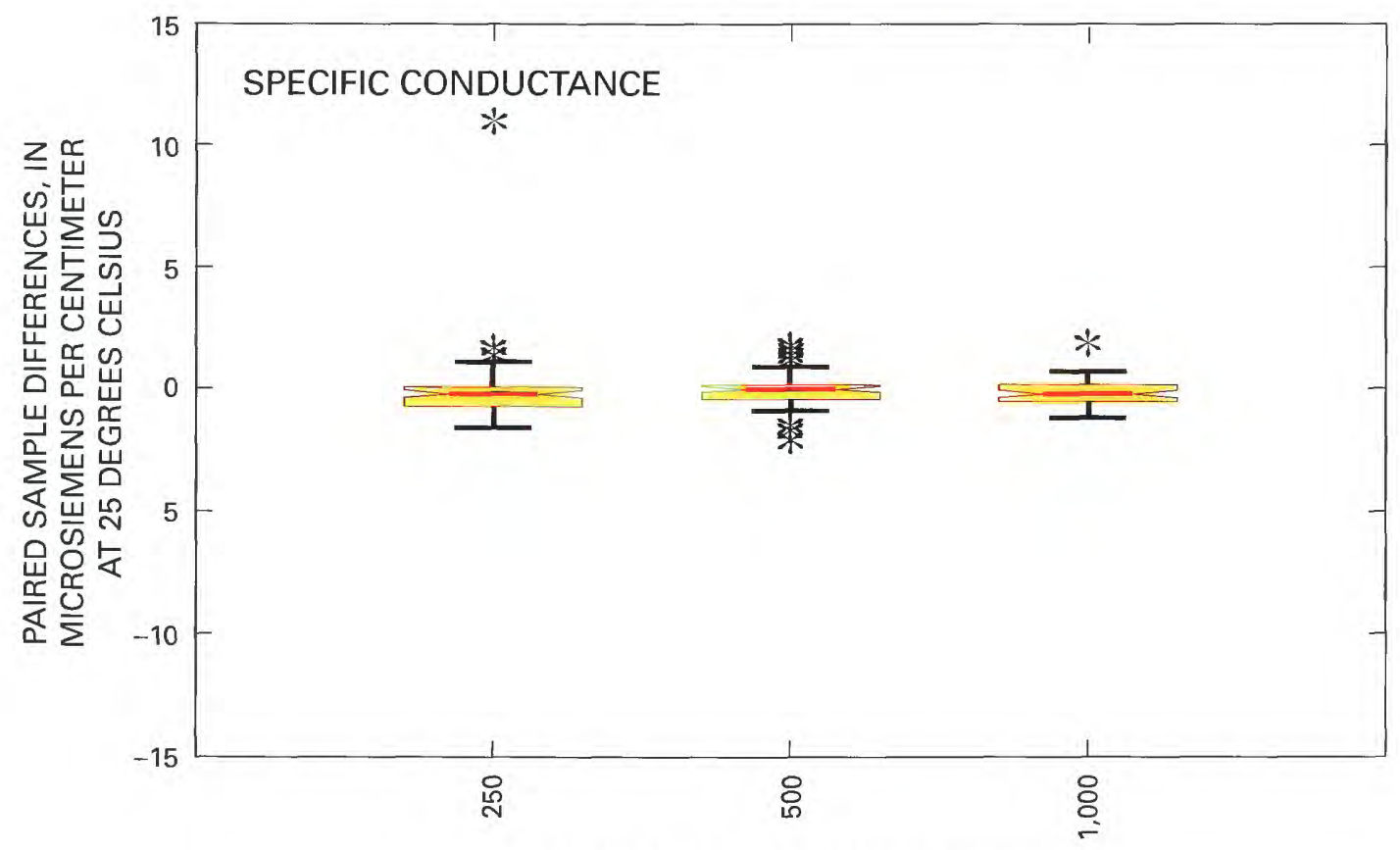

SAMPLE VOLUME, IN MILLILITERS

\section{EXPLANATION}

* Remaining 1 percent of data not inclusive in the distribution tails

Figure 22. Relation between paired field-audit differences for hydrogen ion and specific conductance and sample volume for 250-, 500-, and 1,000-milliliter U.S. Geological Survey solution samples during 1997 through 1999. 
Table 17. Number of determinations exceeding the minimum reporting limit for the 11 Ultrapure deionized-water samples submitted in 1997, the 22 samples submitted in 1998, and the 24 submitted in 1999 as part of the field-audit program

\begin{tabular}{lrrrrrrrr}
\hline \multirow{2}{*}{ Analyte } & \multicolumn{3}{c}{$\begin{array}{c}\text { Field-exposed } \\
\text { bucket portion }\end{array}$} & & \multicolumn{3}{c}{$\begin{array}{c}\text { Minimally handled } \\
\text { bottle portion }\end{array}$} \\
\cline { 2 - 3 } \cline { 7 - 8 } & $\mathbf{1 9 9 7}$ & $\mathbf{1 9 9 8}$ & $\mathbf{1 9 9 9}$ & & $\mathbf{1 9 9 7}$ & $\mathbf{1 9 9 8}$ & $\mathbf{1 9 9 9}$ \\
\hline Calcium & 6 & 10 & 20 & & 3 & 2 & 3 \\
Magnesium & 4 & 6 & 9 & & 1 & 0 & 0 \\
Sodium & 11 & 14 & 12 & & 11 & 2 & 0 \\
Potassium & 3 & 8 & 8 & & 0 & 2 & 0 \\
Ammonium & 0 & 1 & 2 & & 1 & 0 & 0 \\
Chloride & 8 & 4 & 6 & & 5 & 0 & 0 \\
Nitrate & 9 & 5 & 3 & & 10 & 0 & 0 \\
Sulfate & 2 & 2 & 5 & & 0 & 0 & 1 \\
\hline
\end{tabular}

detection in the Ultrapure samples during 1997 is due to the type of filters used at the CAL to process the samples until November 1997. After sodium, calcium was the next most commonly detected analyte in the field-exposed portion of the field-audit program between 1997 and 1999: in the field-exposed sample portions, calcium was detected in 6 of 11 samples in 1997, in 10 of 22 samples in 1998, and in 20 of 24 samples in 1999. The incidence of nitrate detections in the field-exposed portions of the Ultrapure samples decreased steadily over the 3 years analyzed for this report, from 9 of 11 samples in 1997 to 5 of 22 samples in 1998 and to 3 of 24 samples in 1999. Sodium and nitrate were both commonly detected in the minimally handled bottle portion in 1997 ( 11 of 11 for sodium and 10 of 11 for nitrate). None of the analytes had a detection rate greater than 2 of 22 samples for the minimally handled bottle portion portion in 1998. The only ions detected in 1999 in the minimally handled bottle portion of the field-audit samples were calcium ( 3 of 24) and sulfate (1 of 24).

\section{INTERLABORATORY-COMPARISON PROGRAM}

The two objectives of the interlaboratorycomparison program are the following: (1) to determine if statistically significant differences exist (bias) among the analytical results of participating laboratories, and (2) to estimate the analytical precision of participating laboratories. The following laboratories participated in the interlaboratory-comparison program during 1997 through 1999: (1) Illinois State Water Survey, Central
Analytical Laboratory (CAL) in Champaign, Ill.; (2) Meteorological Services of Canada (MSC) in Ontario, Canada; (3) Environmental Science and Engineering, Inc. (ESE) in Gainesville, Fla.; (4) Ontario Ministry of the Environment, Dorset Research Facility (MOE) in Ontario, Canada; and (5) Shepard Analytical Services (SA) in Simi Valley, Calif. ESE did not participate in the program during January 26, 1999, through June 2, 1999. On June 21, 1999, the USGS began measuring analytical data quality from wet-deposition chemistry laboratories in Europe and Southeast Asia when the following laboratories joined the program: (6) Norwegian Institute for Air Research (NILU) in Kjeller, Norway, and (7) Acid Deposition and Oxidant Research Center (ADORC) in Niigata-shi, Japan. The addition of the NILU and ADORC laboratories was beneficial because it meant that for the first time, all of the major global atmospheric-deposition monitoring networks were united in a single program designed to measure laboratory data quality. These laboratories joined those representing the major North American deposition-monitoring networks already in the program, making it possible to compare data directly from wellknown deposition-monitoring networks around the world. In addition to monitoring trends in acid rain, nitrogen deposition data from these networks are being combined as input to global circulation models to estimate the effects of nitrogen deposition on the global cycling of carbon dioxide.

Four sources of samples were used in the interlaboratory-comparison program during 1997 through 1999: (1) synthetic wet-deposition samples and Ultrapure deionized-water samples prepared by the USGS; (2) a standard reference sample prepared and certified by the U.S. National Institute of Standards and Technology (NIST) (National Institute of Standards and Technology, 1991); (3) NIST-traceable standard reference samples prepared by High Purity Standards (HPS); and (4) natural wet-deposition samples collected at NADP/NTN sites and bottled by the CAL. Table 3 contains information on the preparation of the solutions made either by the USGS or the CAL, as well as the solution names of the NIST-certified and NISTtraceable samples. Target values for all of the synthetic wet-deposition solutions used in the interlaboratorycomparison program are listed in table 4.

In 1997 , each participating laboratory was sent 104 samples. Of the 104 samples, 52 were aliquots of natural precipitation bottled by the CAL, 12 were synthetic samples made by the USGS and referred to 
as "USGS solution," 6 were NIST-certified samples (2694-A-II), 8 were synthetic samples made by the USGS and referred to as "SP-1 solution," 8 were synthetic samples made by the USGS and referred to as "SP-2 solution," 6 were NIST-traceable samples made by HPS and referred to as "SP-97 solution," 6 were NIST-traceable samples made by HPS and referred to as "SP-98 solution," and 6 were Ultrapure deionized-water samples bottled by the USGS.

In 1998, each laboratory was once again sent 104 samples to analyze. Of the 104 samples, 52 were aliquots of natural precipitation bottled by the CAL, 12 were synthetic samples made by the USGS and referred to as "SP-3 solution," 9 were NIST-traceable samples made by HPS and referred to as "SP-97 solution," 9 were NIST-traceable samples made by HPS and referred to as "SP-98 solution," 8 were synthetic samples made by the USGS and referred to as "SP-4 solution," 6 were synthetic samples made by the USGS and referred to as "SP-5 solution," and 8 were Ultrapure deionizedwater samples bottled by the USGS.

To be consistent with previous years, each laboratory was once again sent 104 samples to analyze in 1999. Of the 104 samples, 52 were aliquots of natural precipitation bottled by the CAL, 10 were NISTtraceable samples made by HPS and referred to as "SP-97 solution," 10 were NIST-traceable samples made by HPS and referred to as "SP-98 solution," 8 were NIST-traceable samples made by HPS and referred to as "SP-5 solution," 8 were synthetic samples made by the USGS and referred to as "SP-1 solution," 8 were synthetic samples made by the USGS and referred to as "SP-2 solution," and 8 were Ultrapure deionized-water samples bottled by the USGS. The solutions made by the USGS for 1998 were assigned different names from those used in 1997 because the measured concentrations differed from the expected concentrations for one or more analytes. Samples used in the interlaboratory-comparison program were relabeled and shipped by the USGS to the participating laboratories biweekly. Each laboratory received four samples per shipment.

Natural wet-deposition samples collected at NADP/NTN sites with sufficient excess volume (samples in excess of $750 \mathrm{~mL}$ ) were selected randomly by the CAL for use in the interlaboratory-comparison program and divided into 10 aliquots using a decasplitter (Gordon, 1999). Aliquots from the split sample were bottled in $125-\mathrm{mL}$ polyethylene bottles and shipped in chilled, insulated containers to the USGS in Denver, Colo. The USGS kept these natural samples refrigerated and shipped the samples on ice to participating laboratories within a few weeks of receiving them. With the exception of the natural samples, chemical concentrations of the solutions were known either because the sample was a certified reference sample or because it was prepared by the USGS and tested to verify the target values. Analysis of the natural samples was limited to comparing differences among laboratories on identical sets of replicate samples (26 replicate pairs per laboratory) in a given year. A flowchart of the interlaboratory-comparison program is shown in figure 23 .

\section{Interlaboratory Bias}

Interlaboratory bias (bias among multiple laboratories) is defined as a systematic difference in reported values for a given laboratory observed when the results from several laboratories are compared. To examine potential bias in the analytical results among the laboratories, a Friedman test (SAS Institute Inc., 1989) was performed. A Friedman test investigates the significance of the differences in response to multiple treatments for more than two subjects without assuming the data are from normal distributions (Kanji, 1993). In this application, the multiple treatments are the different sample matrixes and the subjects are the various laboratories. This allows the comparison of paired data from each of the participating laboratories while controlling for the different sample matrixes sent in different mailings.

In 1997, results of the Friedman test indicated significant $(\alpha=0.05)$ differences in analyte measurements among the five laboratories for all analytes with the exception of potassium. However, the multiplecomparison test showed differences in the results for all analytes including potassium on an overall basis from all laboratories. Looking more closely at the results from the multiple-comparison test, the following differences between laboratory results for the various analytes were noted: no significant difference $(\alpha=0.05)$ between MOE and MSC for calcium, magnesium, sodium, and chloride; no significant differences between MSC and SA for magnesium, sodium, 


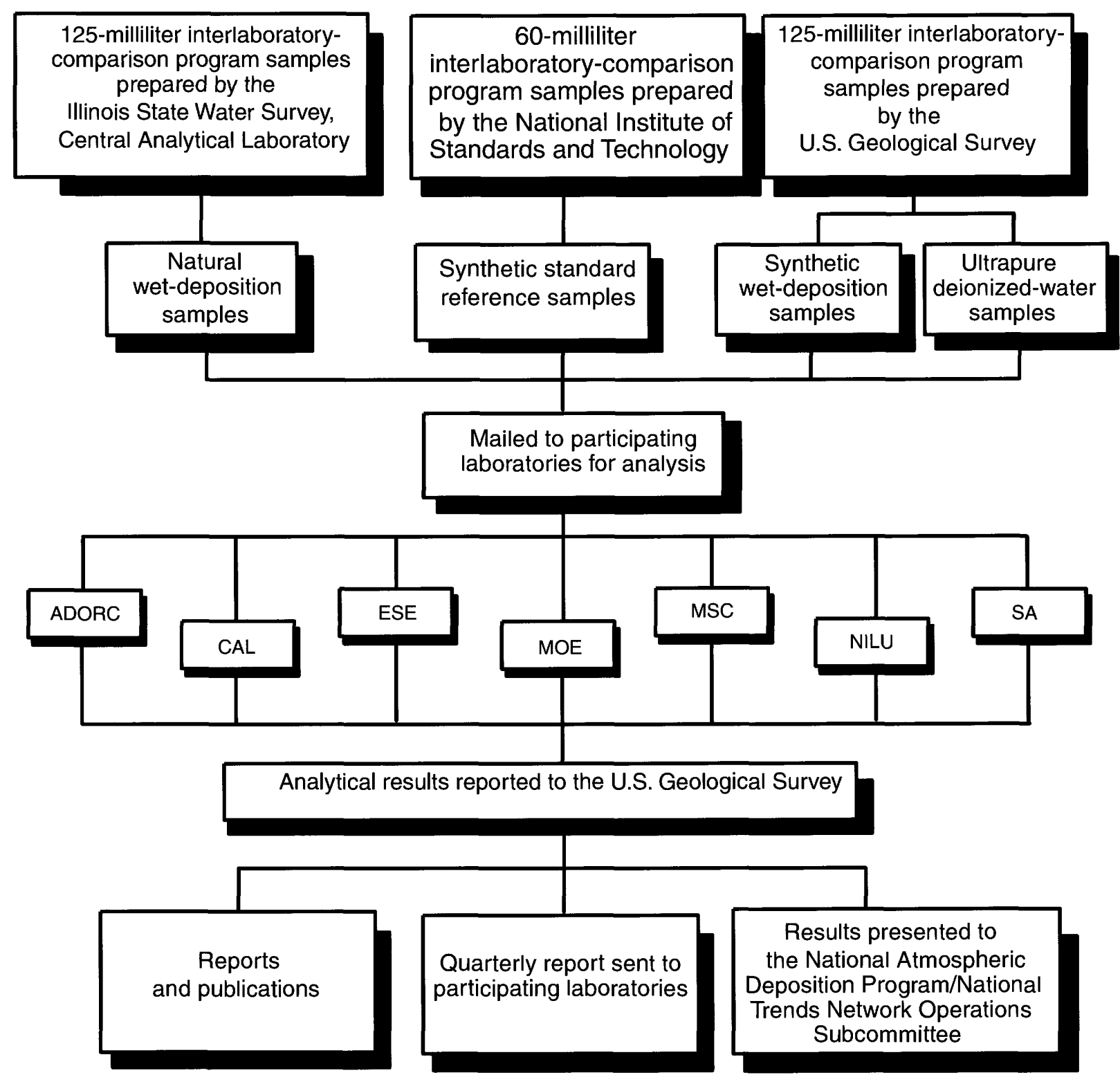

$\begin{array}{ll}\text { ADORC: } & \text { Acid Deposition and Oxidant Research Center, Niigata-shi, Japan } \\ \text { CAL: } & \text { Illinois State Water Survey, Central Analytical Laboratory, Champaign, Illinois } \\ \text { ESE: } & \text { Environmental Science and Engineering, Inc., Gainesville, Florida } \\ \text { MOE: } & \text { Ontario Ministry of the Environment, Dorset Research Facility (MOE), Ontario, Canada } \\ \text { MSC: } & \text { Meteorological Services of Canada, Ontario, Canada } \\ \text { NILU: } & \text { Norwegian Institute for Air Research, Kjeller, Norway } \\ \text { SA: } & \text { Shepard Analytical Services, Simi Valley, California }\end{array}$

Figure 23. Interlaboratory-comparison program of the U.S. Geological Survey.

ammonium, and sulfate. Also, no significant differences were found for any of the laboratories compared to any other laboratory (except CAL) for potassium. Comparisons between ESE and MOE and MSC, and between MOE and SA did not show a significant difference for chloride. No significant difference was found between SA and MOE for ammonium.
In 1998, the Friedman test indicated significant $(\alpha=0.05)$ differences in analyte measurements for all of the analytes. The multiple-comparison test further showed that, for sodium and chloride, a significant difference $(\alpha=0.05)$ was found for all laboratories compared to all other laboratories except between MOE and MSC and between MSC and SA. There 
was no significant difference between MOE and ESE for magnesium, potassium, and sulfate. ESE and SA showed no significant difference for magnesium, potassium, and ammonium. No significant difference was found between SA and MOE for potassium and ammonium. Comparisons between ESE and MSC and between MSC and SA showed no significant difference for ammonium.

The Friedman test indicated there were statistically significant $(\alpha=0.05)$ differences in the results from the seven laboratories for all analytes in 1999. Again, the multiple-comparison test was used to take a closer look at these differences. The test showed that there was no significant difference between MSC and SA for sodium, potassium, ammonium, chloride, nitrate, and sulfate. MSC compared to NILU showed no significant difference for the same analytes except for ammonium but did show differences for calcium and magnesium. No significant difference was found between SA and NILU or between SA and ADORC for calcium, magnesium, chloride, and nitrate. SA compared to NILU indicated no significant difference for sodium and ammonium, and SA compared to ADORC also indicated no significant difference for potassium and sulfate. MSC compared to ADORC showed no significant difference for ammonium, chloride, and sulfate. ADORC compared to NILU showed no significant difference for chloride.

In order to facilitate a visual comparison of interlaboratory differences, graphs of each laboratory's analyte concentrations minus the analyte medians calculated for all laboratories are presented in the control charts shown in figures 24-30. The control limits were placed at \pm 3 F-pseudosigmas from the zero difference line. Control limits (3-sigma) define the bounds of virtually all values ( 99 percent) produced by a system in statistical control. Modern control charts commonly have additional limits called warning limits (2-sigma) within which most (95 percent) of the values should lie (Taylor, 1987). The warning limits are positioned at \pm 2 F-pseudosigmas from the zero difference line.

There are some important features in figures 24-30 of which the reader should be aware. First, the graph for each analyte is scaled to reflect the maximum scatter for a given analyte in the data for one of the seven participating laboratories. The graph for each analyte is therefore scaled to reflect the maximum range of difference between a participating laboratory's measured values and the median value of all participating laboratories. When viewing figures 24-30, the different scale for each analyte should be noted. Scaling differences serve as visual clues of performance differences in the analysis of a given analyte for the laboratories as a group. For example, the graphs for sulfate and nitrate are scaled the widest of any of the graphs for the major ions, indicating that as a group the laboratories had the most variability in measured minus median values for these two analytes. It is also important to note that the scales for a given analyte are consistent in figures 24-30. This facilitates comparison of performance in the analysis of a given analyte among laboratories. This approach has its drawbacks in that comparison of a given laboratory's performance measuring various analytes puts an extra burden on the reader to adjust to changing analyte scales. However, if all the graphs had been scaled to facilitate comparison of analytes, comparison of laboratory performance would be difficult, as the scaling of the graphs would obscure these differences.

Graphs in figure 31 depict the number of data points outside the control limits for the CAL in 1997, 1998, and 1999. Graphs for other laboratories can be found at Universal Resource Locator http://bqs.usgs.gov/ precip/project_overview/frameil.htm. For magnesium, potassium, chloride, $\mathrm{pH}$, and specific conductance, the number of data points outside the control limits decreased from 1997 to 1999. Sodium and ammonium exhibit an upward trend in the number of data points outside the control limits during the same time period.

\section{Intralaboratory Bias}

Intralaboratory bias (bias within a single laboratory) is defined as a systematic difference between the measured and expected values arising from sample handling and analysis procedures within a single laboratory. Potential bias for laboratories participating in the interlaboratory-comparison program was evaluated by the following methods: (1) comparison of laboratory results to the certified values and the estimated uncertainties reported by HPS for standard reference material SP-97 and SP-98, and (2) comparison of laboratory results to those expected for Ultrapure deionized-water samples (table 4). 

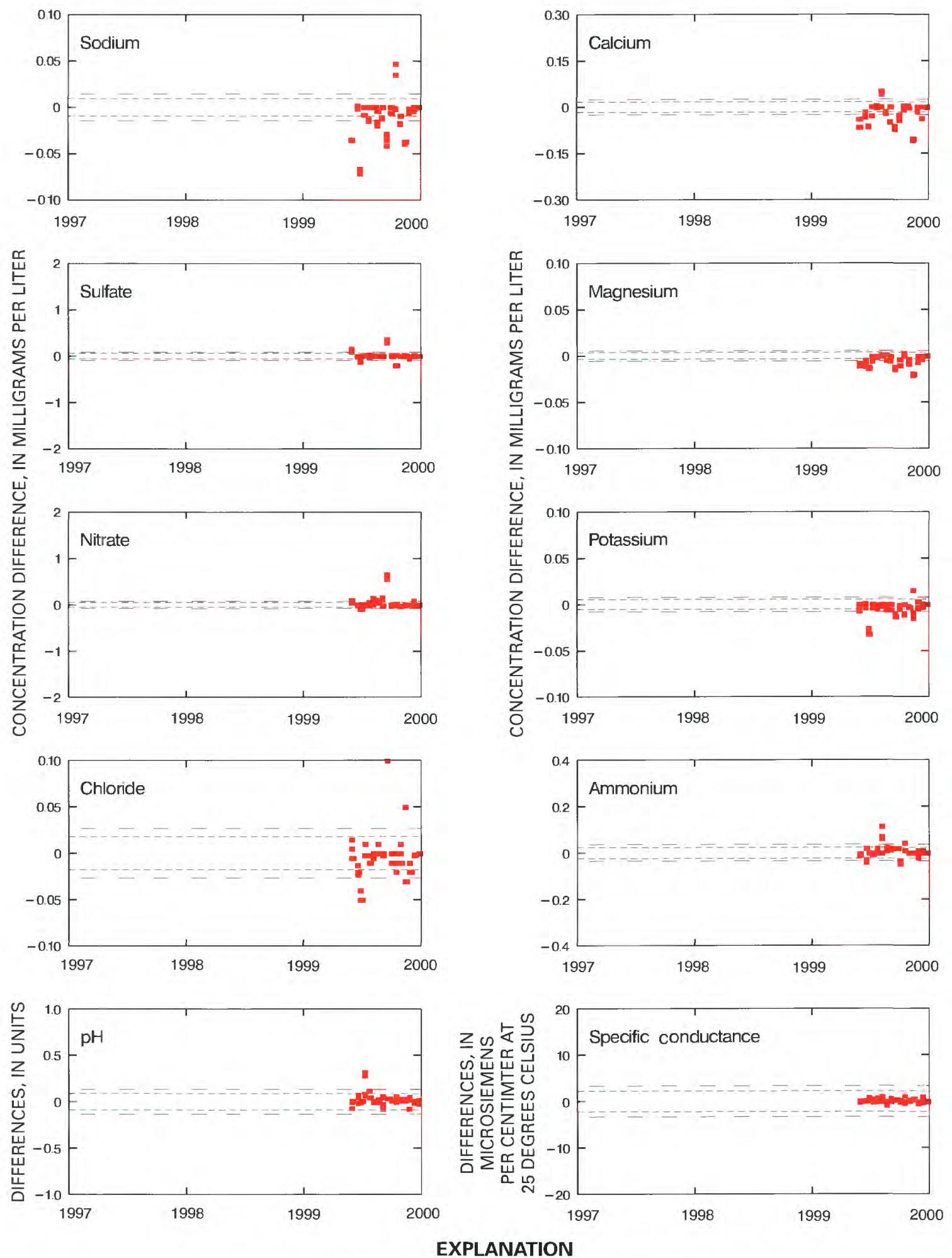

Warning limits ( +2 and $-2 \mathrm{~F}-\mathrm{pseudosigmas}$ from zero difference line)

Control limits ( +3 and -3 F-pseudosigmas from zero difference line)

Figure 24. Difference between the measured value reported by the Acid Deposition and Oxidant Research Center and the median value calculated for all participating laboratories in the interlaboratory-comparison program during 1997 through 1999. Samples reported as less than the detection limit (about 3 percent of the samples) were removed. 

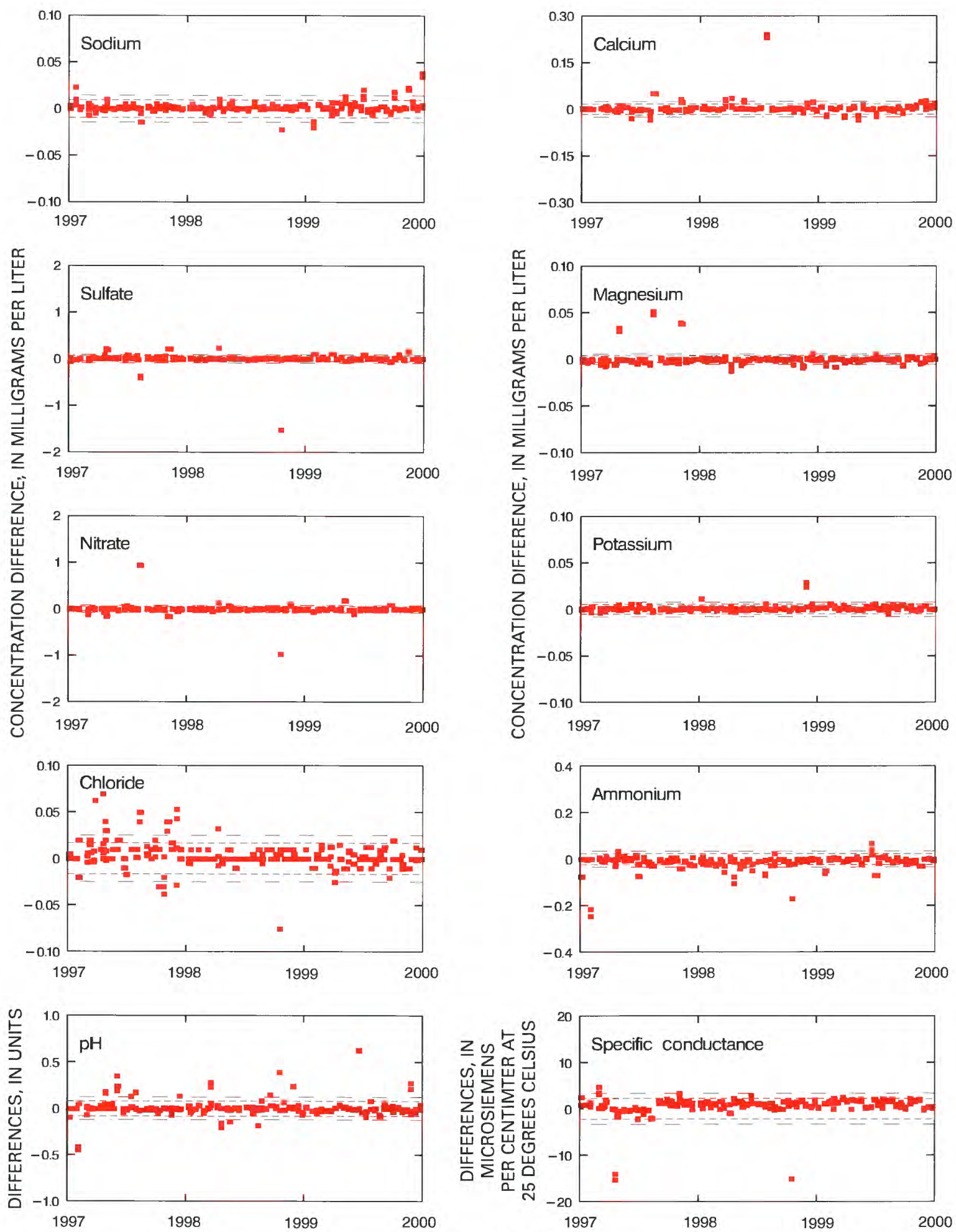

\section{EXPLANATION}

Warning limits $(+2$ and $-2 \mathrm{~F}-$ pseudosigmas from zero difference line)

- $\quad$ Control limits ( +3 and -3 F-pseudosigmas from zero difference line)

Figure 25. Difference between the measured value reported by the Illinois State Water Survey, Central Analytical Laboratory and the median value calculated for all participating laboratories in the interlaboratorycomparison program during 1997 through 1999. Samples reported as less than the detection limit (about 3 percent of the samples) were removed. 

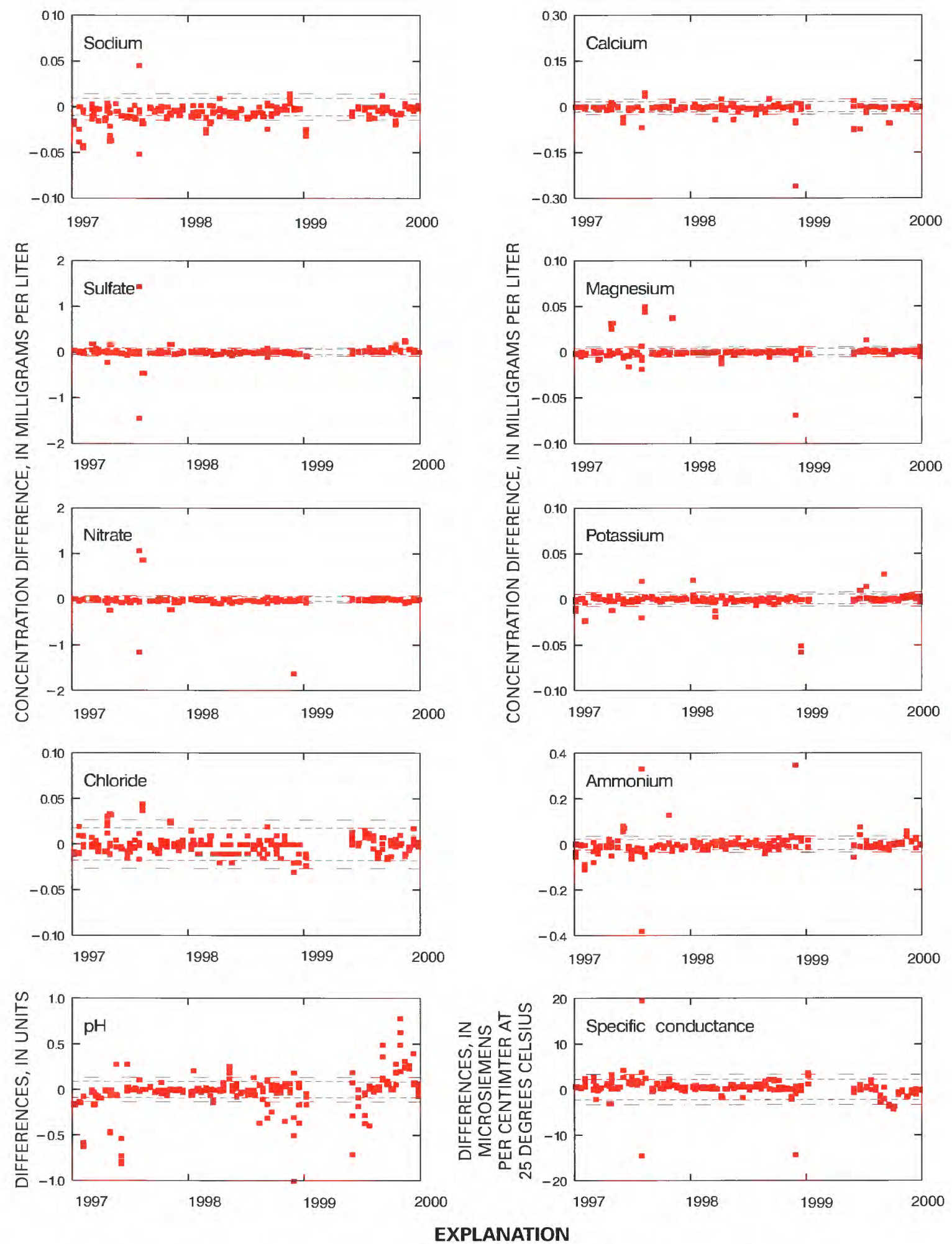

Warning limits ( +2 and -2 F-pseudosigmas from zero difference line)

Control limits ( +3 and -3 F-pseudosigmas from zero difference line)

Figure 26. Difference between the measured value reported by the Environmental Science and Engineering, Inc., Laboratory and the median value calculated for all participating laboratories in the interlaboratorycomparison program during 1997 through 1999. Samples reported as less than the detection limit (about 3 percent of the samples) were removed. 

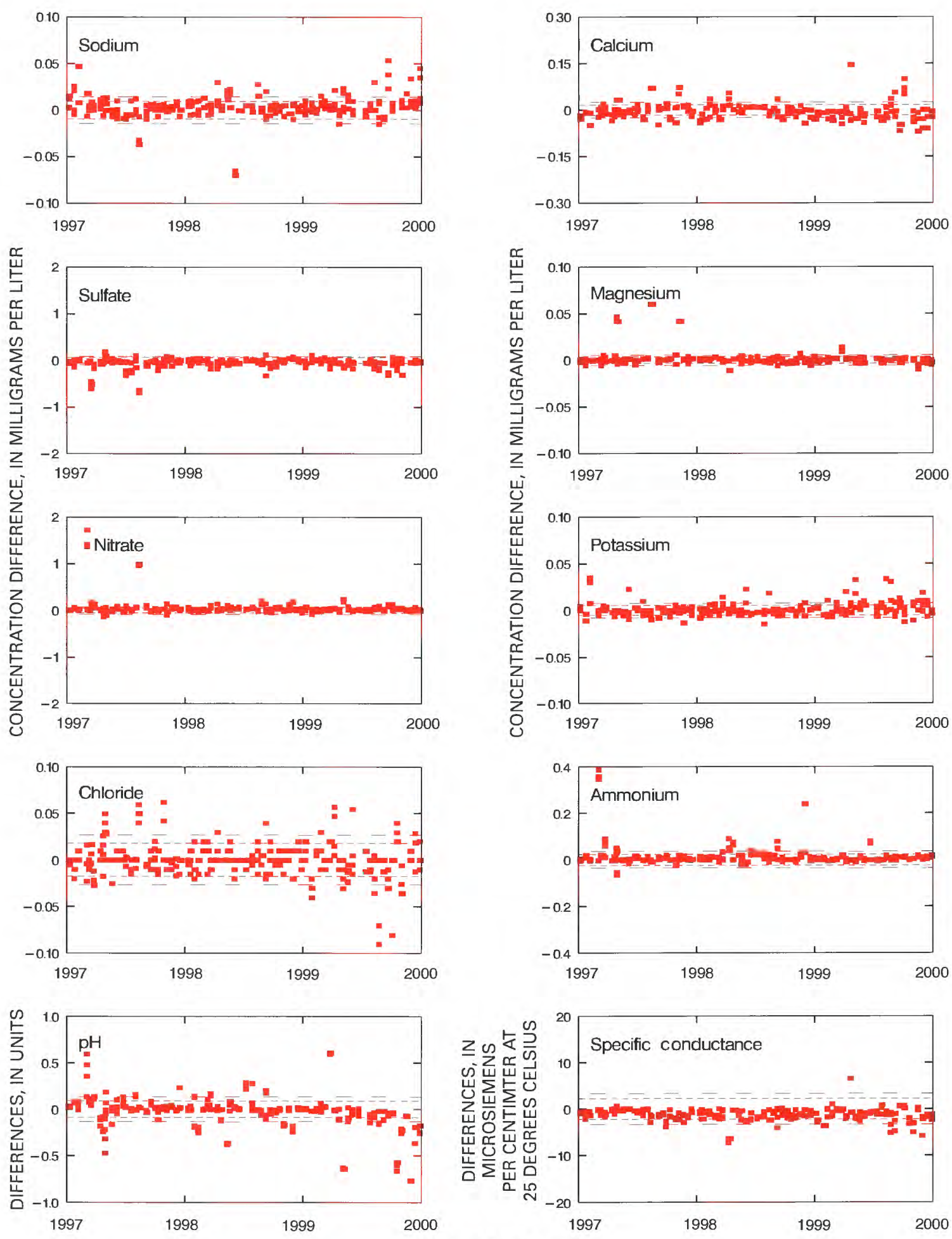

\section{EXPLANATION}

Warning limits (+2 and -2 F-pseudosigmas from zero difference line)

Control limits ( +3 and -3 F-pseudosigmas from zero difference line)

Figure 27. Difference between the measured value reported by the Ontario Ministry of the Environment, Dorset Research Facility in Ontario, Canada, and the median value calculated for all participating laboratories in the interlaboratory-comparison program during 1997 through 1999. Samples reported as less than the detection limit (about 3 percent of the samples) were removed. 

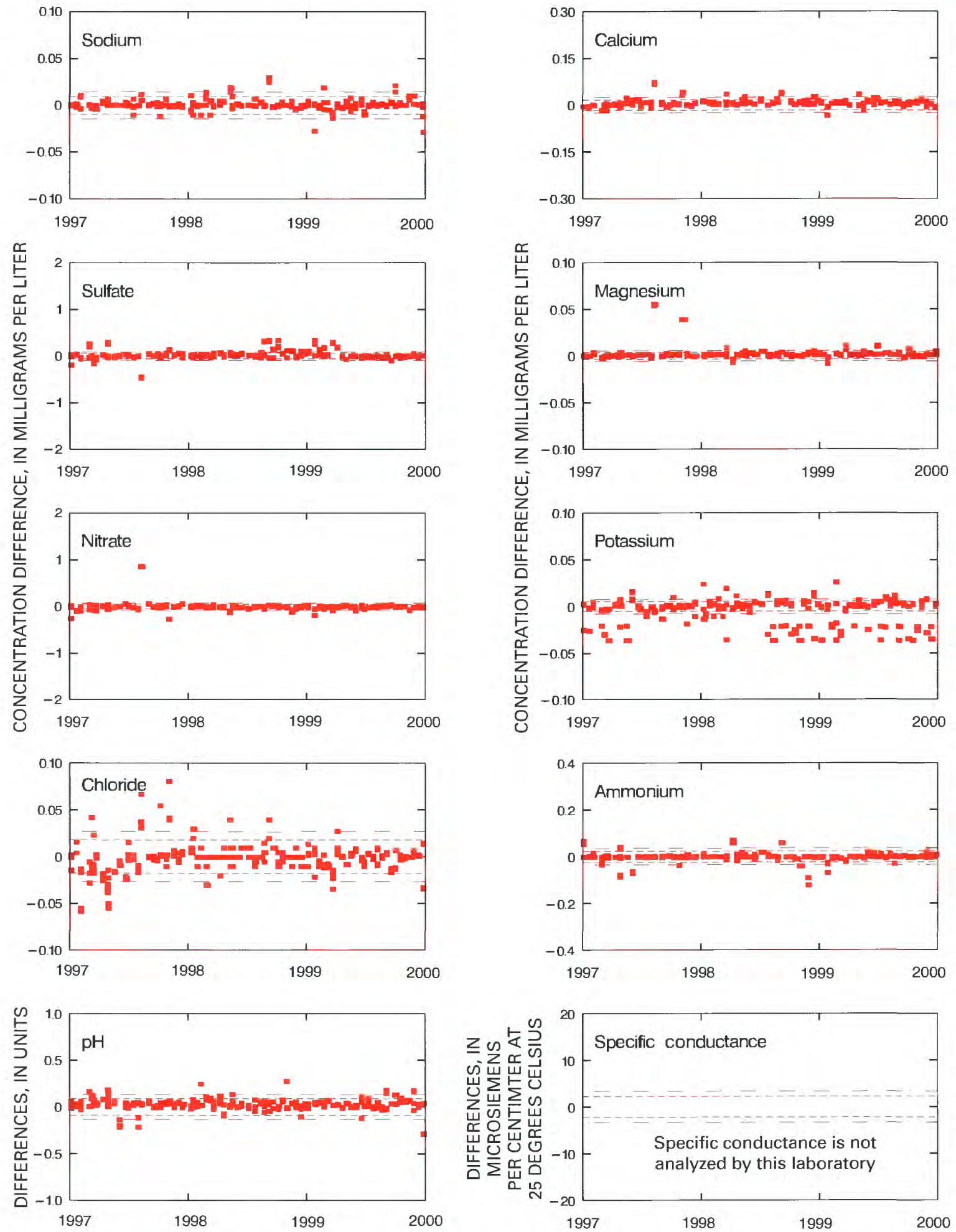

EXPLANATION

Warning limits ( +2 and -2 F-pseudosigmas from zero difference line)

- Control limits ( +3 and -3 F-pseudosigmas from zero difference line)

Figure 28. Difference between the measured value reported by the Meteorological Services of Canada and the median value calculated for all participating laboratories in the interlaboratory-comparison program during 1997 through 1999. Samples removed as less than the detection limit (about 3 percent of the samples) were removed. 

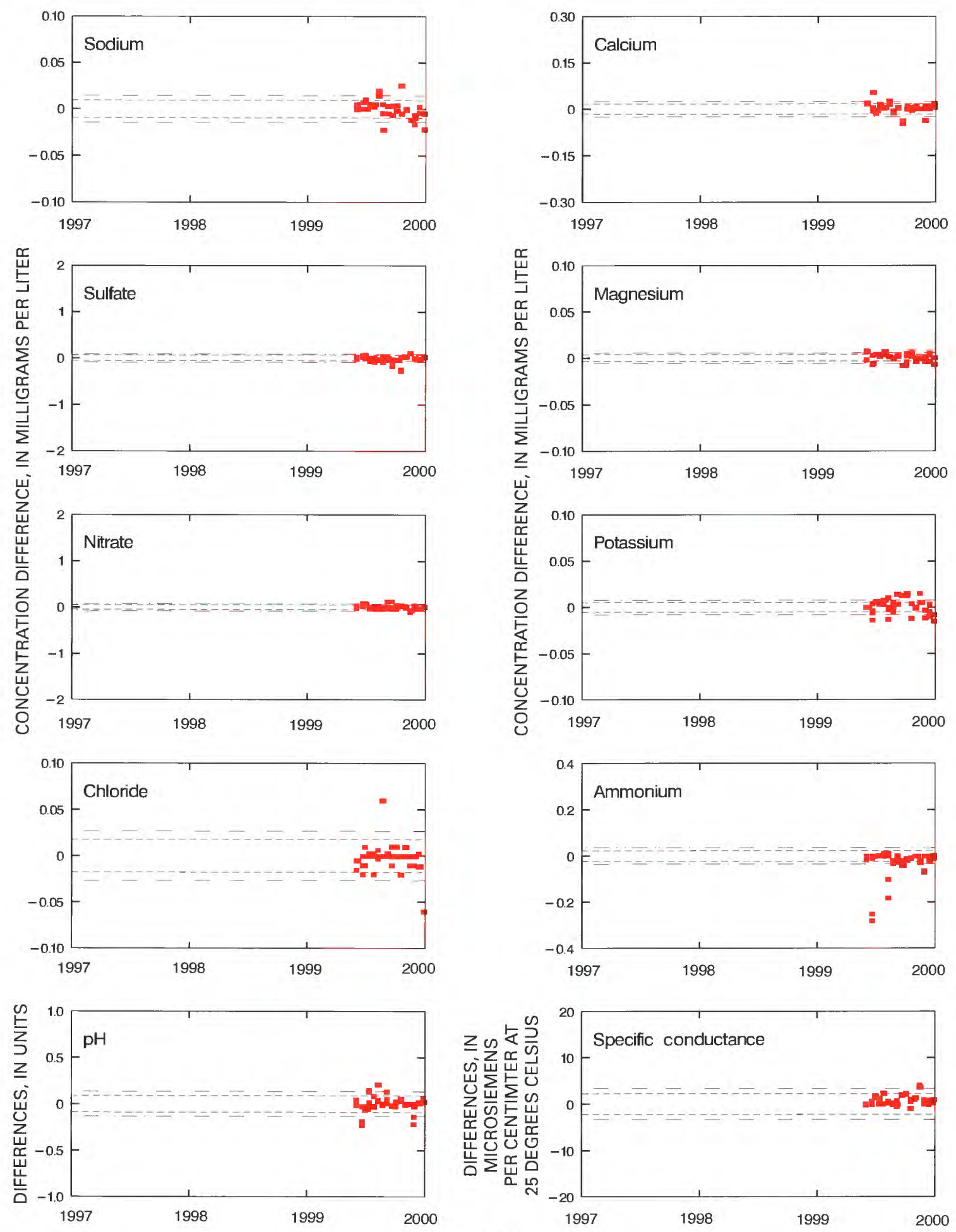

EXPLANATION

Warning limits $(+2$ and -2 F-pseudosigmas from zero difference line)

Control limits ( +3 and -3 F-pseudosigmas from zero difference line)

Figure 29. Difference between the measured value reported by the Norwegian Institute for Air Research and the median value calculated for all participating laboratories in the interlaboratory-comparison program during 1997 through 1999. Samples reported as less than the detection limit (about 3 percent of the samples) were removed. 

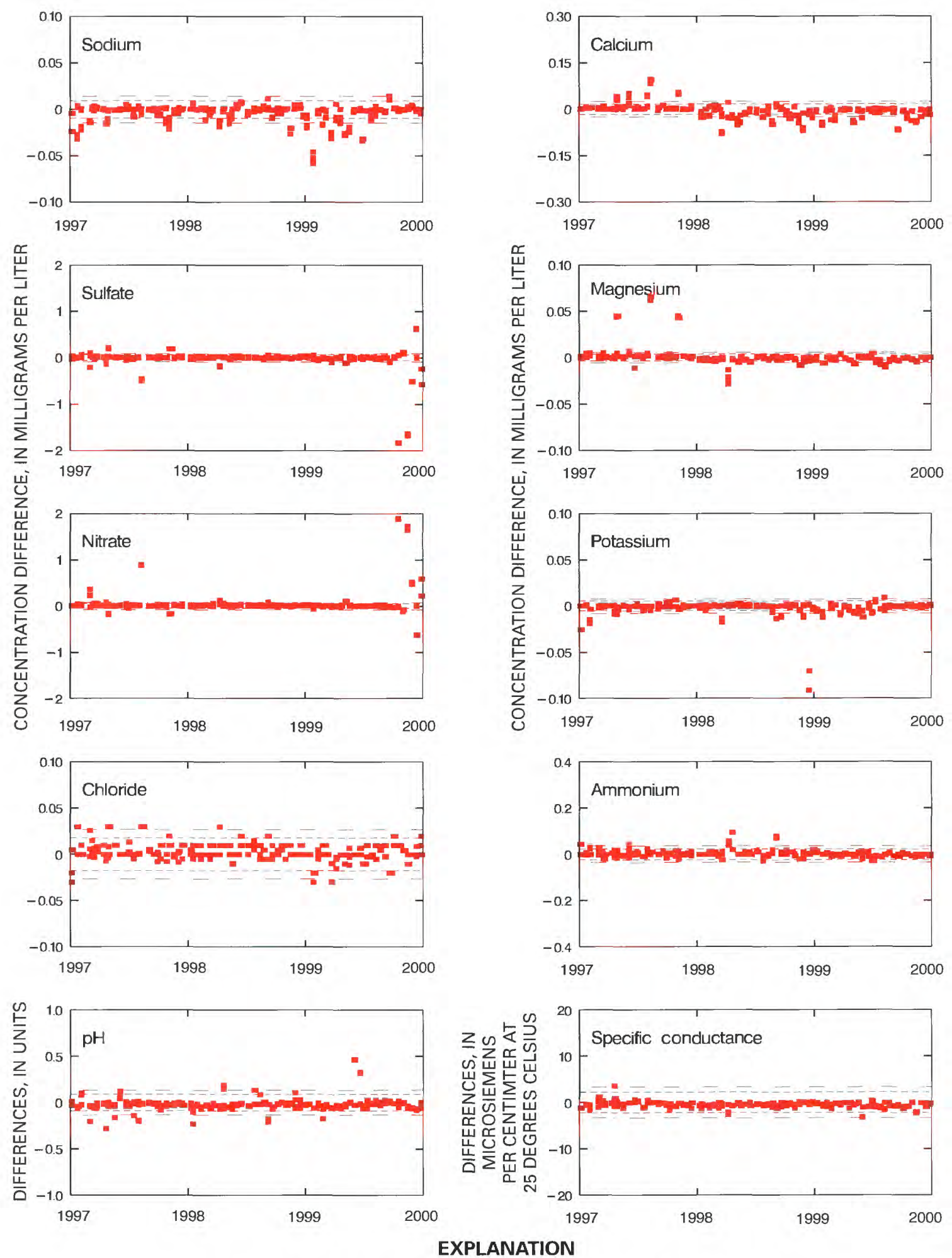

Warning limits ( +2 and -2 F-pseudosigmas from zero difference line)

Control limits $(+3$ and $-3 \mathrm{~F}$-pseudosigmas from zero difference line)

Figure 30. Difference between the measured value reported by the Shepard Analytical Services and the median value calculated for all participating laboratories in the interlaboratory-comparison program during 1997 through 1999. Samples reported as less than the detection limit (about 3 percent of the samples) were removed. 

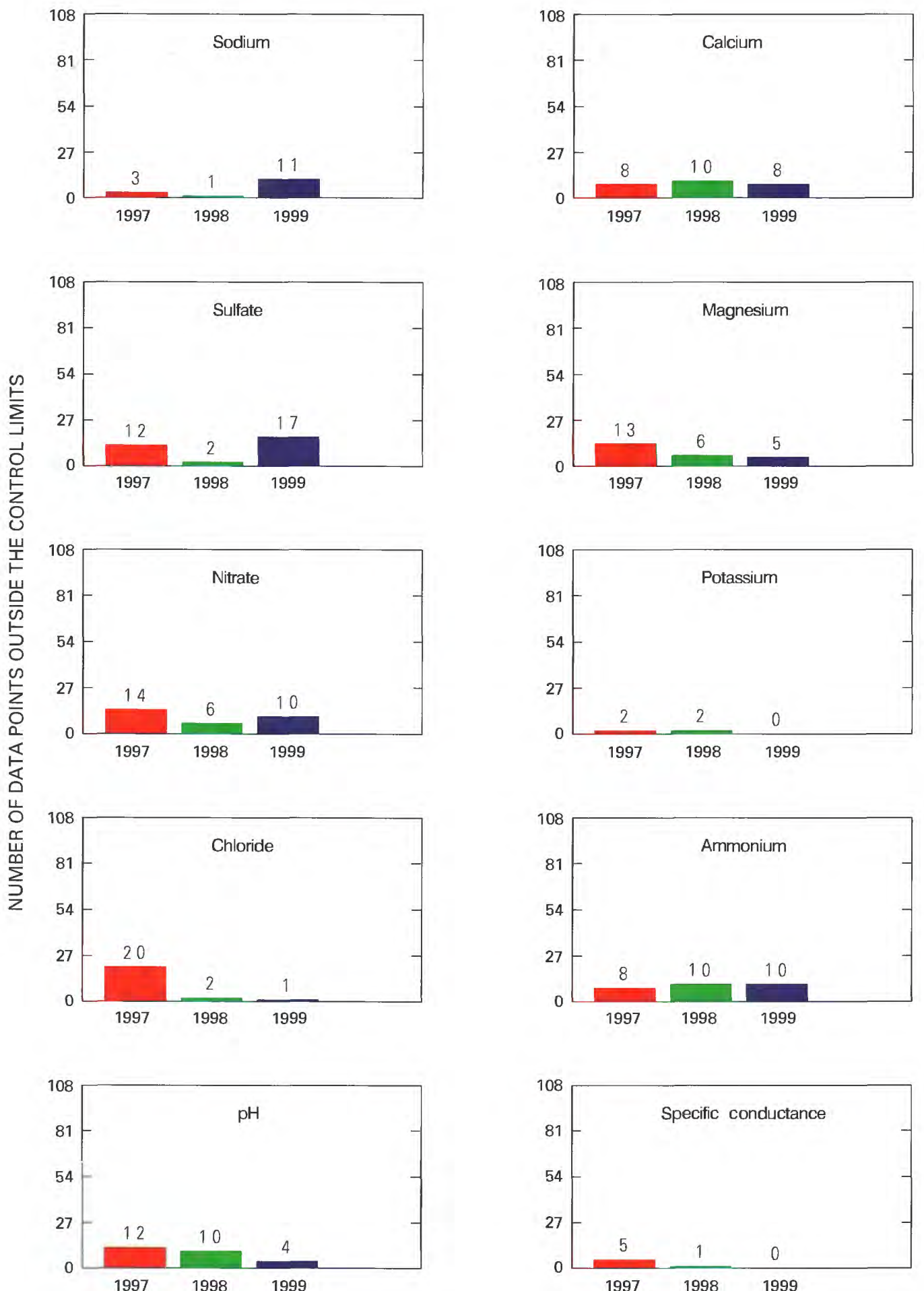

Figure 31. Graphs depicting the number of data points outside the control limits for the Illinois State Water Survey, Central Analytical Laboratory during 1997 through 1999. 


\section{Certified Standard Reference Samples}

HPS provides NIST-traceable certified ranges for the target values for all of the parameters measured in standard reference samples SP-97 and SP-98 except for $\mathrm{pH}$ and specific conductance. The certified ranges are extremely small and often are a single point value. The ability of a laboratory to achieve the extremely tight ranges set by the suppliers of the certified samples is an indication of outstanding precision. Lesser precision or bias could be indicated when the laboratory median values differ from the certified target values by 10 percent or more, an amount deemed potentially chemically significant. A summary of the median values for each laboratory and the analyte range reported by HPS for standard-reference materials SP-97 and SP-98 is presented in table 18. A summary of the estimated uncertainty ranges for the HPS standard-reference materials SP-97 and SP-98 and the median values for each laboratory are presented in table 18. The gray shading in table 18 indicates that the median value is outside the certified range for the given reference material. Orange shading indicates the median value differed from the certified range by 10 percent or more. Boxed areas indicate that a median value for a given analyte was calculated from less than 10 values (the maximum possible was 22 values). Negative numbers indicate the value was less than the laboratory's minimum reporting limit. Unshaded values for a given analyte were within the extremely tight ranges set by the suppliers of the certified values. The CAL met the NIST-traceable certified ranges more often than most of the other participating laboratories. Because the NIST-traceable certified ranges are so narrow, it is not of great concern for program objectives that many of the laboratories were only able to achieve these ranges for a few analytes. Median analyte values outside the range usually differed from the NIST-traceable certified range by only 0.01 to $0.05 \mathrm{mg} / \mathrm{L}$ for the major ions.

A more useful measure of laboratory performance was to determine how well laboratories could achieve median values within 10 percent of the NISTtraceable certified range. Although all 10 analytes (ammonium, calcium, chloride, nitrate, magnesium, potassium, $\mathrm{pH}$, sodium, specific conductance, and sulfate) are measured on the certified reference samples, certified expected values are provided for only 8 analytes. Certified values for $\mathrm{pH}$ and specific conductance are not provided. Between 1997 and
1999, CAL, MOE, MSC, and SA all had eight of eight median analyte values within the range set as the median of the certified expected value range of \pm 10 percent for the SP-97 solution. ESE had seven of eight median analyte values within the range set as the median of the certified expected value range of \pm 10 percent for the SP-97 solution, and NILU had six of eight and ADORC had four of eight median analyte values within the same acceptable range. However, because ADORC and NILU results are based on only 6 months of data as these laboratories only began participating in the study in June 1999, results for these laboratories may not be comparable to results for other laboratories. For the SP-98 solution, ADORC, MOE, and MSC all had seven of eight median values within the range set as the median of the certified expected value range \pm 10 percent for the SP-98 solution. CAL and ESE and SA each had six of eight median values within this range for the SP-98 solution, while NILU had five of eight median values within the acceptable range. The general similarity among laboratories in terms of their ability to achieve median values within 10 percent of the NIST-certified range also was noteworthy.

\section{Ultrapure Deionized-Water Samples}

In order to detect possible low-level sample contamination resulting from laboratory procedures, six Ultrapure deionized-water samples were included among the samples submitted to the participating laboratories throughout 1997; in 1998 and 1999. eight Ultrapure deionized-water samples were sent to each laboratory during each year. Table 19 shows the number of times each laboratory reported a concentration greater than the "standardized" reporting limit in a solution not expected to contain detectable analyte concentrations. In order to facilitate the comparison among laboratories using different minimum reporting limits, all data for a given ion less than the largest minimum reporting limit used by one of the seven participating laboratories were set equal to the largest minimum reporting limit. If the reporting limits were not standardized in this manner, a comparison among laboratories would be heavily influenced by differences in reporting limits. Laboratories with lower reporting limits would have a much higher incidence of "hits" for the deionized-water samples than laboratories with higher minimum reporting limits. 


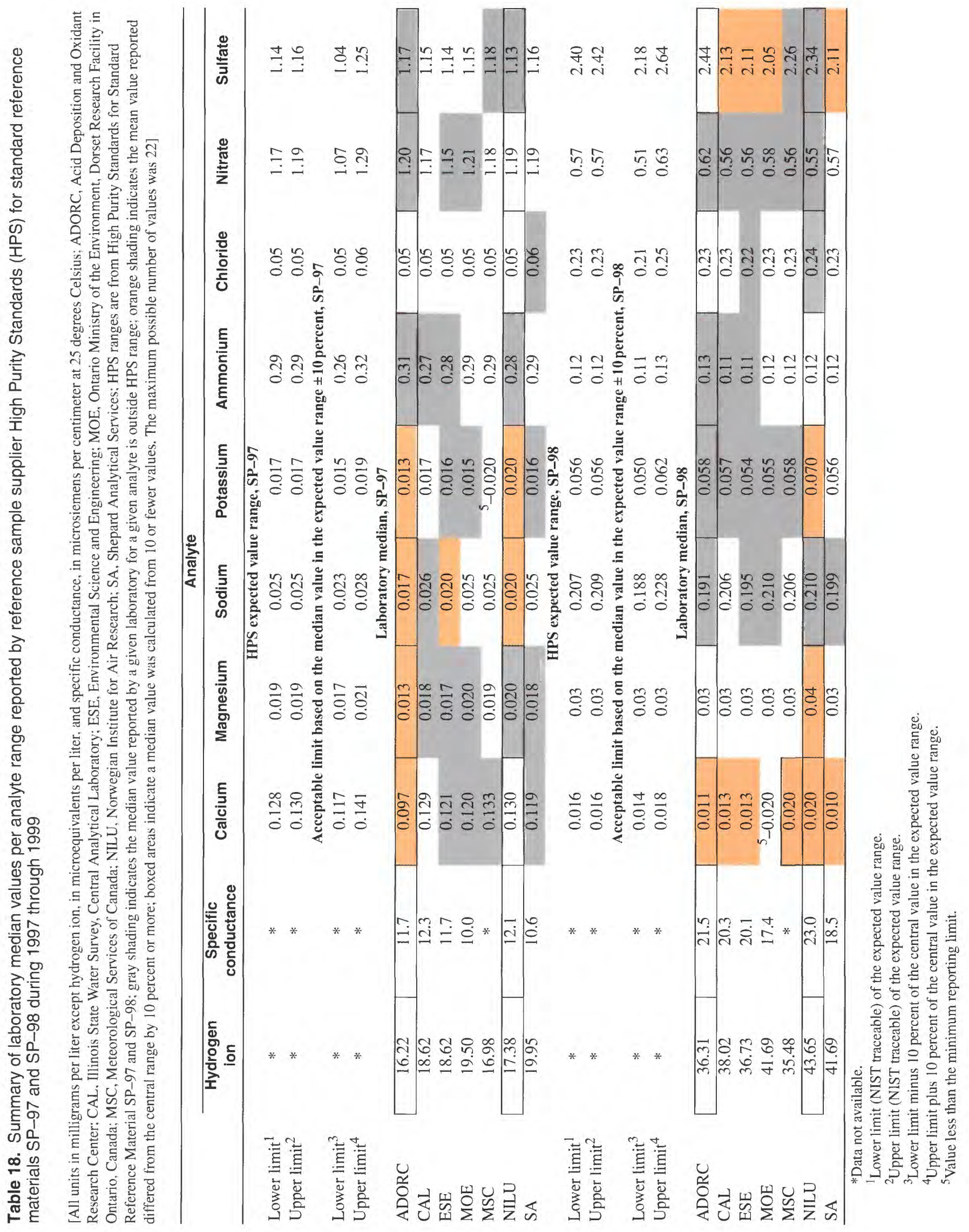


Table 19. Number of analyte determinations greater than the largest minimum reporting limit used by any participating laboratory for each ion for the Ultrapure deionized-water samples during 1997 through 1999

[ADORC, Acid Deposition and Oxidant Research Center; CAL, Illinois State Water Survey, Central Analytical Laboratory; ESE, Environmental Science and Engineering, Inc.; MOE, Ontario Ministry of the Environment, Dorset Research Facility in Ontario, Canada; MSC, Meteorological Services of Canada; NILU, Norwegian Institute for Air Research; SA, Shepard Analytical Services]

\begin{tabular}{lccccccc}
\hline \multicolumn{1}{c}{ Analyte } & ADORC & CAL & ESE & MOE & MSC & NILU & SA \\
\hline Calcium & 0 & 0 & 0 & 1 & 0 & 0 & 0 \\
Magnesium & 0 & 0 & 0 & 0 & 0 & 0 & 0 \\
Sodium & 0 & 0 & 1 & 3 & 0 & 0 & 0 \\
Potassium & 0 & 0 & 1 & 3 & 1 & 0 & 0 \\
Ammonium & 0 & 0 & 7 & 0 & 0 & 0 & 0 \\
Chloride & 0 & 0 & 0 & 0 & 0 & 0 & 0 \\
Nitrate & 0 & 0 & 0 & 0 & 0 & 0 & 0 \\
Sulfate & 0 & 0 & 0 & 0 & 0 & 0 & 0 \\
\hline
\end{tabular}

ESE reported one value greater than the "standardized" reporting limit for sodium and potassium and seven values greater than the "standardized" reporting limit for ammonium during 1997-99. MOE reported one value greater than the "standardized" reporting limit for calcium and three values greater than the "standardized" reporting limit for sodium and for potassium during the same time period. MSC reported one value greater than the "standardized" reporting limit for potassium during 1997-99. None of the other laboratories reported any ion values greater than the "standardized" reporting limit in any of the years.

\section{Laboratory Precision}

The precision of an estimator is defined as a measure of the repeatability of the estimator (Ostle and Malone, 1988). Therefore, precision may be expressed in terms of the variance, with large variance signifying a lack of precision (Ostle and Malone, 1988). One method of evaluating variance is to determine the extent to which a given set of measurements of the same sample agrees with its most probable value (that is, the median, mean, or certified target value). For this analysis, laboratory precision was estimated for each analyte by calculating the 50th and 90th percentiles of the absolute differences for the results reported for the replicate natural and synthetic wet-deposition samples (table 20). Differences were calculated from 26 sample pairs of replicate natural samples per year for each laboratory, with the exception of ESE, ADORC, and NILU. ESE received only 18 of the 26 sample pairs during 1999. ADORC and NILU received 16 of the 26 sample pairs during 1999 and did not receive any during 1997-98. Analyte concentrations reported as less than the MRL were set equal to one-half the MRL.

Cation precision estimates at the 50th percentile were similar among laboratories. All participating laboratories had median absolute differences that were less than or equal to $0.005 \mathrm{mg} / \mathrm{L}$ for the cations. Minor differences among laboratories were more readily apparent for precision estimates at the 90th percentile for calcium, sodium, potassium, and ammonium. For example, sodium precision estimates at the 90th percentile during 1997-99 varied about an order of magnitude from $0.003 \mathrm{mg} / \mathrm{L}$ for the laboratory with the least measurement variability for sodium to $0.025 \mathrm{mg} / \mathrm{L}$ for the laboratory with the highest variability. Even though the 90th percentile values sometimes varied by about an order of magnitude, the largest 90th percentile values were still considered relatively small numbers at no more than a few multiples of the minimum reporting limit. While anion precision estimates at the 50th percentile exhibited greater variability among laboratories than cation precision estimates at the 50th percentile, all of the participating laboratories had median absolute differences that were less than or equal to $0.050 \mathrm{mg} / \mathrm{L}$ for the anions.

The $\mathrm{pH}$ (expressed as hydrogen ion) precision estimates at both the 50th and 90th percentiles exhibited only minor variability among laboratories. Median absolute differences ranged from 0.000 microequivalents per liter $(\mu \mathrm{eq} / \mathrm{L})$ to $1.039 \mu \mathrm{eq} / \mathrm{L}$; precision estimates at the 90 th percentile ranged from $0.818 \mu \mathrm{eq} / \mathrm{L}$ to $5.524 \mu \mathrm{eq} / \mathrm{L}$.

Six laboratories routinely reported specificconductance results during 1997-99. The MSC does not routinely report specific-conductance measurements. There was little variability among laboratories in their reported specific-conductance measurements; the median differences ranged from 0.00 to 0.30 microsiemens per centimeter $(\mu \mathrm{S} / \mathrm{cm})$. The 90 th percentile values ranged from 0.20 to $1.60 \mu \mathrm{S} / \mathrm{cm}$ during this same time period. 


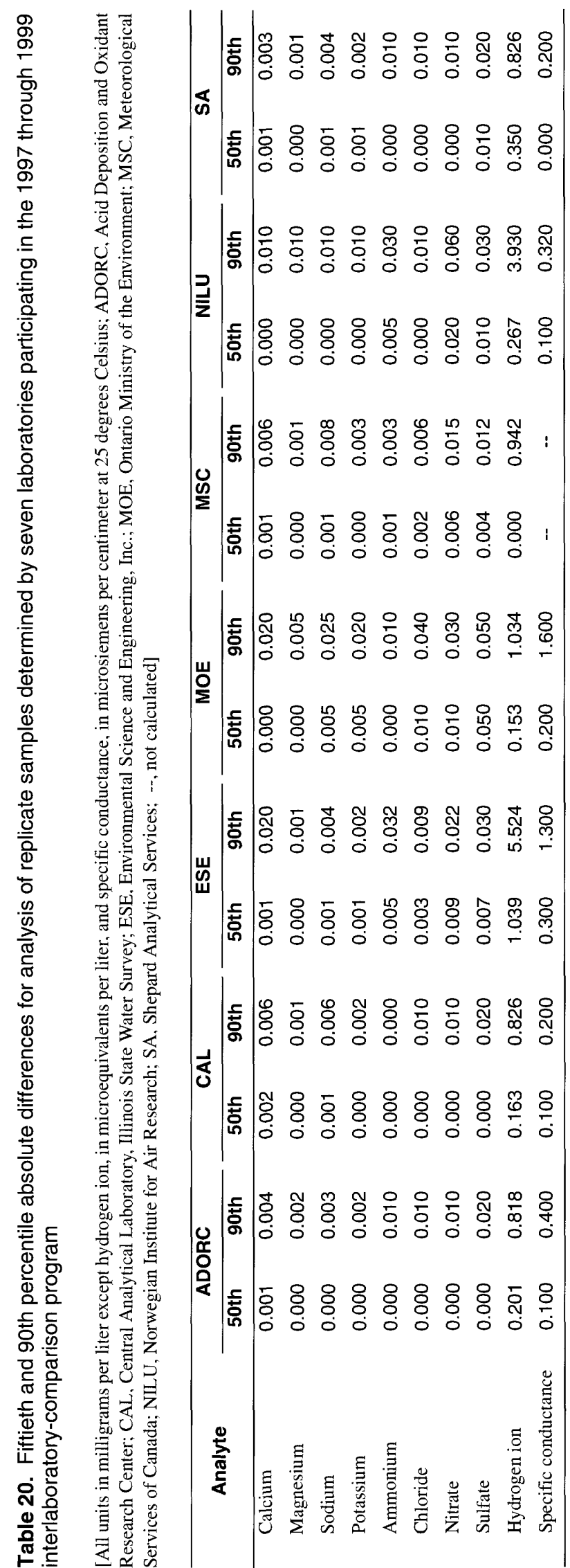




\section{COLLOCATED-SAMPLER PROGRAM}

In October 1988, the collocated-sampler program was established to provide a method of estimating the overall bias and precision of the precipitation-monitoring system used by the NADP/NTN. Included in this estimate of NADP/NTN precision is the variability from the point of sample collection through storage of the data in the database (Gordon, 1999). Nilles and others (1991) provide a detailed description of the collocated-sampler program. Since 1988, collocated sites have been operated on a water-year basis every year except 1994 (Gordon, 1999).

Beginning in 1997, the number of sites selected for the collocated-sampler program was reduced from four to two sites each year so funds would be available for the new field-audit program. The two sites selected for the collocated-sampler program in water year 1997-October 1, 1996, through September 30, 1997-were FL14 (Quincy) and OR09 (Silver Lake Ranger Station). In water year 1998, the two sites selected for participation in the collocated-sampler program were VA28 (Shenandoah National Park) and WY95 (Brooklyn Lake). In water year 1999, the two sites selected for participation were MA08 (Quabbin Reservoir) and MN01 (Cedar Creek). The locations of sites participating in the collocated-sampler program during 1997 through 1999 are shown in figure 32.

NADP/NTN guidelines for site selection and installation (Bigelow, 1984) are used in the establishment of each collocated site. Site selection is made with the goal of distributing sites among diverse regional locations, ecoregions, and precipitation regimes. In an effort to minimize data loss due to changes in personnel, sites with stable operational histories are given priority consideration. Sites are not considered if there is a lack of space for collocated equipment unless creative solutions, such as moving or rebuilding the perimeter fencing, can be implemented.

Following site selection, equipment for the collocated-sampler program was shipped and installed by USGS personnel. At some locations, the site operator assisted with the installation of equipment. The new collocated site is designed to replicate the existing site in detail to the fullest extent possible. For example, if the existing equipment is located on platforms, the new equipment is installed on platforms as well. The equipment also was field tested by USGS personnel to ensure that the equipment was in good working order before samples were collected at the new sites. The site operator processed samples from each pair of collectors using standard NADP/NTN procedures (Bigelow and Dossett, 1988). Site operators were given the option of forgoing onsite $\mathrm{pH}$ and specific-conductance measurements of samples from the newly installed collocated samplers. Regardless of whether the $\mathrm{pH}$ and specificconductance measurements were made, a $20-\mathrm{mL}$ aliquot was removed from samples with volumes greater than $70 \mathrm{~mL}$ to ensure equivalent handling of both samples from the collocated-sampler site. The CAL analyzed samples from the collocated sites as if they were routine weekly NADP/NTN samples.

Data from the original and collocated equipment were analyzed in two ways. For the purpose of site characterization (that is, determining the median sample chemistry or median precipitation) the data from the original and collocated sites were pooled. For the purpose of comparing an original and collocated site, the data from the original and collocated sites were analyzed for differences. Data from the original and collocated site are formally referred to by the fourcharacter site code of the original site followed by the four-character site code of the collocated site. For example, the Quincy, Fla., site is formally referred to as FL14/14FL. For this analysis, the data used were from wet-deposition samples with volumes greater than $35 \mathrm{~mL}$ (laboratory type "W") that did not require dilution. Samples requiring dilution are inherently prone to greater analytical error. Median concentrations for selected analytes in weekly samples collected at the 1997-99 collocated sites are depicted in figure 33 . Figure 34 depicts the median hydrogen-ion concentration, median specific conductance, median sample volume, and median precipitation depth for these collocated sites.

Because the collocated sites have diverse climates, anthropogenic influences, and wetdeposition regimes, differences in their sample chemistry are expected. While median sample chemistry was often quite similar among the 1997-99 collocated sites, there were some notable differences. For example, Cedar Creek, Minn. (MN01/01MN), had larger median concentrations for calcium, magnesium, ammonium, and nitrate compared to the other collocated sites. The median annual chloride and sodium concentrations were approximately four times higher 


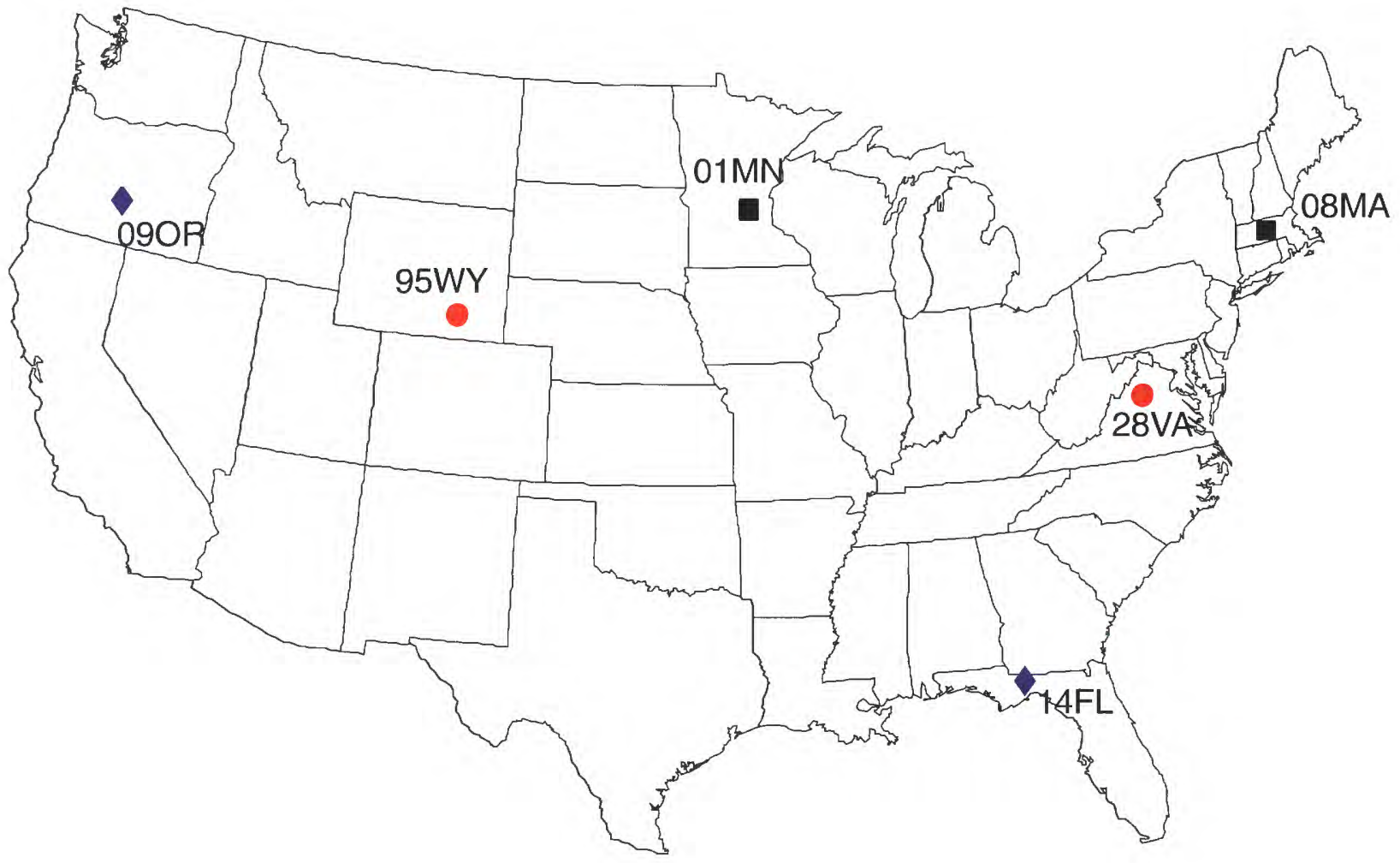

EXPLANATION

Collocated-sampler sites for water year 1997

\begin{tabular}{ccl}
$\begin{array}{c}\text { Collocated } \\
\text { Site Code }\end{array}$ & $\begin{array}{c}\text { Original } \\
\text { Site Code }\end{array}$ & Site Name \\
\hline $14 \mathrm{FL}$ & FL14 & QUINCY, FLORIDA \\
09OR & OR09 & SILVER LAKE RANGER STATION, OREGON
\end{tabular}

Collocated-sampler sites for water year 1998

Collocated Original

Site Code Site Code Site Name

\begin{tabular}{lll}
\hline 28VA & VA28 & SHENANDOAH NATIONAL PARK, VIRGINIA \\
95WY & WY95 & BROOKLYN LAKE, WYOMING
\end{tabular}

Collocated-sampler sites for water year 1999

\begin{tabular}{ccc}
$\begin{array}{c}\text { Collocated } \\
\text { Site Code }\end{array}$ & $\begin{array}{c}\text { Original } \\
\text { Site Code }\end{array}$ & Site Name \\
\hline 08MA & MA08 & QUABBIN RESERVOIR, MASSACHUSETTS \\
01MN & MN01 & CEDAR CREEK, MINNESOTA
\end{tabular}

Figure 32. Map showing location of National Atmospheric Deposition Program/National Trends Network sites with collocated samplers in water years 1997 through 1999. 

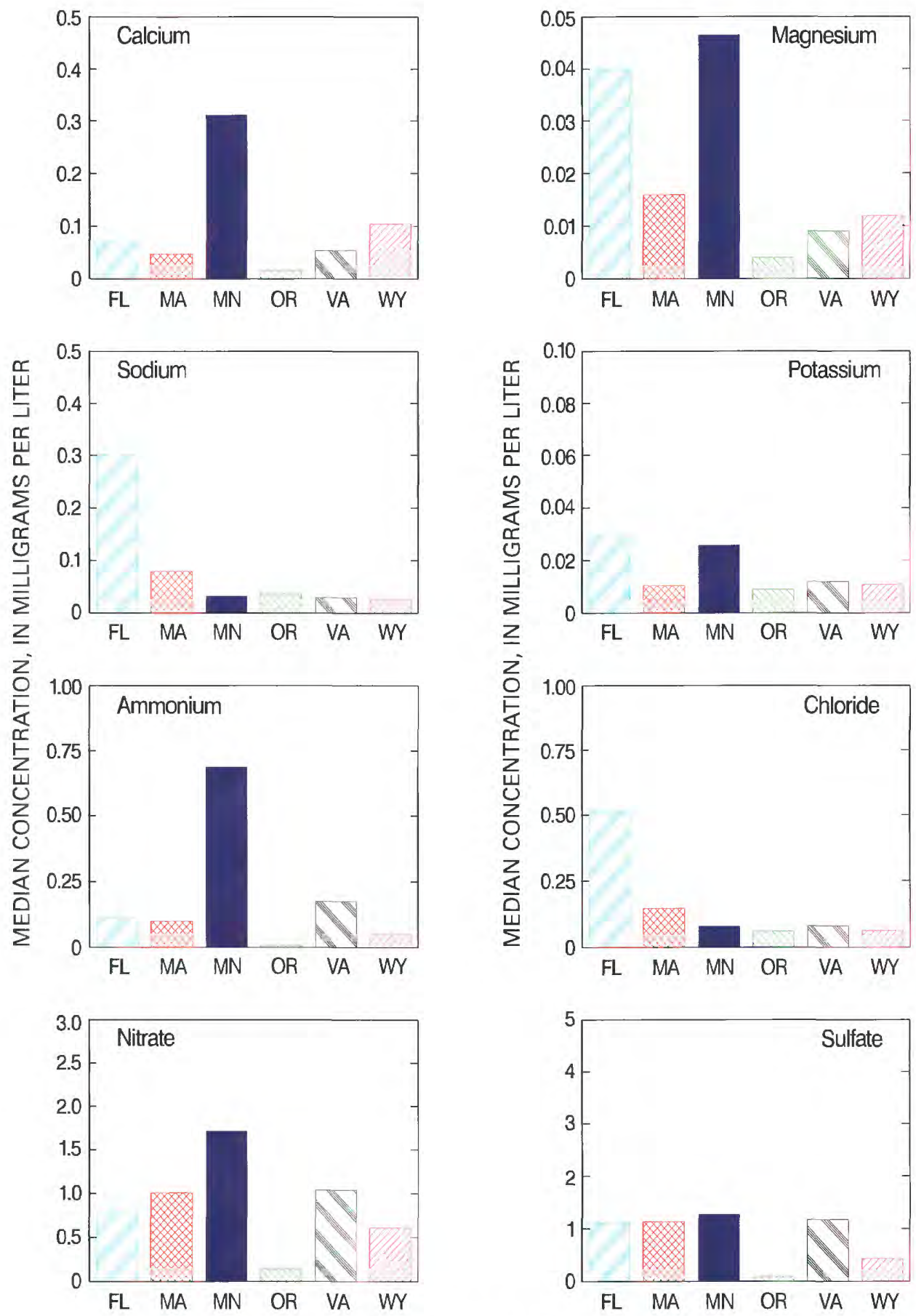

FL MA MN OR VA WY

\section{EXPLANATION}

Samples from sites FL14 and 14FL combined

Samples from sites MA08 and 08MA combined

Samples from sites MN01 and 01MN combined

Samples from sites OR09 and O9OR combined

Samples from sites VA28 and 28VA combined

Samples from sites WY95 and 95WY combined

Figure 33. Median concentrations for selected analytes in weekly samples collected at six National Atmospheric Deposition Program/National Trends Network sites with collocated samplers during 1997 through 1999. 

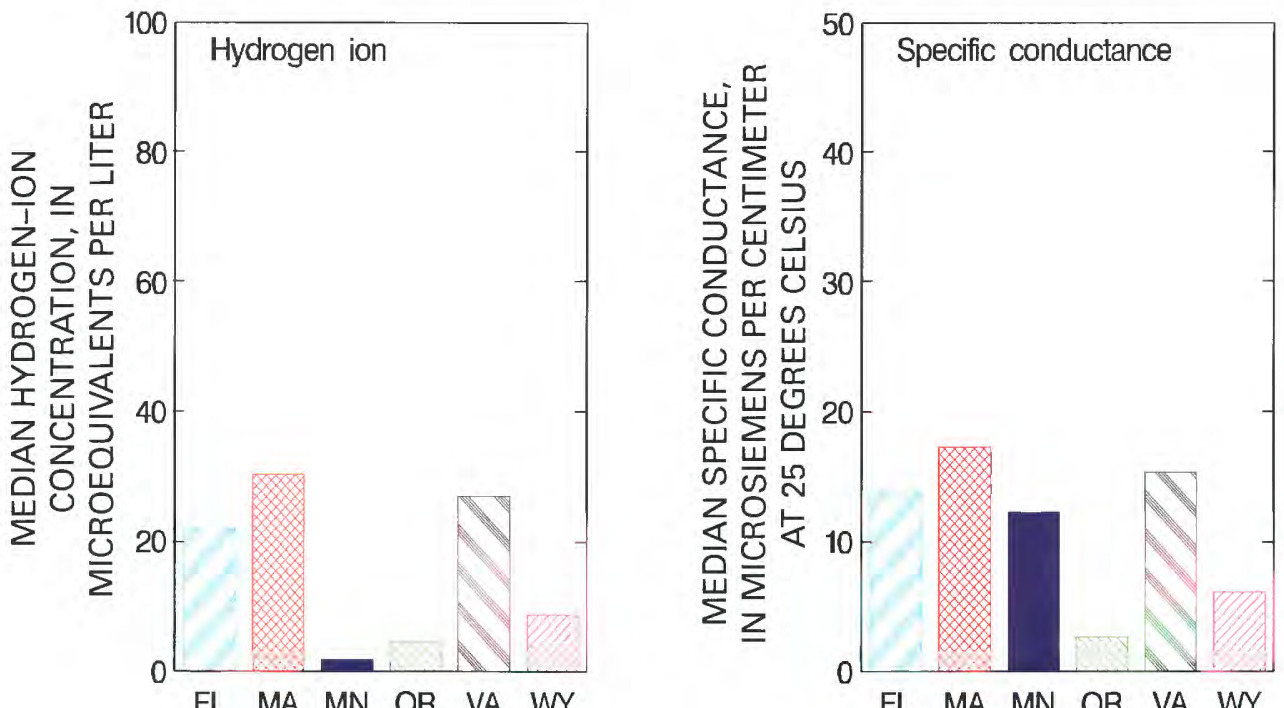

FL MA MN OR VA WY
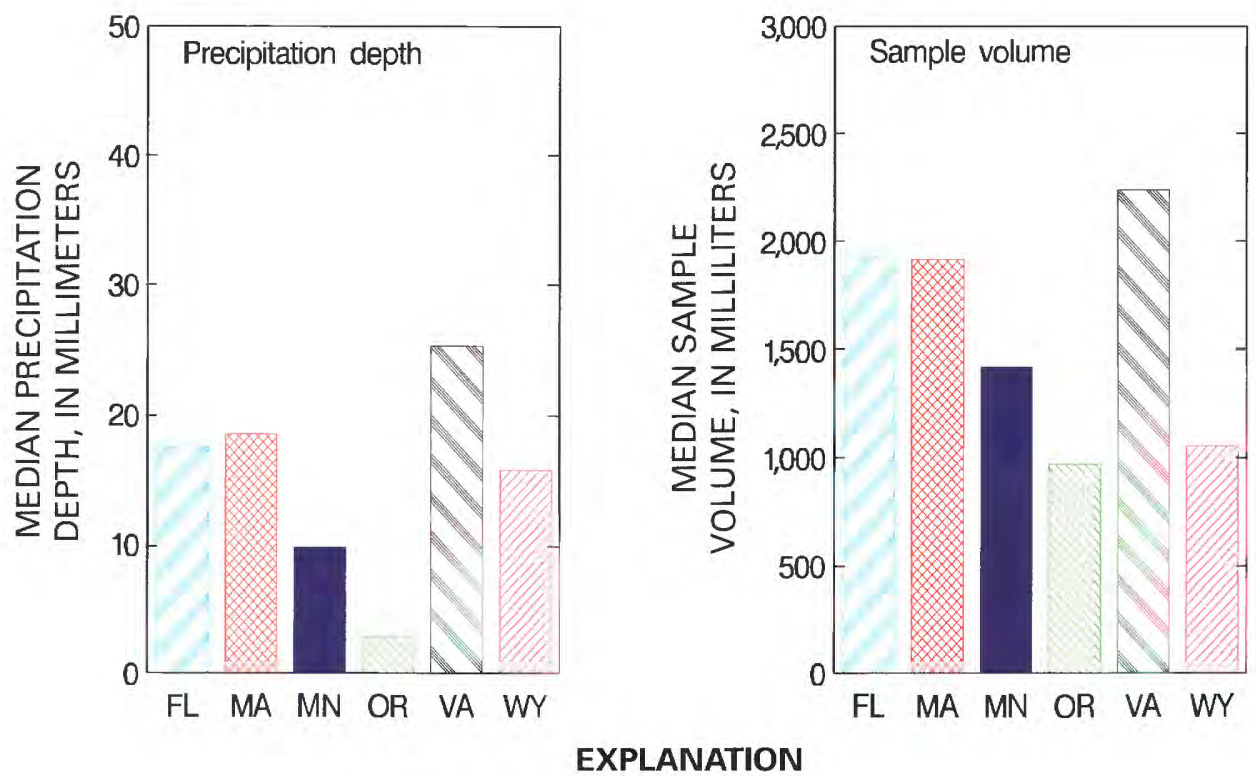

\section{EXPLANATION}

Samples from sites FL14 and 14FL combined

Samples from sites MA08 and 08MA combined

Samples from sites MN01 and 01MN combined

Samples from sites OR09 and 09OR combined

Samples from sites VA28 and 28VA combined

Samples from sites WY95 and 95WY combined

Figure 34. Median hydrogen-ion concentration, specific conductance, sample volume, and precipitation depth at National Atmospheric Deposition Program/National Trends Network sites with collocated samplers during 1997 through 1999. 
at the one marine-influenced sampling site, Quincy, Fla. (FL14/14FL), than at the other inland sampling sites. The median chloride and sodium concentrations at the five inland sites were similar. VA28 is close to the eastern urban corridor extending from New York to Washington, D.C., and is downwind from major sulfate and nitrate emission sources in the Midwest. Therefore, the VA28 collocated site recorded higher median nitrate and sulfate concentrations than were measured at the FL14, OR09, or WY95 collocated sites. The remote and semiarid site at Silver Lake Ranger Station in Oregon (OR09) had much lower median concentrations of calcium, ammonium, nitrate, and sulfate than the other sites. The median hydrogenion concentrations at VA28/28VA and MA08/08MA were more than five times greater than the median hydrogen-ion concentration at OR09/09OR.

Because annual summaries of NADP/NTN data describe precipitation chemistry in units of concentration and deposition (National Atmospheric Deposition Program, 2000), statistical summaries for both the concentration and deposition of ionic constituents are provided in this report. The weekly precipitation depth associated with each Belfort recording rain gage was used to calculate deposition values at the collocated sites. Concentration in milligrams per liter is multiplied by $10^{-1}$ times the rainfall depth in centimeters to yield deposition in kilograms per hectare. The variability in deposition amounts due to differences in rain-gage collection efficiency at collocated sites provides an estimate of the variability in deposition amounts at other NADP/NTN sites.

\section{Analytical Bias and Precision}

Bias and precision estimates for analytical data from each site were calculated from the median relative error and median absolute differences between the pairs of collocated samplers. The equations used to estimate median relative error from collocated data are:

$$
\begin{gathered}
\text { Relative error }= \\
{\left[\left(\mathrm{C}_{1}-\mathrm{C}_{2}\right) /\left(\mathrm{C}_{1}+\mathrm{C}_{2}\right) / 2\right] \times 100} \\
\text { Median relative error }(\mathrm{MRE})= \\
\mathrm{M}\left\{\left[\left(\mathrm{C}_{1}-\mathrm{C}_{2}\right) /\left(\mathrm{C}_{1}+\mathrm{C}_{2}\right) / 2\right]\right\} \times 100
\end{gathered}
$$

The equations used to estimate median absolute difference from collocated data are:

Absolute difference between collectors $=$

$$
\left|\mathrm{C}_{1}-\mathrm{C}_{2}\right|
$$

Median absolute difference $(\mathrm{MAD})=$

$$
\mathrm{M}\left\{\left|\left[\left(\mathrm{C}_{1}-\mathrm{C}_{2}\right) /\left(\mathrm{C}_{1}+\mathrm{C}_{2}\right) / 2\right]\right|\right\} \times 100
$$

where

$\mathrm{M}=$ median of all paired differences from equation 5, in milligrams per liter or kilograms per hectare;

$\mathrm{C}_{1}$ = sample concentration, in milligrams per liter, from the original precipitation sampler, or deposition, in kilograms per hectare, from the original precipitation sampler and rain gage; and

$\mathrm{C}_{2}$ = sample concentration, in milligrams per liter, from the collocated precipitation sampler, or deposition, in kilograms per hectare, from the collocated precipitation sampler and rain gage.

Bias was evaluated for each site and analyte by determining the bias or median relative error (equation 6) between collocated-sample concentrations (fig. 35 and table 21). Bias estimates for sample volume from the precipitation collectors and precipitation depth from the recording rain gages also were calculated (fig. 35). Median relative error expressed as a percentage for analyte concentrations and physical parameters from weekly collocated precipitation samples and replicate samples in 1997, 1998, and 1999 are shown in table 21 on both a concentration and deposition basis. Because the collocated paired samples were shipped from the sites weekly to the same laboratory at the same time, bias in the data set pairs is attributed to systematic differences in (1) raingage response, (2) sample collection, and (3) sample handling before shipment. Bias was within a range of $0 \pm 5$ percent for chloride, sulfate, hydrogen ion, specific conductance, and sample volume for all of the collocated sites. For calcium and sodium, bias rarely exceeded the range of $0 \pm 5$ percent. The general absence of bias as a significant contributor to overall variability in NADP/NTN wet-chemistry measurements from the six sites reflects the good precision and 

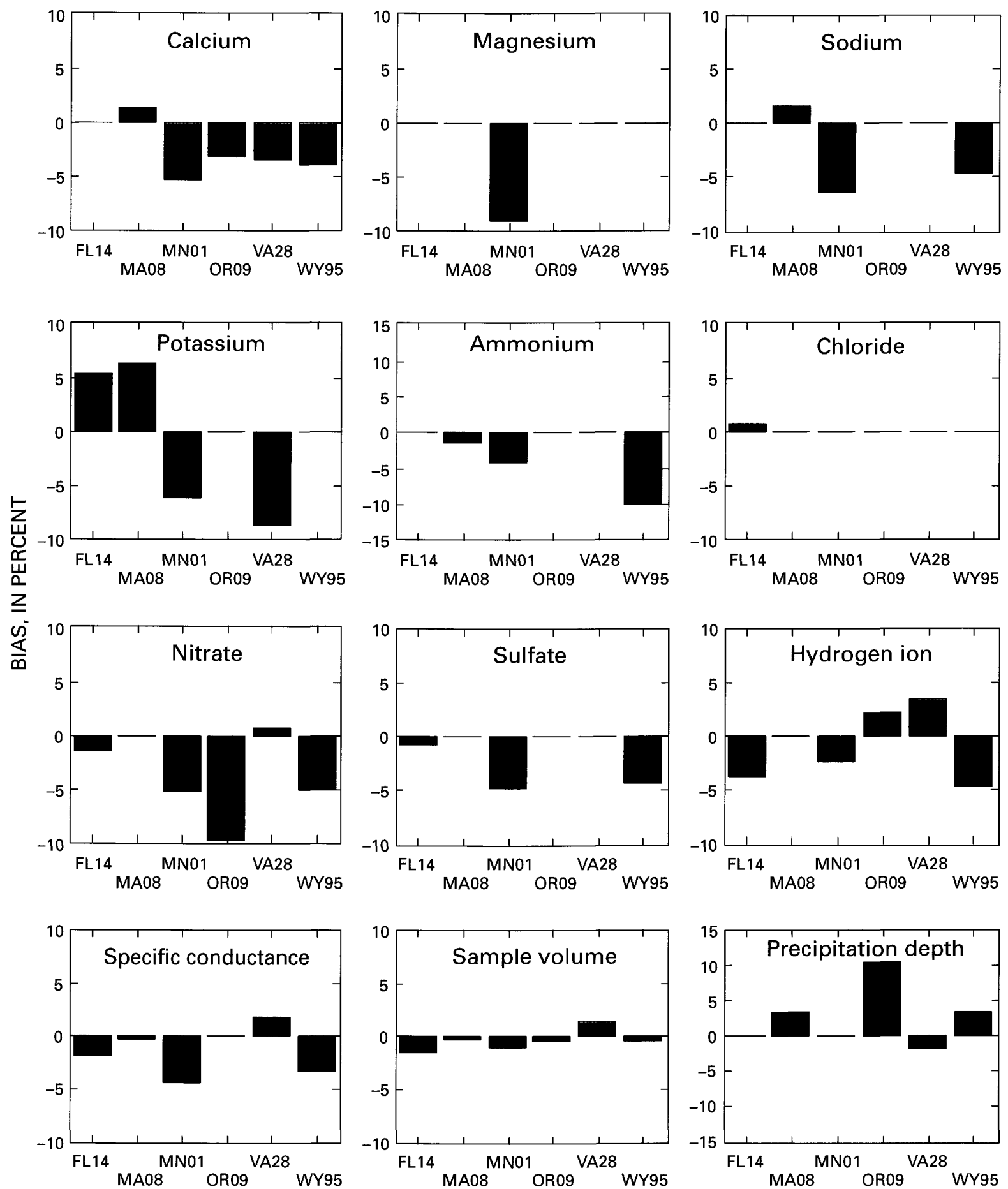

FOUR CHARACTER CODES OF THE ORIGINAL SAMPLING SITES*

\section{EXPLANATION}

* Data are from the original $\left(C_{1}\right)$ and collocated $\left(C_{2}\right)$ sites. For clarity, only the 4-digit code of the original sampling sites is displayed. Bias, or median relative error, in percent $=\left(\left(C_{1}-C_{2}\right) /\left(C_{1}+C_{2}\right) / 2\right) \times 100$

Figure 35. Bias for analyte concentration, deposition, and other physical parameters for weekly samples from collocated wet-dry precipitation collectors and precipitation depth from collocated rain gages during 1997 through 1999. All data are in percent. 


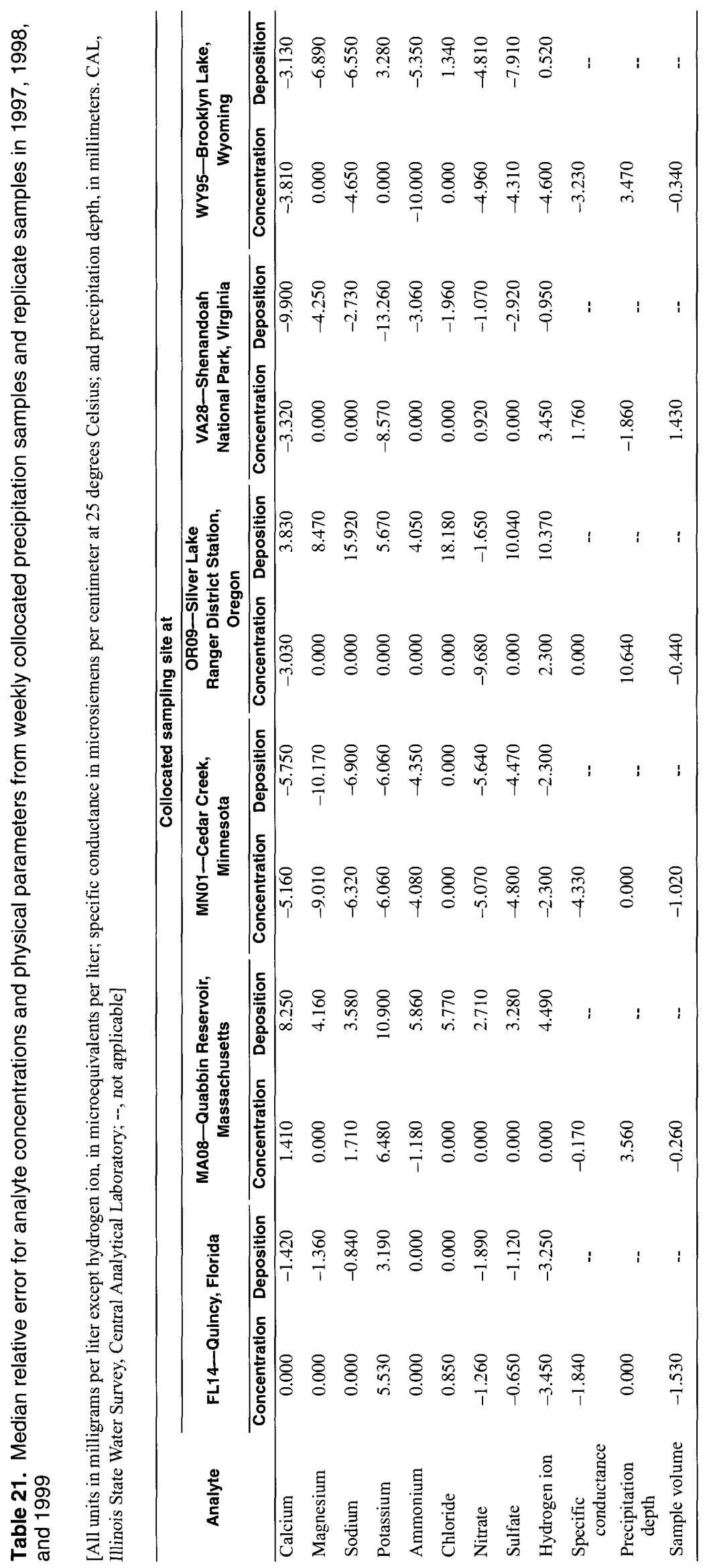


low bias associated with the Aerochem Metrics collectors during 1997-99. Bias in sample volume between collectors varied from -1.53 to +1.43 percent at collocated sites operated during 1997, 1998, and 1999.

This is a very small range for sample volume bias and was even better than the good results obtained during 1995-96 when the sample volume bias ranged from -1.5 to +3.5 percent.

For sample pairs with low concentrations of ionic constituents, the MAD (equation 8) can be very large, although the actual difference between the samples is small. For example, the sampling site with the least amount of precipitation, OR09/09OR, had the largest MAD values for sodium, magnesium, chloride, sulfate, and hydrogen ion when the MAD values were calculated using deposition amounts (figs. 36 and 37). The WY95/95WY site is located at an elevation of 3,213 meters above NAVD 88 and has the longest snow season of any of the sites in the 1997-99 collocated-sampler studies. It also is subject to frequent and severe windstorms throughout the year. Under these difficult sampling conditions, it is not surprising that the MAD values calculated using deposition amounts at the WY95/95WY site were often among the largest recorded among all of the sites. Blowing snow is extremely difficult to sample with the network's current sampling equipment.

The MA08/08MA site recorded the lowest MAD's for 5 of the 12 constituents-nitrate, sulfate, hydrogen ion, specific conductance, and sample volume. Frequently, the magnitudes of the MAD values (both in units of concentration and deposition) were inversely related to the magnitude of the median analyte concentration. For example, the FL14/14FL site had the highest levels of sodium and magnesium in its precipitation compared to the other sites, and had the lowest MAD's for magnesium and sodium. The MN01/01MN typically had high levels of calcium, potassium, and chloride in the precipitation recorded at this site; MN01/01MN had the lowest MAD for calcium, potassium, and chloride (figs. 36 and 37). VA28/28VA had the lowest concentration MAD for ammonium. In 1997-99, the MAD values for ionic constituent concentrations were the smallest for sulfate, less than 9 percent at each of the six sites.

Upon converting concentration amounts to deposition totals, the MAD's for most constituents increase (figs. 36 and 37). However, the MAD for calcium deposition at WY95/95WY, potassium deposition at OR09/09OR and WY95/95WY, ammonium deposition at FL14/14FL and WY95/95WY, chloride deposition at WY95/95WY, and nitrate deposition at VA28/28VA were all slightly lower than the corresponding MAD for the concentration value. For some analytes, the magnitude of the MAD for the deposition total is more than twice the magnitude of the MAD for concentration (for example, magnesium, sulfate, and hydrogen-ion deposition at OR09/09OR, ammonium and chloride deposition at VA28/28VA, and chloride deposition at MN01/01MN).

As in past years, the precision at the collocated sites for sample volume (measured from the Aerochem Metrics wet-deposition collectors) far exceeded the precision measured for precipitation depth (measured from the Belfort rain gages). The MAD's for sample volume were uniformly small (ranging from 2 to 5 percent). In contrast, the MAD's for precipitation depth ranged from less than 1 percent to over 10 percent during 1997-99, indicating there was some disparity in the performance of the Belfort rain gages at the various collocated sites. During 1995-96, sample volume MAD's also showed much better agreement than was observed for precipitation depth. The precipitation depth MAD ranged from 5 to 15 percent at the four collocated sites in 1996 and from 1 to 9 percent at the four 1995 collocated sites. In contrast, sample volume MAD's ranged from 2 to 5 percent during 1995-96 (Gordon, 1999). For researchers interested in using NADP/NTN deposition values, the variability introduced by precipitation depth measurements (as measured by the collocated program) is of concern. Graphical depictions of all MAD's for collocated sites are shown for concentration, for deposition, and for the physical measurements of sample volume and precipitation depth in figures 36 and 37.

As in past years, the MAD's were generally larger for cations than for anions. At many of the sites, cation concentrations were typically close to the minimum reporting limits, and larger variability is expected for concentrations approaching a minimum reporting limit because laboratory error usually increases as analyte concentration decreases. Estimates of network precision covering several years of collocated sampling are given in Nilles and others (1993). The MAD's for cations exceeded 7 percent at most sites in 1997-99 and occasionally exceeded 20 percent. The MAD's for anions ranged from 2 percent to 25 percent, 

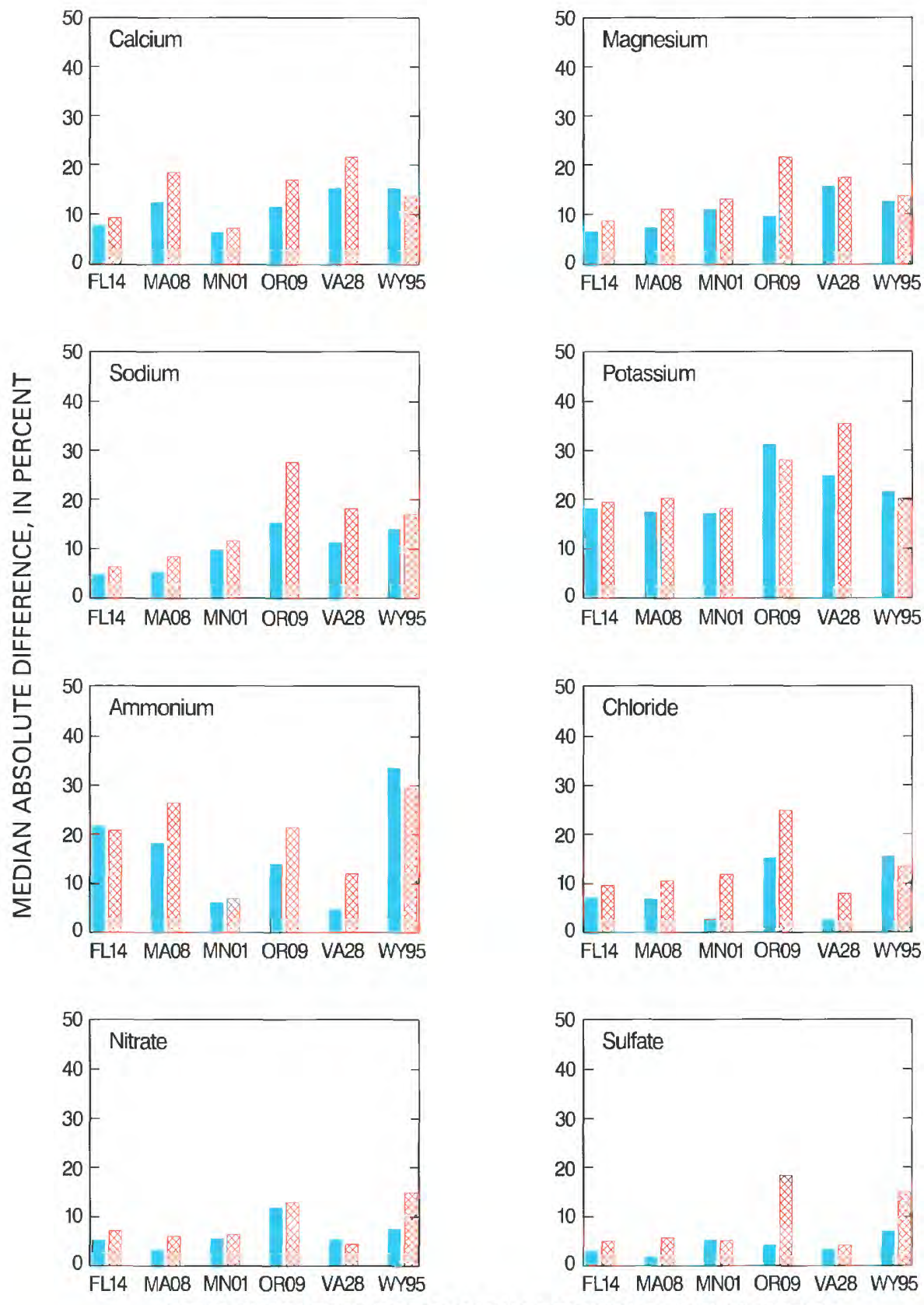

FOUR-CHARACTER CODES OF THE ORIGINAL SAMPLING SITES*

\section{EXPLANATION}

Error calculated from original and collocated concentration values

Error calculated from original and collocated deposition amounts

FL14* Data are from the original and collocated sites. For example, data from original site FL14 and collocated site 14FL is represented by the fourcharacter code of the original sampling site, FL14. For clarity, only the four-character codes of the original sample sites are displayed

Figure 36. Median absolute difference for analyte concentration and deposition for weekly samples from collocated precipitation collectors during 1997 through 1999 for selected analytes. All data are in percent. 

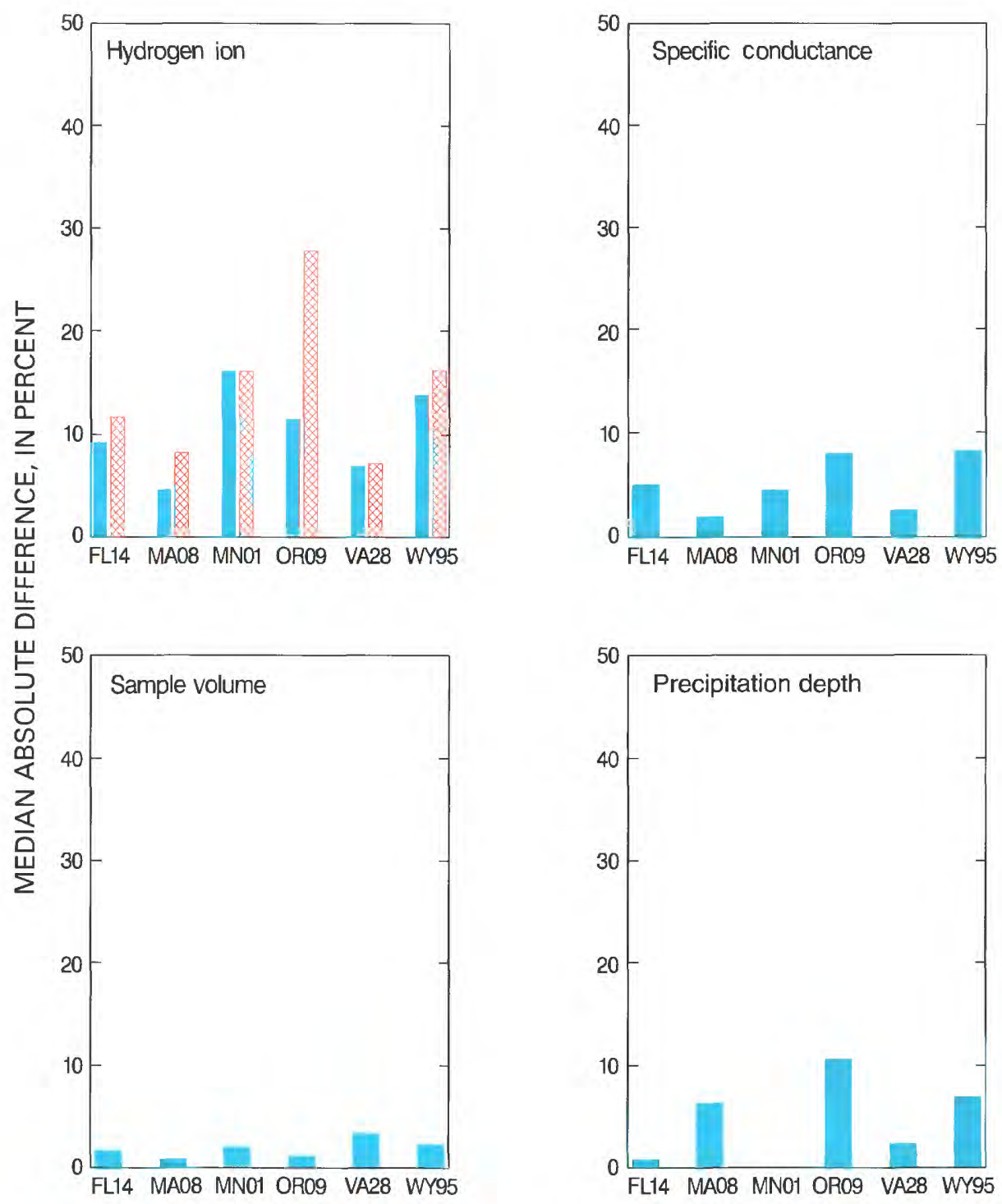

FOUR-CHARACTER CODES OF THE ORIGINAL SAMPLING SITES*

\section{EXPLANATION}

Error calculated from original and collocated concentration values

Error calculated from original and collocated deposition amounts

FL14* Data are from the original and collocated sites. For example, data from original site FL14 and collocated site 14FL is represented by the fourcharacter code of the original sampling site, FL14. For clarity, only the four-character codes of the original sample sites are displayed

Figure 37. Median absolute difference for hydrogen ion, specific conductance, sample volume, and precipitation depth for weekly samples from collocated precipitation collectors and precipitation depth from collocated rain gages during 1997 through 1999. 
reflecting the wide range in anion concentration values found at the 1997-99 collocated sites. Sites with the highest analyte concentration levels (fig. 33) -nitrate at MN01/01MN, MA08/08MA, and VA28/28VA and sulfate at FL14/14FL, MA08/08MA, MN01/01MN, and VA28/28VA - had correspondencing analyte MAD values of 5 percent or less. At the OR09/09OR and WY95/95WY sites where the sulfate and nitrate concentrations were much lower than for the other 1997-99 collocated sites (fig. 33), the MAD's were predictably much higher and generally exceeded 10 percent.

Consistent with the results from previous years of this study, the precision for hydrogen-ion concentration and deposition varied in absolute and in relative terms among the sites, depending upon the acidity of the precipitation at a given collocated site. The sites with the lowest median hydrogen-ion concentrations (fig. 34), MN01/01MN, OR09/09OR, and WY95/95WY, had much higher MAD's associated with their paired-sample analyses than MA08/08MA, VA28/28VA, or FL14/14FL, the sites with the highest median hydrogen-ion concentrations during 1997-99. The MAD's for hydrogen-ion concentration were less than 10 percent at MA08/08MA, VA28/28VA, and FL14/14FL (fig. 37), and these same sites had median hydrogen-ion concentrations of $18 \mu \mathrm{eq} / \mathrm{L}$ or greater (fig. 34). For MN01/01MN, OR09/09OR, and WY95/95WY, sites with median hydrogen-ion concentrations less than $10 \mu \mathrm{eq} / \mathrm{L}$ (fig. 34), the MAD's exceeded 12 percent (fig. 37).

\section{COMPARISON OF LABORATORY AND NETWORK ERROR}

Collocated analyte precision estimates in table 21 can be compared to analytical precision estimates calculated in the same manner from sample pairs submitted during 1997 through 1999 to the CAL as part of the interlaboratory-comparison program. The interlaboratory-comparison program is described in a preceding section of this report. Aliquots of natural, weekly, wet-deposition samples with volumes greater than $750 \mathrm{~mL}$ are used in the USGS interlaboratory-comparison program as well as synthetic precipitation samples. A comparison of the laboratory random error to the overall network error estimated from the collocated-sampler program indirectly provides a method to apportion the relative amount of error attributable to laboratory operations. Laboratory random error, as calculated from replicate samples submitted to the CAL for analysis in the interlaboratory-comparison program, typically accounted for less than one-sixth of the overall collocated-sampling error, although the fraction of sampling error attributable to laboratory random error varies with site and with analyte. During 1997-99, the CAL had a median relative error of $0.000 \mathrm{mg} / \mathrm{L}$ for magnesium, potassium, ammonium, chloride, nitrate, and sulfate. Using this direct comparison of collocated and interlaboratory data, one could argue that laboratory analysis made only a minor contribution to overall variability for these constituents during 1997-99 compared to variability introduced during sample collection, field processing, and shipping and handling of the samples. The comparison of laboratory random error to sampling error from specific NADP/NTN sites has limitations because sampling error is sitespecific for some analytes such as hydrogen ion. Specific partitioning of error at a given site would only be meaningful, for example, if the laboratory error term was calculated from a number of replicate samples collected at sites with similar hydrogen-ion concentration values.

\section{SUMMARY}

During 1997 through 1999, the U.S. Geological Survey used five programs to provide external qualityassurance monitoring for the National Atmospheric Deposition Program/National Trends Network (NADP/NTN). An intersite-comparison program was used to estimate the accuracy of onsite $\mathrm{pH}$ and specificconductance determinations. A blind-audit program was used to evaluate the effects of routine sample handling, processing, and shipping of wet-deposition samples on the bias and precision of NADP/NTN wetdeposition data. A field-audit program assessed the effects of field exposure of sample-collection equipment, handling, and processing on precipitation chemistry. An interlaboratory-comparison program assessed the bias and precision of analytical results determined by separate laboratories routinely measuring wet deposition. A collocated-sampler program was used to determine the overall bias and precision of NADP/NTN wetdeposition data at selected sites in the network. 


\section{Intersite-Comparison Program}

The intersite-comparison studies were held on a semiannual basis during 1997 through 1999. In intersite-comparison studies 39 through 43, the percentage of site operators responding on time that met the $\mathrm{pH}$ accuracy goals ranged from 83.7 percent to 90.9 percent. In these same five intersite-comparison studies, 92.4 to 99.4 percent of the site operators met the accuracy goals for specific conductance. The solutions used in the intersite-comparison program simulate natural rainwater with a wide range of hydrogen-ion concentration similar to that found in natural precipitation samples in the United States. In 1997-99, intersite samples were prepared with median $\mathrm{pH}$ values ranging from 4.12 to 4.80 .

\section{Blind-Audit Program}

The Wilcoxon signed-rank test was used to determine if significant differences existed between the analyte concentrations measured in paired blindaudit samples. One portion of the blind-audit sample was subject to all of the normal onsite handling and processing steps to which a regular weekly precipitation sample is subject. The other portion of the blindaudit sample was subject to minimal handling and served as the control portion. At a significance level of $\alpha=0.05$, the Wilcoxon signed-rank test indicated that bias was found only for potassium during 1997 through 1999. However, a paired t-test indicated a slight positive bias for calcium and chloride and a slight negative bias for hydrogen ion during 1997 through 1999 (table 6). The negative bias for ammonium and sodium found in 1995-96, reflecting the tendency for the portion of the blind-audit sample exposed to all of the handling and processing steps to actually have a lower concentration of these analytes than the minimally handled control portion of the blind-audit sample, has been eliminated since the November 1997 change in sample filters used at the CAL.

During 1997-99, the median (50th percentile) relative percent differences for all analytes were within a narrow range of -4.50 to +4.35 percent, indicating minor positive or negative bias. These results contrast with results from 1995-96, when ammonium had a median relative bias of -9.72 percent in 1995 and -12.50 percent in 1996 and the median relative percent bias for sodium was -7.99 percent in 1995 and -7.00 percent in 1996. Chloride also showed a high median relative percent bias of -7.04 percent in 1996.

During 1997-99, the median absolute differences in percent did not exceed 8 percent for any of the analytes. This is in contrast with the results from 1995-96 when, in addition to negative biases for ammonium and sodium, the median absolute difference values for ammonium and sodium ranged from 11.13 to 14.29 percent. During 1997 through 1999 , the median absolute difference was 3.33 percent for sodium and 4.41 percent for ammonium.

An equal number of samples containing 250 , 500 , or $1,000 \mathrm{~mL}$ of solution were included in each quarterly blind-audit mailing throughout 1997-99. Kruskal-Wallis results indicate a significant $(\alpha=0.05)$ relation between paired blind-audit sample differences in units of concentration and sample volume for magnesium, chloride, sulfate, and hydrogen ion during 1997-99. Before the January 1994 protocol change, at least 5 of the 10 measured analytes displayed a statistically significant relation between paired blind-audit differences in units of concentration and sample volume every year.

In 1997, sodium was reported at concentration levels that exceeded the method reporting limit in every field-exposed bucket portion of the Ultrapure deionized-water blind-audit sample and in 14 of 16 minimally handled bottle portions of the Ultrapure blind-audit samples. Following the change in the brand of filters used to process samples at the CAL in December 1997, the detection of sodium in Ultrapure samples dropped markedly: only 8 of 14 and 5 of 16 field-exposed bucket portions and 2 of 14 and 3 of 16 minimally handled bottle portions of Ultrapure samples had sodium levels exceeding the method reporting limit in 1998 and 1999, respectively. Chloride and nitrate also were commonly detected in the both the field-exposed and minimally handled Ultrapure samples used in the blind-audit program in 1997 but were detected less frequently in 1998 and 1999.

\section{Field-Audit Program}

The Wilcoxon signed-rank test was used to determine if significant differences existed between the analyte concentrations measured in paired fieldaudit samples. At a significance level of $\alpha=0.05$, the Wilcoxon signed-rank test indicated no significant 
difference for any of the analytes during I997-99 for either the "wet" or "dry" coded samples. However, a paired t-test indicated a significant difference for all of the analytes except sulfate and specific conductance for the "wet" coded samples and all but sodium and specific conductance for the "dry" coded samples during 1997 through 1999.

During I997-99, the median (50th percentile) relative percent difference for all analytes was within a range of -5.36 to +5.56 percent, indicating minor positive or negative bias. This result is a slightly larger range than the median relative percent bias for all analytes in the blind-audit program $(-4.50$ to +4.35$)$. During 1997-99, the median absolute differences in percent did not exceed 9 percent for any of the analytes. This result is similar to the result in the blindaudit program, which did not exceed 8 percent for any of its analytes during the same time period.

An equal number of samples containing 250, 500 , or $\mathrm{I}, 000 \mathrm{~mL}$ of solution were included in each quarterly field audit mailing throughout I997-99. Kruskal-Wallis results indicate a significant $(\alpha=0.05)$ relation between paired field-audit sample differences in units of concentration and sample volume for magnesium, chloride, and sulfate during I997-99. In I997, sodium was reported at concentration levels that exceeded the minimum reporting limit in all II of I I field-exposed bucket portions and minimally handled bottle portions of the Ultrapure field-audit samples. In I998, sodium detection decreased to 14 of 22 fieldexposed bucket portions and 2 of 22 minimally handled bottle portions of the Ultrapure field-audit samples. In I999, sodium detection again decreased to I 2 of 24 field-exposed bucket portions of the Ultrapure field-audit samples and no sodium was detected (out of 24) in the minimally handled bottle portions of the Ultrapure field-audit samples.

\section{Interlaboratory-Comparison Program}

In 1997, results of the Friedman test indicated significant $(\alpha=0.05)$ differences in all analyte measurements except for potassium among the five laboratories participating in the interlaboratory-comparison program. The multiple-comparison test, however, showed differences in the results for the various laboratories for all analytes, including potassium. In 1998 and 1999, both the Friedman and the multiplecomparison test showed significant differences in all analyte measurements. Cation precision estimates at the 50th percentile exhibited less variability than anions, $\mathrm{pH}$ (expressed as hydrogen-ion concentration), and specific conductance. Precision estimates at the 90th percentile exhibited some variability among the laboratories for cations, anions, $\mathrm{pH}$, and specific conductance. All participating laboratories had median absolute differences that were less than or equal to $0.005 \mathrm{mg} / \mathrm{L}$ for cations, less than or equal to $0.050 \mathrm{mg} / \mathrm{L}$ for anions.

In order to detect possible low-level sample contamination resulting from laboratory analyses, six Ultrapure deionized-water samples were included among the samples submitted to the participating laboratories during I997 through I999, as part of the interlaboratory-comparison program. For the purpose of comparing deionized water analyses in an equitable manner, reporting limits for all seven laboratories were standardized: if a minimum reporting limit was lower than the reporting limit used by the CAL for a certain analysis, the higher reporting limit used by the CAL was substituted. The CAL, SA, ADORC, and NILU did not detect concentrations greater than the "standardized" reporting limits for the Ultrapure samples for any analytes. The ESE reported seven concentrations greater than the "standardized" reporting limit for ammonium, one for sodium, and one for potassium during 1997 through 1999. The MOE reported one concentration for calcium and three concentrations for sodium and potassium greater than the "standardized" reporting limit. The MSC only reported one concentration greater than the "standardized" reporting limit for potassium.

\section{Collocated-Sampler Program}

A collocated-sampler program was used to estimate the overall bias and precision of chemical measurements of wet-deposition data collected for the NADP/NTN. Bias was not a major component of collocated results; bias in collocated measurements typically accounted for less than 20 percent of the overall error in collocated measurements. The estimates of precision include all variability in the datacollection system, from the point of sample collection through storage in the NADP/NTN database.

Weekly wet-deposition samples and precipitation measurements from collocated NADP/NTN sites were compared. Estimates of precision were calculated in terms of median relative error and median absolute difference for concentration and deposition of ionic constituents of wet deposition. The median relative 
error for sulfate and nitrate concentrations and for collected sample volumes was typically less than the median relative error calculated for the other analytes examined. Median relative error typically was greater for cations, with median relative error exceeding 7 percent at most sites. As in previous years, the precision for hydrogen-ion concentration and deposition varied greatly in absolute and relative terms among the sites, depending upon the acidity of the precipitation at a given collocated site. By comparing results from the interlaboratory-comparison program (replicate natural samples analyzed at the CAL) with results from the blind-audit and collocated-sampler programs, laboratory error was estimated in 1997-99 to account typically for less than one-sixth of the overall sampling error.

\section{REFERENCES CITED}

Berthouex, P.M., and Brown, L.C., 1995, Statistics for environmental engineers: Boca Raton, Fla., CRC Press, Inc., $336 \mathrm{p}$.

Bigelow, D.S., 1984, Instruction manual-NADP/NTN site selection and installation: National Atmospheric Deposition Program, Fort Collins, Natural Resources Ecology Laboratory, Colorado State University, 23 p.

Bigelow, D.S., and Dossett, S.R., 1988, Instruction manual-NADP/NTN site operation: National Atmospheric Deposition Program/National Trends Network, Fort Collins, Colorado State University, Natural Resources Ecology Laboratory, 114 p.

Bigelow, D.S., Sisterson, D.L., and Schroder, L.J., 1989, An interpretation of differences between field and laboratory $\mathrm{pH}$ values reported by the National Atmospheric Deposition Program/National Trends Network Monitoring Program: Environmental Science and Technology, v. 23, p. 881-887.

Chambers, J.M., Cleveland, W.S., Kleiner, B., and Tukey, P.A., 1983, Graphical methods for data analysis: Boston, Duxbury Press, p. 20-24.

Cleveland, W.S, 1985, The elements of graphing data: Monterey, Calif., Wadsworth, 323 p.

Conover, W.J., 1980, Practical nonparametric statistics ( $2 \mathrm{~d}$ ed.): New York, John Wiley, 493 p.

Dean, J.A., ed., 1979, Lange's handbook of chemistry (12th ed.): New York, McGraw-Hill, 644 p.

Gordon, J.D., 1999, External quality-assurance results for the National Atmospheric Deposition Program/National Trends Network, 1995-96: U.S. Geological Survey Water-Resources Investigations Report 99-4072, 69 p.
Gordon, J.D., Morden-Moore, A.L., Schroder, L.J., and Bowersox, V.C., 1995, The stability of hydrogen ion and specific conductance in filtered wet-deposition samples stored at ambient temperatures: Water, Air, and Soil Pollution, v. 83, p. 299-313.

Gordon, J.D., Nilles, M.A., Polacsek, D.K., and Ratcliff, M.E., 1997, External quality-assurance results for the National Atmospheric Deposition Program/National Trends Network during 1994: U.S. Geological Survey Water-Resources Investigations Report 97-4201, 42 p.

Gordon, J.D., Willoughby, T.C., and Schroder, L.J., 1991, Summary of the National Atmospheric Deposition Program/National Trends Network intersitecomparison program, November 1978-November 1989: U.S. Geological Survey Water-Resources Investigations Report 91-4061, 26 p.

Helsel, D.R., and Hirsch, R.M., 1992, Statistical methods in water resources: New York, Elsevier Science Publishing Company, Inc., 522 p.

Hem, J.D., 1985, Study and interpretation of the chemical characteristics of natural water ( $3 \mathrm{~d}$ ed.): U.S. Geological Survey Water-Supply Paper 2252, p. 62-63.

Hollander, Myles, and Wolfe, D.A, 1999, Nonparametric statistical methods ( $2 \mathrm{~d}$ ed.): New York, John Wiley and Sons, $787 \mathrm{p}$.

Huntsberger, D.V., and Billingsley, P., 1981, Elements of statistical inference (5th ed.): Boston, Allyn and Bacon, Inc., 503 p.

Iman, R.L., and Conover, W.J., 1983, A modern approach to statistics: New York, John Wiley, 496 p.

James, Kenni, 1996, 1995 Quality assurance report, NADP/NTN deposition monitoring, laboratory operations, Central Analytical Laboratory, January 1995 through December 1995: National Atmospheric Deposition Program/National Trends Network Coordination Office, Fort Collins, Colorado State University, Natural Resources Ecology Laboratory, 86 p.

Kanji, H.K., 1993, 100 Statistical tests: Newbury Park, Calif., Sage Publications Inc., 216 p.

Lynch, J.A., Bowersox, V.C., and Grimm, J.W., 1996, Trends in precipitation chemistry in the United States, 1983-94-An analysis of the effects in 1995 of Phase I of the Clean Air Act Amendments of 1990, Title IV: U.S. Geological Survey Open-File Report 96-0346, $100 \mathrm{p}$.

Miller, J.N., 1991, Statistical methods for analytical chemistry, part 2, Calibration and regression methods-A review: Analyst, January 1991, v. 116, p. 3-14.

National Atmospheric Deposition Program, 2000, National Atmospheric Deposition Program 1999 Wet Deposition, NADP Data Report 2000-02: Champaign, Illinois State Water Survey, 15 p. 
National lnstitute of Standards and Technology, 1991, Certificate for Standard Reference Material 2694A: Boulder, Colo., 1 p.

Nilles, M.A., 2001, Changes in the chemistry of precipitation in the United States, 1981-1998: Water, Air and Soil Pollution, v. 130, no. 1, p. 409-414.

Nilles, M.A., Gordon, J.D., and Schroder, L.J., 1993, Precision of wet atmospheric deposition data from National Atmospheric Deposition Program/National Trends Network sites determined with collocated samplers: Atmospheric Environment, v. 28, no. 6, p. 1121-1128.

Nilles, M.A., Gordon, J.D., Schroder, L.J., and Paulin, C.E., 1995, External quality-assurance results for the National Atmospheric Deposition Program/National Trends Network during 1991: U.S. Geological Survey Water-Resources Investigations Report 93-4208, 36 p.

Nilles, M.A., See, R.B., Willoughby, T.C., and Gordon, J.D., 1991, Variability in wet atmospheric deposition data determined with collocated samplers: U.S. Geological Survey Water-Resources Investigations Report 91-4143, 30 p.

Ostle, Bernard, and Malone, L.C., 1988, Statistics in research—Basic concepts and techniques for research workers, 4th ed.: Ames, Iowa State University Press, Iowa, p. 106.
Peden, M.E., 1986, Development of standard methods for collection and analysis of precipitation, in Methods for collection and analysis of precipitation: Champaign, Illinois State Water Survey Contract Report 381, p. 19-21.

Peden, M.E., and Skowron, L.M., 1978, Ionic stability of precipitation samples: Atmospheric Environment, v. 12 , p. $2343-2349$.

Robertson, J.K., and Wilson, J.W., 1985, Design of the National Trends Network for monitoring the chemistry of atmospheric precipitation: U.S. Geological Survey Circular 964, $46 \mathrm{p}$.

SAS Institute Inc., 1989, SAS STAT users guide, version 6, 4th ed., v.1: Cary, N.C., SAS Institute Inc.

See, R.B., Willoughby, T.C., Brooks, M.H., and Gordon, J.D., 1990, Programs and analytical methods for the U.S. Geological Survey Acid Rain Quality-Assurance project: U.S. Geological Survey Water-Resources Investigations Report 90-4029, 57 p.

See, R.B., Willoughby, T.C., and Schroder, L.J., 1989, External quality-assurance results for the National Atmospheric Deposition Program/National Trends Network during 1987: U.S. Geological Survey WaterResources Investigations Report 89-4015, 16 p.

Taylor, J.K., 1987, Quality assurance of chemical measurements, 2d. ed.: Chelsea, Mich., Lewis Publishers, Inc., $328 \mathrm{p}$. 





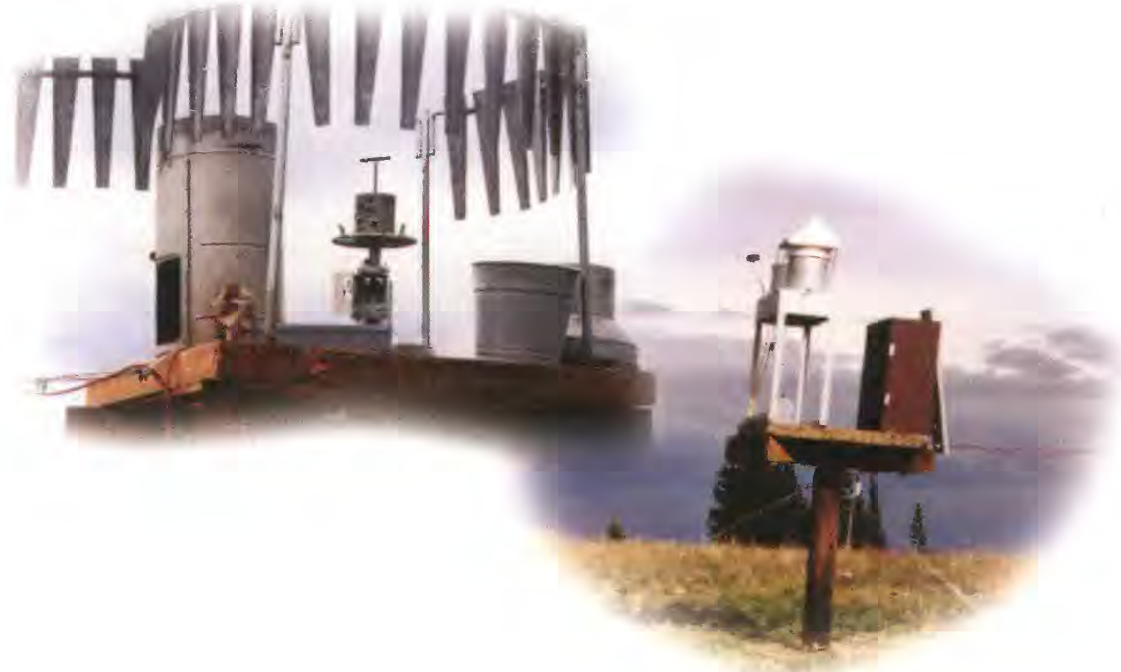

